Supporting Information

\title{
Exploiting Alkylquinone Tautomerization: Amine Benzylation
}

\author{
Luis M. Mori-Quiroz and Michael D. Clift \\ Department of Chemistry, The University of Kansas, Lawrence, KS, 66047 \\ *email: mclift@ku.edu
}

Table of Contents

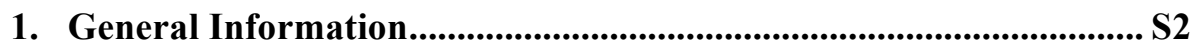

2. Summary of Optimization Experiments.......................................... S3

3. Preparation of Alkylquinones...........................................................S4

4. Amine Benzylation with Alkylquinones ............................................ S6

5. NOESY studies for compounds 33a and 33b .................................... S15

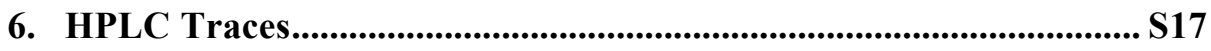

7. Copies of ${ }^{1} \mathrm{H},{ }^{13} \mathrm{C}$ and ${ }^{19} \mathrm{~F}$ NMR Spectra .......................................... S21 


\section{General Information}

Reactions were run in oven-dried vials under nitrogen atmosphere, stirred magnetically and heated in an aluminum block. iso-Propanol was distilled from magnesium sulfate, sparged with nitrogen triethylamine was distilled from calcium hydride. All other amines were used as received. Toluene, acetonitrile, THF, dichloromethane were purified by passing the solvent through activated alumina using a solvent purification system. Purification of products was carried out by flash chromatography using silica gel (230-400 Mesh, Grade 60). Reactions were monitored by Thin Layer Chromatography (TLC) (silica gel $60 \mathrm{~F}_{254}$ ) and visualized using UV or $\mathrm{KMnO}_{4}$, PMA and CAM stain solutions. ${ }^{1} \mathrm{H}$ NMR, ${ }^{13} \mathrm{C}$ NMR and ${ }^{19} \mathrm{~F}$ NMR spectra were recorded at room temperature in a Bruker 400 or Bruker 500 instruments. Chemical shifts are reported in $\mathrm{ppm}$ and referenced with respect to residual protic chloroform at $7.26 \mathrm{ppm}\left({ }^{1} \mathrm{H} \mathrm{NMR}\right)$ and the carbon resonances of the solvent at $77 \mathrm{ppm}\left({ }^{13} \mathrm{C} \mathrm{NMR}\right)$. The following abbreviations were used to refer to multiplicities: $\mathrm{s}=\operatorname{singlet}, \mathrm{d}=$ doublet, $\mathrm{t}=$ triplet, $\mathrm{q}=$ quadruplet, $\mathrm{m}=$ multiplet. Infrared spectra was recorded using a Shimadzu FTIR-8400S spectrometer. Mass spectra were obtained on a Micromass LCT Premier quadrupole and time-of-flight tandem mass analyzer. 


\section{Summary of Optimization Experiments}

Procedure: A dry 7-mL vial was charged with stirbar, 2,6-dimethyl-1,4-benzoquinone (40.9 mg, 0.3 mmol), capped and evacuated / backfilled with nitrogen (3 cycles). Then, iso-propanol (1 mL), dibenzylamine (59.2 $\mathrm{mg}, 0.3 \mathrm{mmol}, 1$ equiv), and triethylamine ( $42 \mu \mathrm{L}, 0.3 \mathrm{mmol}, 1$ equiv) were added sequentially by syringe. The nitrogen line was removed and the septa sealed with grease. The mixture was stirred in an aluminum block at the appropriate temperature (see table $\mathbf{S 1}$ for specific temperatures and reaction times). The reaction was then cooled at room temperature and 1,3,5-trimethoxybenzene ( $50.5 \mathrm{mg}, 0.3 \mathrm{mmol}, 1$ equiv) was added, followed by $\sim 1 \mathrm{~mL}$ of EtOAc to dissolve it. The crude reaction mixture was then concentrated in vacuo and analyzed by ${ }^{1} \mathrm{H}$ NMR (Table S1).

Table S1. Summary of optimization experiments.

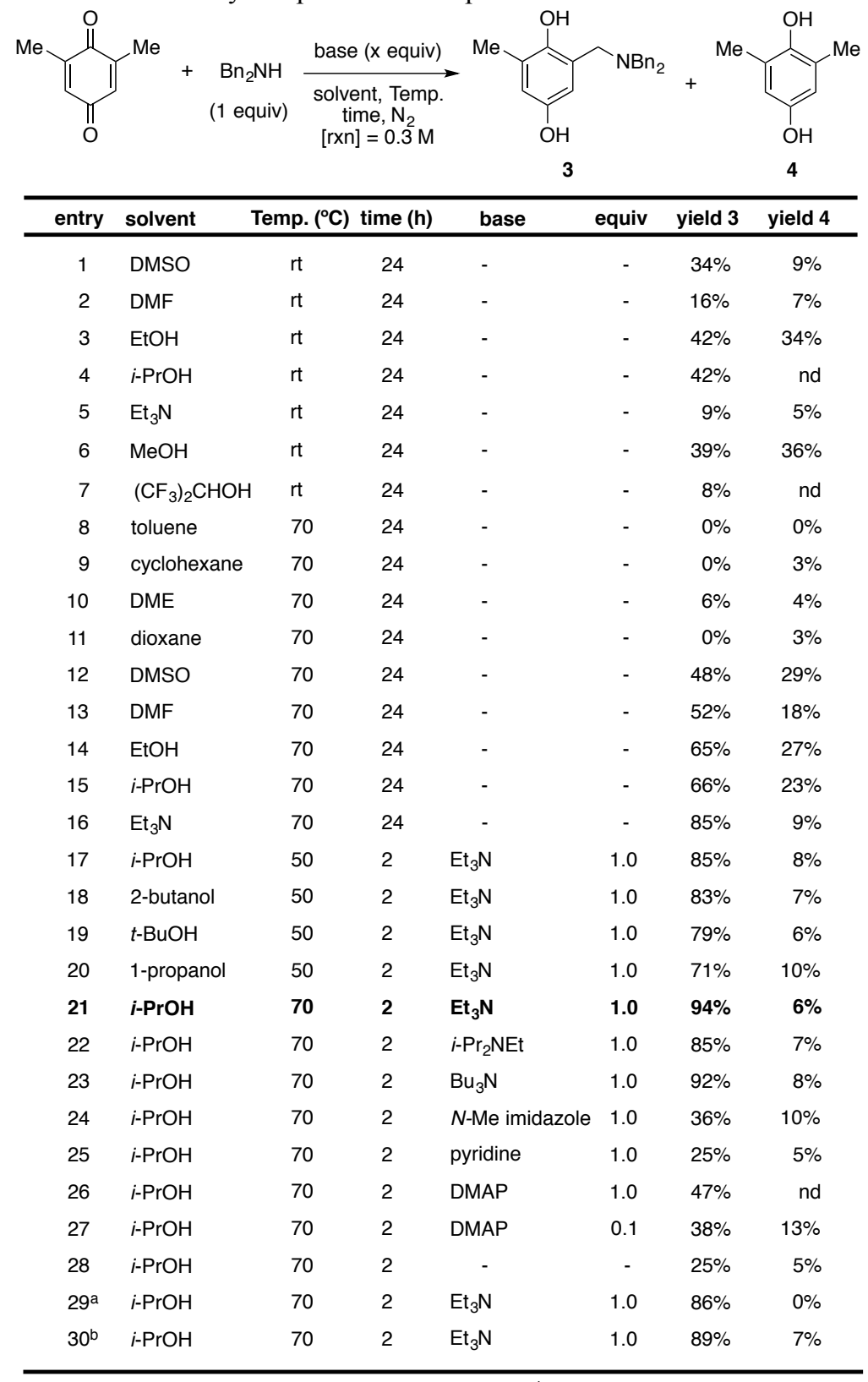

nd $=$ not determined. ${ }^{a}$ Run under oxygen atmosphere. ${ }^{b}$ Run in the dark. 


\section{Preparation of Alkylquinones}

\section{Preparation of 4-chloro-5-methyl-1,2-benzoquinone (S1)}<smiles>O=C1C=CC(=O)C(Cl)=C1</smiles>

S1

Following a reported procedure, ${ }^{1} 3$-methyl-4-chlorophenol $(2 \mathrm{~g}, 14 \mathrm{mmol})$ was dissolved in acetone $(60 \mathrm{~mL})$ and water $(150 \mathrm{~mL})$ was added. On a separate flask Fremy's salt ( $8.3 \mathrm{~g}, 30.86 \mathrm{mmol}, 2.2$ equiv) was dissolved in a solution of $0.17 \mathrm{M}$ $\mathrm{KH}_{2} \mathrm{PO}_{4}$ in water $(100 \mathrm{~mL})$. The solution of Fremy's salt was then added to the solution of phenol with vigorous stirring, under air. The mixture was stirred at room temperature for $1.5 \mathrm{~h}$. The reaction mixture was extracted with $\mathrm{CH}_{2} \mathrm{Cl}_{2}(3 \times 100 \mathrm{~mL})$. Combined organic extracts were washed with brine, dried over $\mathrm{MgSO}_{4}$, filtered and concentrated. Column chromatography (8\% EtOAc in hexanes) afforded $148 \mathrm{mg}(11 \%)$ of $\mathbf{S 1}$ as a yellow solid and a mixture of S1, its regioisomeric orthoquinone and unreacted 3-methyl-4chlorophenol. The reaction was not optimized. m.p. $=104-105^{\circ} \mathrm{C} ;{ }^{1} \mathrm{H} \mathrm{NMR}\left(400 \mathrm{MHz}, \mathrm{CDCl}_{3}\right) \delta 6.98$ (s, $1 \mathrm{H}), 6.74(\mathrm{q}, J=1.6 \mathrm{~Hz}, 1 \mathrm{H}), 2.07(\mathrm{~d}, J=1.6 \mathrm{~Hz}, 3 \mathrm{H}) ;{ }^{13} \mathrm{C} \mathrm{NMR}\left(101 \mathrm{MHz}, \mathrm{CDCl}_{3}\right) \delta 185.4,179.5$, 146.6, 143.9, 133.6, 132.7, 15.6; FTIR $\left(\mathrm{cm}^{-1}\right) 3049,1643,1593,1566,1377,1236,1119,981$; HRMS (ESI) $m / z 157.0045\left[(\mathrm{M}+\mathrm{Na})^{+}\right.$; calcd for $\left.\mathrm{C}_{7} \mathrm{H}_{6} \mathrm{ClO}_{2}^{+}, 157.0051\right]$.

\section{Preparation of tert-butyl (2-(4'-methyl-2,5-dioxo-2,5-dihydro-[1,1'-biphenyl]-4-} yl)ethyl)carbamate (S2)

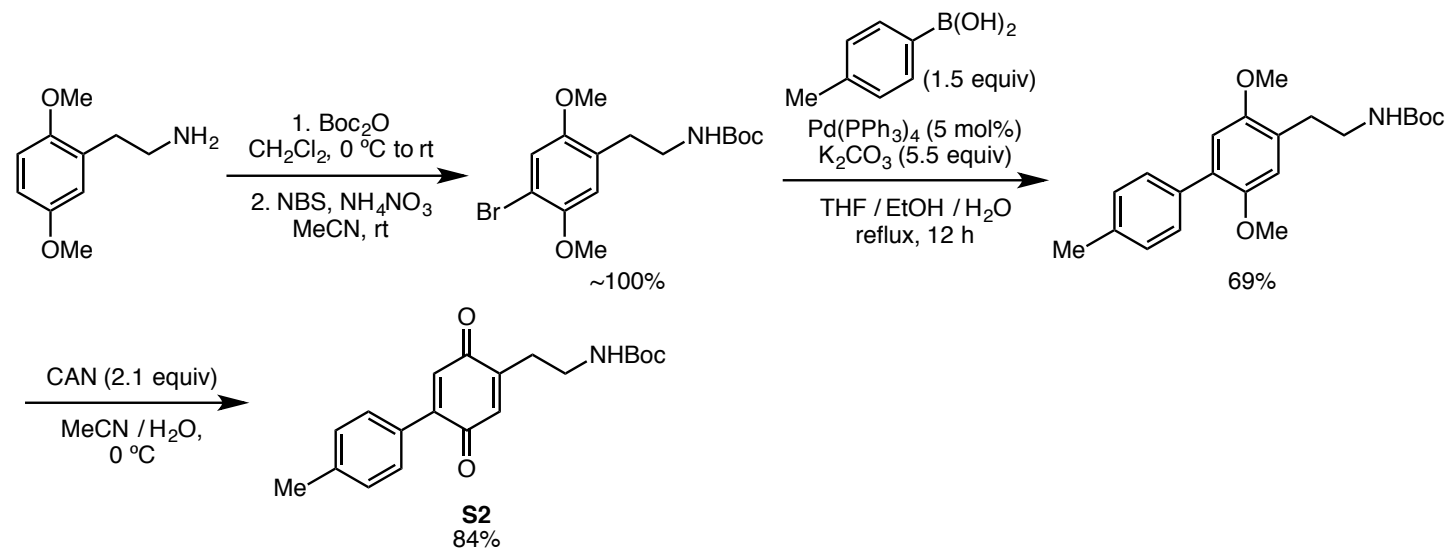

A dry round bottom flask was charged with 2-(2,5-dimethoxyphenyl)ethan-1-amine (3 $\mathrm{g}, 16.56 \mathrm{mmol})$ and dry $\mathrm{CH}_{2} \mathrm{Cl}_{2}(50 \mathrm{~mL})$. The solution was cooled at $0{ }^{\circ} \mathrm{C}$ and $\mathrm{Boc}_{2} \mathrm{O}(3.8 \mathrm{~g}, 17.39 \mathrm{mmol}, 1.05$ equiv) was added in one portion (gas evolution). The reaction was slowly warmed to room temperature as the ice bath expired. The reaction was concentrated in vacuo and the crude product (white solid) was used in the next step without further purification. $\mathrm{Mp}=84-86{ }^{\circ} \mathrm{C} ;{ }^{1} \mathrm{H}$ NMR $\left(500 \mathrm{MHz}, \mathrm{CDCl}_{3}\right) \delta 6.78(\mathrm{~d}, J$ $=9.4 \mathrm{~Hz}, 1 \mathrm{H}), 6.73(\mathrm{dd}, J=7.2,2.8 \mathrm{~Hz}, 2 \mathrm{H}), 4.66(\mathrm{~s}, 1 \mathrm{H}), 3.78(\mathrm{~s}, 3 \mathrm{H}), 3.76(\mathrm{~s}, 3 \mathrm{H}), 3.35(\mathrm{q}, J=6.5$ $\mathrm{Hz}, 2 \mathrm{H}), 2.78(\mathrm{t}, J=6.8 \mathrm{~Hz}, 2 \mathrm{H}), 1.43(\mathrm{~s}, 9 \mathrm{H}) ;{ }^{13} \mathrm{C} \mathrm{NMR}\left(126 \mathrm{MHz}, \mathrm{CDCl}_{3}\right) \delta 156.0,153.5,151.8$, 128.6, 116.8, 111.8, 111.2, 78.96, 55.8, 55.7, 40.6, 30.9, 28.4. FTIR $\left(\mathrm{cm}^{-1}\right) 3358,2976,1712,1225$, 1045.

Following a reported procedure, ${ }^{2}$ tert-butyl (2,5-dimethoxyphenethyl)carbamate prepared in the previous step was partially dissolved in dry $\mathrm{MeCN}(25 \mathrm{~mL})$ and a mixture of NBS $(3.1 \mathrm{~g}, 17.39 \mathrm{mmol}$, 1.05 equiv) and ammonium nitrate $(130 \mathrm{mg}, 1.66 \mathrm{mmol}, 0.1$ equiv) was added. The resulting orange solution was vigorously stirred for $2.5 \mathrm{~h}$ at room temperature. A yellow precipitate formed over time. Water $(25 \mathrm{~mL})$ was added, and the mixture was extracted with $\mathrm{Et}_{2} \mathrm{O}(3 \times 100 \mathrm{~mL})$. The combined organic extracts were washed with brine, dried over magnesium sulfate and concentrated in vacuo to give $6.15 \mathrm{~g}(\sim 100 \%)$ of tert-butyl (4-bromo-2,5-dimethoxyphenethyl)carbamate as a tan solid. The product was used in the next step without further purification. A small amount was purified by column chromatography $\left(15 \%\right.$ EtOAc in hexanes) for characterization purposes. White solid; $\mathrm{mp}=95-96{ }^{\circ} \mathrm{C}$;

\footnotetext{
${ }^{1}$ Goldstein, S. L.; McNelis, E. J. Org. Chem. 1984, 49, 1613-1620.

${ }^{2}$ Yang, J.; Knueppel, D.; Cheng, B.; Mans, D.; Martin, S. F. Org. Lett. 2015, 17, 114-117.
} 
${ }^{1} \mathrm{H}$ NMR $\left(500 \mathrm{MHz}, \mathrm{CDCl}_{3}\right) \delta 7.03(\mathrm{~s}, 1 \mathrm{H}), 6.73(\mathrm{~s}, 1 \mathrm{H}), 4.61(\mathrm{~s}, 1 \mathrm{H}), 3.84(\mathrm{~s}, 3 \mathrm{H}), 3.77(\mathrm{~s}, 3 \mathrm{H}), 3.32$ $(\mathrm{q}, J=6.5 \mathrm{~Hz}, 2 \mathrm{H}), 2.77(\mathrm{t}, J=6.9 \mathrm{~Hz}, 2 \mathrm{H}), 1.42(\mathrm{~s}, 9 \mathrm{H}) ;{ }^{13} \mathrm{C} \mathrm{NMR}\left(126 \mathrm{MHz}, \mathrm{CDCl}_{3}\right) \delta 155.9,151.9$, 149.9, 127.6, 115.8, 114.9, 109.1, 79.12, 56.9, 56.1, 40.3, 30.8, 28.4; FTIR (cm $\left.{ }^{-1}\right) 3356,2974,1712$, 1495, 1215, 1170, 1037. HRMS (ESI) $\mathrm{m} / z$ 382.0639 [(M+Na) ${ }^{+}$; calcd for $\left.\mathrm{C}_{15} \mathrm{H}_{22} \mathrm{BrNO}_{4} \mathrm{Na}^{+}, 382.0630\right]$.

A Schlenk flask was charged with stirbar, tert-butyl (4-bromo-2,5-dimethoxyphenethyl)carbamate (1.8 g, $5 \mathrm{mmol})$, $p$-tolylboronic acid (1.02 g, $7.49 \mathrm{mmol}, 1.5 \mathrm{equiv})$, potassium carbonate $(3.8 \mathrm{~g}, 27.5 \mathrm{mmol}$, 5.5 equiv) and $\mathrm{Pd}\left(\mathrm{PPh}_{3}\right)_{4}(290 \mathrm{mg}, 0.25 \mathrm{mmol}, 0.05$ equiv $)$. The flask was sealed with rubber septa and evacuated / backfilled with nitrogen $(3$ cycles $)$, then THF $(17 \mathrm{~mL})$, water $(4 \mathrm{~mL})$ and ethanol $(4 \mathrm{~mL})$ were added by syringe. The mixture was degassed by freeze-pump-thaw (3 cycles). The reaction was heated to reflux for $14 \mathrm{~h}$ with vigorous stirring. The reaction was cooled at room temperature, water $(10 \mathrm{~mL})$ was added and the mixture was extracted with EtOAc $(3 \times 30 \mathrm{~mL})$. Combined organic extracts were washed with brine, dried over magnesium sulfate and concentrated in vacuo. Column chromatography $\left(\mathrm{CH}_{2} \mathrm{Cl}_{2}\right)$ and recrystallization from hot hexanes afforded $1.28 \mathrm{~g}(69 \%)$ of tert-butyl (2-(4'-methyl-2,5-dioxo-2,5-dihydro-[1,1'-biphenyl]-4-yl)ethyl)carbamate as an off-white solid. m.p. = 114-116 ${ }^{\circ} \mathrm{C} ;{ }^{1} \mathrm{H}$ NMR $\left(400 \mathrm{MHz}, \mathrm{CDCl}_{3}\right) \delta 7.42(\mathrm{~d}, J=7.9 \mathrm{~Hz}, 2 \mathrm{H}), 7.22(\mathrm{~d}, J=7.9 \mathrm{~Hz}, 2 \mathrm{H}), 6.82(\mathrm{~s}$, $1 \mathrm{H}), 6.79(\mathrm{~s}, 1 \mathrm{H}), 4.72(\mathrm{~s}, 1 \mathrm{H}) 3.81(\mathrm{~s}, 3 \mathrm{H}), 3.74(\mathrm{~s}, 3 \mathrm{H}), 3.38(\mathrm{~m}, 2 \mathrm{H}), 2.84(\mathrm{t}, J=6.9 \mathrm{~Hz}, 2 \mathrm{H}), 2.39$ (s, $3 \mathrm{H}), 1.44(\mathrm{~s}, 9 \mathrm{H}) ;{ }^{13} \mathrm{C}$ NMR $\left(101 \mathrm{MHz} \mathrm{CDCl}_{3}\right) \delta 156.0,151.7,150.3,136.7,135.6,129.3,128.8$, 127.1, 114.8, 113.4, 79.00, 56.4, 56.0, 40.8, 30.8, 28.5, 21.2; FTIR ( $\left.\mathrm{cm}^{-1}\right)$ 3363, 2974, 1685, 1529, 1496, 1206, 1150, 1045; HRMS (ESI) $m / z$ 394.2006 [(M+Na) ${ }^{+}$; calcd for $\left.\mathrm{C}_{22} \mathrm{H}_{29} \mathrm{NO}_{4} \mathrm{Na}^{+}, 394.1994\right]$.

A $50 \mathrm{~mL}$ round bottom flask was charged with tert-butyl (2-(4'-methyl-2,5-dioxo-2,5-dihydro-[1,1'biphenyl]-4-yl)ethyl)carbamate (450 mg, $1.21 \mathrm{mmol}), \mathrm{MeCN}(12 \mathrm{~mL})$ and $\mathrm{CH}_{2} \mathrm{Cl}_{2}(2 \mathrm{~mL})$. The solution was cooled at $0{ }^{\circ} \mathrm{C}$ and water $(3 \mathrm{~mL})$ was added, followed by ceric ammonium nitrate $(1.39 \mathrm{~g}$, $2.54 \mathrm{mmol}, 2.1$ equiv) in small portions over $30 \mathrm{~s}$ with vigorous stirring. After 30 minutes water (10 $\mathrm{mL})$ was added, and the mixture was extracted with $\mathrm{CH}_{2} \mathrm{Cl}_{2}(3 \times 20 \mathrm{~mL})$. Combined organic extracts were washed with water $(2 \times 20 \mathrm{~mL})$, brine, dried over magnesium sulfate and concentrated in vacuo to afford $343 \mathrm{mg}(84 \%)$ of $\mathbf{S 2}$ as a yellow solid. m.p. $=144{ }^{\circ} \mathrm{C}$ (decomp.); ${ }^{1} \mathrm{H}$ NMR $\left(500 \mathrm{MHz}, \mathrm{CDCl}_{3}\right)$ $\delta 7.38(\mathrm{~d}, J=8.3 \mathrm{~Hz}, 2 \mathrm{H}), 7.24(\mathrm{~m}, J=8.3 \mathrm{~Hz}, 2 \mathrm{H}), 6.85(\mathrm{~s}, 1 \mathrm{H}), 6.66(\mathrm{~s}, 1 \mathrm{H}), 4.69(\mathrm{~d}, J=6.4 \mathrm{~Hz}$, $1 \mathrm{H}), 3.36(\mathrm{q}, J=6.5 \mathrm{~Hz}, 2 \mathrm{H}), 2.68-2.61(\mathrm{~m}, 2 \mathrm{H}), 2.39(\mathrm{~s}, 3 \mathrm{H}), 1.39(\mathrm{~s}, 9 \mathrm{H}) ;{ }^{13} \mathrm{C} \mathrm{NMR}(126 \mathrm{MHz}$, $\left.\mathrm{CDCl}_{3}\right) \delta 187.6,187.0,155.9,146.1,145.6,140.4,134.1,132.3,129.7,129.24,129.17,79.5,39.0$, 30.4, 28.3, 21.4; FTIR ( $\left.\mathrm{cm}^{-1}\right)$ 3341, 2974, 1699, 1653, 1242, 1207, 1157; HRMS (ESI) $\mathrm{m} / z$ 364.1524 $\left[(\mathrm{M}+\mathrm{Na})^{+}\right.$; calcd for $\left.\mathrm{C}_{20} \mathrm{H}_{23} \mathrm{NO}_{4} \mathrm{Na}^{+}, 364.1525\right]$.

\section{Preparation of 4'-fluoro-4,6-dimethyl-[1,1'-biphenyl]-2,5-dione (S3)}

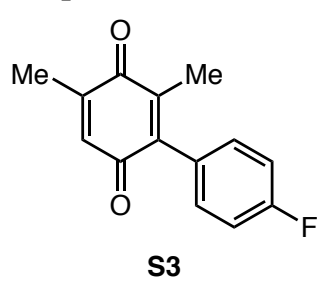

Following a reported procedure, ${ }^{3}$ a $100 \mathrm{~mL}$ round bottom flask was charged with stirbar, 2,6-dimethyl-1,4-benzoquinone (800 $\mathrm{mg}, 5.88 \mathrm{mmol}$ ), trifluorotoluene $(30 \mathrm{~mL})$ and 4-fluorophenylboronic acid $(1.23 \mathrm{~g}, 8.81 \mathrm{mmol}$, 1.5 equiv). A solution of $\mathrm{AgNO}_{3}(200 \mathrm{mg}, 1.175 \mathrm{mmol}, 0.2$ equiv) in water (18 mL) was added, followed by $\mathrm{K}_{2} \mathrm{~S}_{2} \mathrm{O}_{8}$ (4.77 g, $17.63 \mathrm{mmol}, 3$ equiv). The mixture was vigorously stirred under air at room temperature. After $14 \mathrm{~h}$ the mixture was extracted with $\mathrm{CH}_{2} \mathrm{Cl}_{2}(3 \times 20 \mathrm{~mL})$. Combined organic extracts were dried over magnesium sulfate and concentrated in vacuo. Column chromatography (50-70\% $\mathrm{CH}_{2} \mathrm{Cl}_{2}$ in hexanes) afforded $804 \mathrm{mg}(59 \%)$ of $\mathbf{S 3}$ as an orange oil. ${ }^{1} \mathrm{H}$ NMR $\left(500 \mathrm{MHz}, \mathrm{CDCl}_{3}\right) \delta 7.12(\mathrm{~m}, 4 \mathrm{H}), 6.66(\mathrm{q}, J=1.6 \mathrm{~Hz}, 1 \mathrm{H}), 2.11(\mathrm{~d}, J=1.7 \mathrm{~Hz}, 3 \mathrm{H}), 1.97(\mathrm{~s}, 3 \mathrm{H}) ;{ }^{13} \mathrm{C}$ NMR $\left(126 \mathrm{MHz}, \mathrm{CDCl}_{3}\right) \delta 188.4,186.6,162.7(\mathrm{~d}, J=248.4 \mathrm{~Hz}), 145.7,142.7,141.8,133.1,131.5(\mathrm{~d}$, $J=8.2 \mathrm{~Hz}), 128.7(\mathrm{~d}, J=3.4 \mathrm{~Hz}), 115.3(\mathrm{~d}, J=21.6 \mathrm{~Hz}), 16.0,14.2 ;{ }^{19} \mathrm{~F} \mathrm{NMR}\left(376 \mathrm{MHz}, \mathrm{CDCl}_{3}\right) \delta-$ 112.7; FTIR $\left(\mathrm{cm}^{-1}\right)$ 2960, 1651, 1508, 1225, 1159.

${ }^{3}$ Fujiwara, Y.; Domingo, V.; Seiple, I. B.; Gianatassio, R.; Del Bel, M.; Baran, P. S. J. Am. Chem. Soc. 2011, 133, 3292-3295. 
Preparation of 2-(4-methoxyphenyl)-3-methylnaphthalene-1,4-dione (S4)

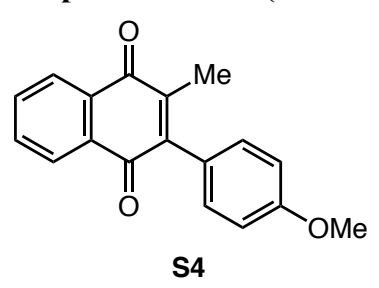

Following a reported procedure, ${ }^{3}$ a $100 \mathrm{~mL}$ round bottom flask was charged with stirbar, 2-methyl-1,4-naphthoquinone (1 g, $5.81 \mathrm{mmol})$, trifluorotoluene $(30 \mathrm{~mL})$ and 4-methoxyphenylboronic acid $(1.32 \mathrm{~g}, 8.71$ mmol, 1.5 equiv). A solution of $\mathrm{AgNO}_{3}(197 \mathrm{mg}, 1.16 \mathrm{mmol}, 0.2$ equiv) in water $(18 \mathrm{~mL})$ was added, followed by $\mathrm{K}_{2} \mathrm{~S}_{2} \mathrm{O}_{8}(4.71 \mathrm{~g}, 17.4 \mathrm{mmol}, 3$ equiv). The mixture was vigorously stirred under air at room temperature. After $17 \mathrm{~h}$ the mixture was extracted with $\mathrm{CH}_{2} \mathrm{Cl}_{2}(3 \times 20 \mathrm{~mL})$. Combined organic extracts were dried over magnesium sulfate and concentrated in vacuo. Column chromatography (10\% EtOAc in hexanes) afforded $252 \mathrm{mg}(16 \%)$ of $\mathbf{S 4}$ as a yellow solid. m.p. $=172-173{ }^{\circ} \mathrm{C} ;{ }^{1} \mathrm{H}$ NMR $\left(400 \mathrm{MHz}, \mathrm{CDCl}_{3}\right) \delta 8.12(\mathrm{~m}, 2 \mathrm{H}), 7.72(\mathrm{~m}, 2 \mathrm{H}), 7.18(\mathrm{~d}, J=8.6$ $\mathrm{Hz}, 2 \mathrm{H}), 6.99(\mathrm{~d}, J=8.4 \mathrm{~Hz}, 2 \mathrm{H}), 3.86(\mathrm{~s}, 3 \mathrm{H}), 2.12(\mathrm{~s}, 3 \mathrm{H}) ;{ }^{13} \mathrm{C} \mathrm{NMR}\left(101 \mathrm{MHz}, \mathrm{CDCl}_{3}\right) \delta 185.81$, $184.4,159.8,145.8,143.8,133.5,133.4,132.2,132.1,131.0,126.6,126.1,125.7,113.6,55.3,14.8$; FTIR $\left(\mathrm{cm}^{-1}\right) 3030,2835,1661,1606,1595,1505,1290,1250,1179$.

\section{Amine Benzylation with Alkylquinones - General Procedure A}

A dry $7-\mathrm{mL}$ vial was charged with stirbar, quinone $(0.3 \mathrm{mmol})$, capped and evacuated / backfilled with nitrogen (3 cycles). Then iso-propanol $(1 \mathrm{~mL})$, secondary (or primary) amine (1 equiv), and triethylamine ( $42 \mu \mathrm{L}, 1$ equiv) were added sequentially by syringe. The nitrogen line was removed and the septa sealed with grease. The mixture was heated in an aluminum block at $70{ }^{\circ} \mathrm{C}$ for $2 \mathrm{~h}$ (unles otherwise noticed). The reaction was then cooled at room temperature, concentrated in vacuo, and the product purified by column chromatography.

\section{2-((dibenzylamino)methyl)-6-methylbenzene-1,4-diol (3)}

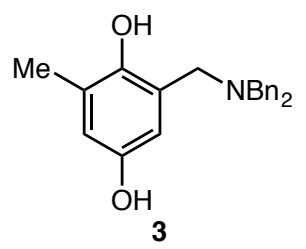

According to general procedure A, 2,6-dimethyl-1,4-benzoquinone $(40.9 \mathrm{mg}$, $0.3 \mathrm{mmol})$, dibenzylamine $(59.2 \mathrm{mg}, 0.3 \mathrm{mmol}, 1$ equiv) and triethylamine (42 $\mu \mathrm{L}, 0.3 \mathrm{mmol}, 1$ equiv) in iso-propanol $(1 \mathrm{~mL})$ for $2 \mathrm{~h}$ at $70{ }^{\circ} \mathrm{C}$, afforded after silica gel chromatography (3-4\% $\mathrm{MeCN}$ in $\left.\mathrm{CH}_{2} \mathrm{Cl}_{2}\right), 92.4 \mathrm{mg}(92 \%)$ of 3 as an oil. ${ }^{1} \mathrm{H}$ NMR $\left(400 \mathrm{MHz}, \mathrm{CDCl}_{3}\right) \delta 7.38-7.27(\mathrm{~m}, 10 \mathrm{H}), 6.55(\mathrm{~d}, J=3.0$ $\mathrm{Hz}, 1 \mathrm{H}), 6.40(\mathrm{~d}, J=2.9 \mathrm{~Hz}, 1 \mathrm{H}), 3.64(\mathrm{~s}, 2 \mathrm{H}), 3.59(\mathrm{~s}, 4 \mathrm{H}), 2.22(\mathrm{~s}, 3 \mathrm{H}) ;{ }^{13} \mathrm{C}$ NMR $\left(101 \mathrm{MHz}, \mathrm{CDCl}_{3}\right) \delta 149.1,147.8,136.7,129.5,128.6,127.6,125.8$, 121.9, 116.7, 113.2, 57.8, 56.8, 16.0; FTIR $\left(\mathrm{cm}^{-1}\right)$ 3024, 2818, 1209, 1150, 773; HRMS (ESI) $\mathrm{m} / \mathrm{z}$ $334.1797\left[(\mathrm{M}+\mathrm{H})^{+}\right.$; calcd for $\left.\mathrm{C}_{22} \mathrm{H}_{24} \mathrm{NO}_{2}{ }^{+}, 334.1807\right]$.

\section{2-((allyl(phenethyl)amino)methyl)-6-methylbenzene-1,4-diol (5)}<smiles>C=CCN(CCc1ccccc1)Cc1cc(O)cc(C)c1O</smiles>

5 According to general procedure A, 2,6-dimethyl-1,4-benzoquinone (40.9 $\mathrm{mg}, 0.3 \mathrm{mmol}), N$-allyl-2-phenylethylamine ( $48.4 \mathrm{mg}, 0.3 \mathrm{mmol}, 1$ equiv) and triethylamine ( $42 \mu \mathrm{L}, 0.3 \mathrm{mmol}, 1$ equiv) in iso-propanol $(1 \mathrm{~mL})$ for $2 \mathrm{~h}$ at $70{ }^{\circ} \mathrm{C}$, afforded after silica gel chromatography $(25 \%$ EtOAc in hexanes), $79.5 \mathrm{mg}(89 \%)$ of 5 as a light yellow oil. ${ }^{1} \mathrm{H}$ NMR $\left(400 \mathrm{MHz}, \mathrm{CDCl}_{3}\right) \delta$ $7.30-7.24(\mathrm{~m}, 2 \mathrm{H}), 7.20(\mathrm{~m}, 1 \mathrm{H}), 7.12(\mathrm{~m}, 2 \mathrm{H}), 6.55(\mathrm{~d}, J=2.9 \mathrm{~Hz}, 1 \mathrm{H})$, $6.34(\mathrm{~d}, J=3.0 \mathrm{~Hz}, 1 \mathrm{H}), 5.89$ (ddt, $J=17.1,10.6,6.7 \mathrm{~Hz}, 1 \mathrm{H}), 5.27-5.18$

(m, 2H), 3.74 (s, 2H), $3.22(\mathrm{dt}, J=6.7,1.2 \mathrm{~Hz}, 2 \mathrm{H}), 2.88-2.77(\mathrm{~m}, 4 \mathrm{H}), 2.17$ (s, 3H); ${ }^{13} \mathrm{C}$ NMR $(101$ $\left.\mathrm{MHz}, \mathrm{CDCl}_{3}\right) \delta 149.7,147.6,139.4,133.3,128.58,128.48,126.3,125.9,121.8,119.5,116.6,112.8$, 57.3, 56.0, 54.6, 32.8, 15.8; FTIR $\left(\mathrm{cm}^{-1}\right) 3375,3026,2924,2830,1479,1229,912$; HRMS (ESI) $\mathrm{m} / \mathrm{z}$ $298.1786\left[(\mathrm{M}+\mathrm{H})^{+}\right.$; calcd for $\left.\mathrm{C}_{19} \mathrm{H}_{24} \mathrm{NO}_{2}{ }^{+}, 298.1802\right]$.

\section{2-((diallylamino)methyl)-6-methylbenzene-1,4-diol (6)}<smiles>C=CCN(CC=C)Cc1cc(O)cc(C)c1O</smiles>
According to general procedure A, 2,6-dimethyl-1,4-benzoquinone (40.9 $\mathrm{mg}, 0.3 \mathrm{mmol})$, diallylamine $(37 \mu \mathrm{L}, 0.3 \mathrm{mmol}, 1$ equiv) and triethylamine (42 $\mu \mathrm{L}, 0.3 \mathrm{mmol}, 1$ equiv) in iso-propanol $(1 \mathrm{~mL})$ for $2 \mathrm{~h}$ at $70{ }^{\circ} \mathrm{C}$, afforded after silica gel chromatography (21\% EtOAc in hexanes), $57.5 \mathrm{mg}$

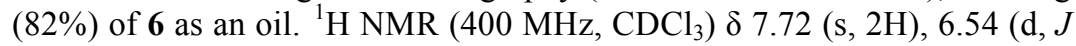
$=2.9 \mathrm{~Hz}, 1 \mathrm{H}), 6.33(\mathrm{~d}, J=2.9 \mathrm{~Hz}, 1 \mathrm{H}), 5.86(\mathrm{ddt}, J=17.0,10.4,6.7 \mathrm{~Hz}$, $2 \mathrm{H}), 5.26-5.15(\mathrm{~m}, 4 \mathrm{H}), 3.66(\mathrm{~s}, 2 \mathrm{H}), 3.14(\mathrm{~d}, J=6.7 \mathrm{~Hz}, 4 \mathrm{H}), 2.17(\mathrm{~s}$, $3 \mathrm{H}) ;{ }^{13} \mathrm{C}$ NMR $\left(101 \mathrm{MHz}, \mathrm{CDCl}_{3}\right) \delta 149.4,147.79,133.3,125.8,121.8,119.6,116.6,112.9,56.5$, 
55.7, 15.76; FTIR $\left(\mathrm{cm}^{-1}\right)$ 3356, 3070, 2972, 1240, 1200, 1140; HRMS (ESI) $\mathrm{m} / z 234.1492\left[(\mathrm{M}+\mathrm{H})^{+}\right.$; calcd for $\left.\mathrm{C}_{14} \mathrm{H}_{20} \mathrm{NO}_{2}^{+}, 234.1489\right]$.

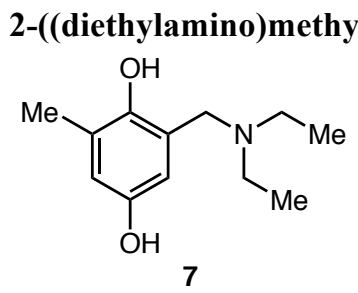

Compound 7 was prepared by a modification of general procedure A: According to general procedure A, 2,6-dimethyl-1,4-benzoquinone (40.9 $\mathrm{mg}, 0.3 \mathrm{mmol})$, diethylamine ( $31 \mu \mathrm{L}, 0.3 \mathrm{mmol}, 1$ equiv), triethylamine (42 $\mu \mathrm{L}, 0.3 \mathrm{mmol}, 1$ equiv) and iso-propanol $(1 \mathrm{~mL})$ were combined in a vial under nitrogen and the reaction was run for $24 \mathrm{~h}$ at room temperature. Concentration and silica gel chromatography (20\% EtOAc in hexanes) afforded $40.6 \mathrm{mg}(65 \%)$ of 7 as a light brown oil. ${ }^{1} \mathrm{H}$ NMR $(400 \mathrm{MHz}$, $\left.\mathrm{CDCl}_{3}\right) \delta 8.13(\mathrm{~s}, 2 \mathrm{H}), 6.54(\mathrm{~d}, J=3.0 \mathrm{~Hz}, 1 \mathrm{H}), 6.35(\mathrm{~d}, J=3.0 \mathrm{~Hz}, 1 \mathrm{H}), 3.65(\mathrm{~s}, 2 \mathrm{H}), 2.60(\mathrm{q}, J=7.2$ $\mathrm{Hz}, 4 \mathrm{H}), 2.17(\mathrm{~s}, 3 \mathrm{H}), 1.10(\mathrm{~d}, J=7.2 \mathrm{~Hz}, 4 \mathrm{H}) ;{ }^{13} \mathrm{C} \mathrm{NMR}\left(101 \mathrm{MHz}, \mathrm{CDCl}_{3}\right) \delta 149.6,147.8,125.8$, 122.0, 116.5, 112.7, 56.7, 46.2, 15.8, 11.1; FTIR $\left(\mathrm{cm}^{-1}\right) 3340,2968,1254,1192,1142$. HRMS (ESI) $m / z 210.1494\left[(\mathrm{M}+\mathrm{H})^{+} ;\right.$calcd for $\left.\mathrm{C}_{12} \mathrm{H}_{20} \mathrm{NO}_{2}^{+}, 210.1489\right]$.

\section{2-((allyl(methyl)amino)methyl)-6-methylbenzene-1,4-diol (8)}

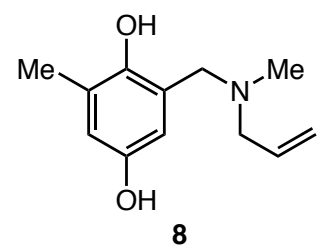

Compound $\mathbf{8}$ was prepared by a modification of general procedure A: According to general procedure A, 2,6-dimethyl-1,4-benzoquinone (40.9 mg, $0.3 \mathrm{mmol})$, methylallylamine $(29 \mu \mathrm{L}, 0.3 \mathrm{mmol}, 1$ equiv), triethylamine $(210$ $\mu \mathrm{L}, 0.3 \mathrm{mmol}, 5$ equiv), iso-propanol $(0.2 \mathrm{~mL})$ and Toluene $(0.8 \mathrm{~mL})$ were combined in a vial under nitrogen and the reaction was run for $24 \mathrm{~h}$ at room temperature. Concentration and silica gel chromatography $(10-30 \% \mathrm{MeCN}$ in $\left.\mathrm{CH}_{2} \mathrm{Cl}_{2}\right)$ afforded $36.5 \mathrm{mg}(59 \%)$ of $\mathbf{8}$ as a light red oil. ${ }^{1} \mathrm{H}$ NMR $(400 \mathrm{MHz}$, $\left.\mathrm{CDCl}_{3}\right) \delta 7.84(\mathrm{~s}, 2 \mathrm{H}), 6.55(\mathrm{~d}, J=2.9 \mathrm{~Hz}, 1 \mathrm{H}), 6.35(\mathrm{~d}, J=2.9 \mathrm{~Hz}, 1 \mathrm{H}), 5.91-5.81(\mathrm{~m}, 1 \mathrm{H}), 5.23-$ $5.20(\mathrm{~m}, 2 \mathrm{H}), 3.60(\mathrm{~s}, 2 \mathrm{H}), 3.10(\mathrm{~d}, J=6.6 \mathrm{~Hz}, 2 \mathrm{H}), 2.26(\mathrm{~s}, 3 \mathrm{H}), 2.17(\mathrm{~s}, 3 \mathrm{H}) ;{ }^{13} \mathrm{C}$ NMR $(101 \mathrm{MHz}$, $\left.\mathrm{CDCl}_{3}\right) \delta 149.4,147.8,133.5,125.8,121.7,119.3,116.7,112.8,60.2,59.6,41.0,15.8 ;$ FTIR $\left(\mathrm{cm}^{-1}\right)$ 3349, 3078, 2918, 1479, 1229, 995; HRMS (ESI) $\mathrm{m} / z$ 208.1338 [(M+H) ${ }^{+}$; calcd for $\mathrm{C}_{12} \mathrm{H}_{18} \mathrm{NO}_{2}^{+}$, 208.1338].

2-((cyclohexyl(methyl)amino)methyl)-6-methylbenzene-1,4-diol (9)

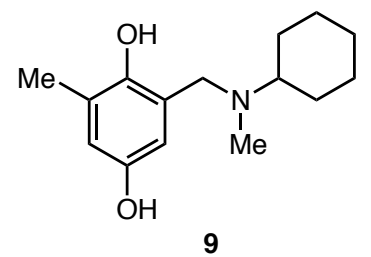

According to general procedure A, 2,6-dimethyl-1,4-benzoquinone (40.9 $\mathrm{mg}, 0.3 \mathrm{mmol}$ ), $N$-methylcyclohexylamine ( $39 \mu \mathrm{L}, 0.3 \mathrm{mmol}, 1$ equiv) and triethylamine ( $42 \mu \mathrm{L}, 0.3 \mathrm{mmol}, 1$ equiv) in iso-propanol $(1 \mathrm{~mL})$ for $2 \mathrm{~h}$ at $70{ }^{\circ} \mathrm{C}$, afforded after silica gel chromatography $(40 \%$ EtOAc in hexanes), $63.5 \mathrm{mg}(85 \%)$ of 9 as a tan solid. m.p. $=152-153{ }^{\circ} \mathrm{C}$ (decomp.); ${ }^{1} \mathrm{H}$ NMR (400 MHz, $\left.\mathrm{CDCl}_{3}\right) \delta 7.75$ (s, 2H), 6.53 (d, $J=3.0$ $\mathrm{Hz}, 1 \mathrm{H}), 6.33(\mathrm{~d}, J=3.0 \mathrm{~Hz}, 1 \mathrm{H}), 3.71(\mathrm{~s}, 2 \mathrm{H}), 2.54(\mathrm{tt}, J=11.3,3.4 \mathrm{~Hz}$, $1 \mathrm{H}), 2.24(\mathrm{~s}, 3 \mathrm{H}), 2.17(\mathrm{~s}, 3 \mathrm{H}), 1.89-1.79(\mathrm{~m}, 4 \mathrm{H}), 1.64(\mathrm{~m}, 1 \mathrm{H}), 1.39-1.17(\mathrm{~m}, 4 \mathrm{H}), 1.17-1.03(\mathrm{~m}$, $1 \mathrm{H}) ;{ }^{13} \mathrm{C}$ NMR $\left(101 \mathrm{MHz}, \mathrm{CDCl}_{3}\right) \delta 150.1,147.4,125.6,122.0,116.3,112.6,62.2,57.4,36.3,28.1$, 26.1, 25.7, 15.7; FTIR $\left(\mathrm{cm}^{-1}\right) 3335,2932,2856,1477,1217,910$; HRMS (ESI) $\mathrm{m} / z 250.1799$ [(M+H) ${ }^{+}$; calcd for $\left.\mathrm{C}_{15} \mathrm{H}_{24} \mathrm{NO}_{2}^{+}, 250.1807\right]$.

\section{2-((dicyclohexylamino)methyl)-6-methylbenzene-1,4-diol (10)}

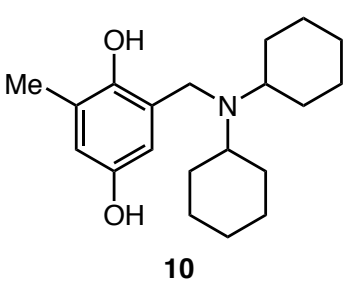

Compound $\mathbf{1 0}$ was prepared by a modification of general procedure A: According to general procedure A, 2,6-dimethyl-1,4-benzoquinone (40.9 $\mathrm{mg}, 0.3 \mathrm{mmol})$, dicyclohexylamine $(60 \mu \mathrm{L}, 0.3 \mathrm{mmol}, 1$ equiv), triethylamine $(210 \mu \mathrm{L}, 0.3 \mathrm{mmol}, 5$ equiv), iso-propanol $(0.2 \mathrm{~mL})$ and Toluene $(0.8 \mathrm{~mL})$ were combined in a vial under nitrogen and the reaction was run for $3 \mathrm{~h}$ at $70{ }^{\circ} \mathrm{C}$. Concentration and silica gel chromatography $\left(15 \%\right.$ and $20 \% \mathrm{MeCN}$ in $\left.\mathrm{CH}_{2} \mathrm{Cl}_{2}\right)$ afforded $71.6 \mathrm{mg}$ $(75 \%)$ of 10 as a tan solid. m.p. $=154-155{ }^{\circ} \mathrm{C} ;{ }^{1} \mathrm{H}$ NMR $(400 \mathrm{MHz}$,

$\left.\mathrm{CDCl}_{3}\right) \delta 6.51(\mathrm{~d}, J=2.9 \mathrm{~Hz}, 1 \mathrm{H}), 6.32(\mathrm{~d}, J=2.9 \mathrm{~Hz}, 1 \mathrm{H}), 3.84(\mathrm{~s}, 2 \mathrm{H}), 2.67(\mathrm{tt}, J=11.8,3.4 \mathrm{~Hz}$, 2H), $2.16(\mathrm{~s}, 3 \mathrm{H}), 1.87-1.61(\mathrm{~m}, 8 \mathrm{H}), 1.60(\mathrm{~d}, J=12.3 \mathrm{~Hz}, 2 \mathrm{H}), 1.36$ (qd, $J=12.2,3.1 \mathrm{~Hz}, 4 \mathrm{H}), 1.21$ $(\mathrm{m}, 4 \mathrm{H}), 1.12-0.98(\mathrm{~m}, 2 \mathrm{H}) ;{ }^{13} \mathrm{C}$ NMR $\left(101 \mathrm{MHz}, \mathrm{CDCl}_{3}\right) \delta 150.3,147.3,125.5,123.0,116.0,112.4$, 
57.8, 49.6, 30.6, 26.0, 25.9, 15.7; FTIR $\left(\mathrm{cm}^{-1}\right)$ 3327, 2928, 2852, 1477, 1204, 1150; HRMS (ESI) $\mathrm{m} / \mathrm{z}$ $318.2447\left[(\mathrm{M}+\mathrm{H})^{+}\right.$; calcd for $\left.\mathrm{C}_{20} \mathrm{H}_{32} \mathrm{NO}_{2}^{+}, 318.2433\right]$.

\section{2-((diisopropylamino)methyl)-6-methylbenzene-1,4-diol (11)}<smiles>Cc1cc(O)cc(CN(C(C)C)C([O])[O-])c1O</smiles>

11

Compound $\mathbf{1 1}$ was prepared by a modification of general procedure A: According to general procedure A, 2,6-dimethyl-1,4-benzoquinone (40.9 $\mathrm{mg}, 0.3 \mathrm{mmol})$, diisopropylamine ( $42 \mu \mathrm{L}, 0.3 \mathrm{mmol}, 1$ equiv), triethylamine $(210 \mu \mathrm{L}, 0.3 \mathrm{mmol}, 5$ equiv), iso-propanol $(0.2 \mathrm{~mL})$ and Toluene $(0.8 \mathrm{~mL})$ were combined in a vial under nitrogen and the reaction was run for $3 \mathrm{~h}$ at $70{ }^{\circ} \mathrm{C}$. Concentration and silica gel chromatography $(20 \% \mathrm{MeCN}$ in $\left.\mathrm{CH}_{2} \mathrm{Cl}_{2}\right)$ afforded $44.6 \mathrm{mg}(63 \%)$ of $\mathbf{1 1}$ as a brown oil. ${ }^{1} \mathrm{H}$ NMR $(400 \mathrm{MHz}$, $\left.\mathrm{CDCl}_{3}\right) \delta 8.42(\mathrm{~s}, 2 \mathrm{H}), 6.52(\mathrm{~d}, J=2.8 \mathrm{~Hz}, 1 \mathrm{H}), 6.36(\mathrm{~d}, J=2.9 \mathrm{~Hz}, 1 \mathrm{H}), 3.74(\mathrm{~s}, 2 \mathrm{H}), 3.12(\mathrm{~h}, J=6.7$ $\mathrm{Hz}, 2 \mathrm{H}), 2.16(\mathrm{~s}, 3 \mathrm{H}), 1.10(\mathrm{~d}, J=6.7 \mathrm{~Hz}, 12 \mathrm{H}) ;{ }^{13} \mathrm{C}$ NMR $\left(101 \mathrm{MHz}, \mathrm{CDCl}_{3}\right) \delta 150.0,147.7,125.5$, 122.5, 116.1, 112.5, 48.5, 47.7, 19.7, 15.8; FTIR $\left(\mathrm{cm}^{-1}\right)$ 3323, 2931, 1477, 1197, 1146, 777; HRMS (ESI) $m / z 238.1812\left[(\mathrm{M}+\mathrm{H})^{+}\right.$; calcd for $\left.\mathrm{C}_{14} \mathrm{H}_{24} \mathrm{NO}_{2}{ }^{+}, 238.1807\right]$.

\section{2-methyl-6-(morpholinomethyl)benzene-1,4-diol (12)}<smiles>Cc1cc(O)cc(CN2CCOCC2)c1O</smiles>

12

According to general procedure A, 2,6-dimethyl-1,4-benzoquinone (40.9 $\mathrm{mg}, 0.3 \mathrm{mmol})$, morpholine $(26 \mu \mathrm{L}, 0.3 \mathrm{mmol}, 1$ equiv) and triethylamine (42 $\mu \mathrm{L}, 0.3 \mathrm{mmol}, 1$ equiv) in iso-propanol $(1 \mathrm{~mL})$ for $2 \mathrm{~h}$ at $70^{\circ} \mathrm{C}$, afforded after silica gel chromatography (50\% EtOAc in hexanes), $46.8 \mathrm{mg}(82 \%)$ of 12 as a light yellow oil. ${ }^{1} \mathrm{H}$ NMR $\left(400 \mathrm{MHz}, \mathrm{CDCl}_{3}\right) \delta 7.67(\mathrm{~s}, 2 \mathrm{H}), 6.55(\mathrm{~d}$, $J=3.0 \mathrm{~Hz}, 1 \mathrm{H}), 6.34(\mathrm{~d}, J=2.9 \mathrm{~Hz}, 1 \mathrm{H}), 3.75(\mathrm{t}, J=4.7 \mathrm{~Hz}, 4 \mathrm{H}), 3.60(\mathrm{~s}$, $2 \mathrm{H}), 2.55(\mathrm{~s}, 4 \mathrm{H}), 2.18(\mathrm{~s}, 3 \mathrm{H}) ;{ }^{13} \mathrm{C} \mathrm{NMR}\left(101 \mathrm{MHz}, \mathrm{CDCl}_{3}\right) \delta 149.1$, 147.9, 125.9, 120.6, 116.9, 113.0, 66.8, 61.8, 52.8, 15.8; FTIR $\left(\mathrm{cm}^{-1}\right)$ 3368, 2945, 1477, 1204, 1150; HRMS (ESI) $m / z 224.1290\left[(\mathrm{M}+\mathrm{H})^{+}\right.$; calcd for $\left.\mathrm{C}_{12} \mathrm{H}_{18} \mathrm{NO}_{3}{ }^{+}, 224.1281\right]$.

tert-butyl 4-(2,5-dihydroxy-3-methylbenzyl)piperazine-1-carboxylate (13)<smiles>Cc1cc(O)cc(CN2CCN(C(=O)OCc3ccccc3)CC2)c1O</smiles>

13

According to general procedure A, 2,6-dimethyl-1,4-benzoquinone (40.9 $\mathrm{mg}, 0.3 \mathrm{mmol}), N$-Bocpiperazine $(55.9 \mathrm{mg}, 0.3 \mathrm{mmol}, 1$ equiv) and triethylamine $(42 \mu \mathrm{L}, 0.3 \mathrm{mmol}, 1$ equiv) in iso-propanol $(1 \mathrm{~mL})$ for $2 \mathrm{~h}$ at $70{ }^{\circ} \mathrm{C}$, afforded after silica gel chromatography $(35 \%$ EtOAc in hexanes), $76.8 \mathrm{mg}(79 \%)$ of $\mathbf{1 3}$ as an off-white foam. m.p. $=67-69{ }^{\circ} \mathrm{C}$; ${ }^{1} \mathrm{H}$ NMR $\left(400 \mathrm{MHz}, \mathrm{CDCl}_{3}\right) \delta 7.84(\mathrm{~s}, 1 \mathrm{H}), 6.56(\mathrm{~d}, J=2.9 \mathrm{~Hz}, 1 \mathrm{H}), 6.33$ $(\mathrm{d}, J=2.9 \mathrm{~Hz}, 1 \mathrm{H}), 3.58(\mathrm{~s}, 2 \mathrm{H}), 3.47(\mathrm{~s}, 4 \mathrm{H}), 2.47(\mathrm{~s}, 4 \mathrm{H}), 2.16(\mathrm{~s}, 3 \mathrm{H})$, $1.46(\mathrm{~s}, 9 \mathrm{H}) ;{ }^{13} \mathrm{C} \mathrm{NMR}\left(101 \mathrm{MHz}, \mathrm{CDCl}_{3}\right) \delta 154.7,148.9,148.2,125.9,120.7,116.9,112.9,80.3$, 77.20, 61.5, 52.2, 28.4, 15.8; FTIR $\left(\mathrm{cm}^{-1}\right)$ 3347, 2976. 2938, 1477, 1365, 1248, 997; HRMS (ESI) $m / z$ $323.1959\left[(\mathrm{M}+\mathrm{H})^{+}\right.$; calcd for $\left.\mathrm{C}_{17} \mathrm{H}_{27} \mathrm{~N}_{2} \mathrm{O}_{4}{ }^{+}, 323.1965\right]$.

\section{methyl (2,5-dihydroxy-3-methylbenzyl)- $L$-prolinate (14)}<smiles>CC(=O)[C@H]1CCCN1Cc1cc(O)cc(C)c1O</smiles>

14

This reaction was performed on a $2.86 \mathrm{mmol}$ scale. According to general procedure A, 2,6-dimethyl-1,4-benzoquinone (390 mg, $2.86 \mathrm{mmol}$ ), LProline methyl ester ( $370 \mathrm{mg}, 2.86 \mathrm{mmol}, 1$ equiv) and triethylamine $(0.4$ $\mathrm{mL}, 2.86 \mathrm{mmol}, 1$ equiv) in iso-propanol $(9.5 \mathrm{~mL})$ for $2 \mathrm{~h}$ at $70{ }^{\circ} \mathrm{C}$, afforded after silica gel chromatography (40\% EtOAc in hexanes), 558 $\mathrm{mg}(76 \%)$ of 14 as a light brown oil. $100 \%$ ee as determined by HPLC (Chiracel IC, 50:50 $i$-PrOH/hexanes, $1 \mathrm{~mL} / \mathrm{min}, \lambda=290 \mathrm{~nm}$ ), $\mathrm{t}_{\mathrm{r}}$ (major) $=$ $9.1 \mathrm{~min}, \mathrm{t}_{\mathrm{r}}$ (minor) $=7.2 \mathrm{~min} .[\alpha]_{\mathrm{D}}{ }^{23}=-34.4^{\circ}\left(\mathrm{c}=1.25, \mathrm{CHCl}_{3}\right) ;{ }^{1} \mathrm{H}$ NMR $\left(400 \mathrm{MHz}, \mathrm{CDCl}_{3}\right) \delta 6.55(\mathrm{~d}, J=3.0 \mathrm{~Hz}, 1 \mathrm{H}), 6.34(\mathrm{~d}, J=2.9 \mathrm{~Hz}, 1 \mathrm{H}), 4.10(\mathrm{~d}, J=13.2 \mathrm{~Hz}, 1 \mathrm{H}), 3.74$ $(\mathrm{s}, 3 \mathrm{H}), 3.32-3.25(\mathrm{~m}, 2 \mathrm{H}), 3.01(\mathrm{ddd}, J=10.0,6.9,3.6 \mathrm{~Hz}, 1 \mathrm{H}), 2.34-2.25(\mathrm{~m}, 1 \mathrm{H}), 2.24-2.13(\mathrm{~m}$, $1 \mathrm{H}), 2.17(\mathrm{~s}, 3 \mathrm{H}), 2.01-1.90(\mathrm{~m}, 1 \mathrm{H}), 1.90-1.76(\mathrm{~m}, 2 \mathrm{H}) ;{ }^{13} \mathrm{C} \mathrm{NMR}\left(101 \mathrm{MHz}, \mathrm{CDCl}_{3}\right) \delta 174.3$, $148.9,147.7,125.8,122.4,116.7,112.6,65.3,57.4,52.9,52.3,29.6,23.3,15.9$; FTIR $\left(\mathrm{cm}^{-1}\right) 3372$, 2953, 2823, 1738, 1479, 1215, 1024; HRMS (ESI) $\mathrm{m} / z 266.1394\left[(\mathrm{M}+\mathrm{H})^{+}\right.$; calcd for $\mathrm{C}_{14} \mathrm{H}_{20} \mathrm{NO}_{4}^{+}$, 266.1392]. 


\section{2-((benzyl(methoxy)amino)methyl)-6-methylbenzene-1,4-diol (15)}<smiles>CON(Cc1ccccc1)Cc1cc(O)cc(C)c1O</smiles>

15

According to general procedure A, 2,6-dimethyl-1,4-benzoquinone (40.9 $\mathrm{mg}, 0.3 \mathrm{mmol}), \mathrm{N}$-benzyl- $O$-methylhydroxylamine $(41.7 \mathrm{mg}, 0.3 \mathrm{mmol}, 1$ equiv) and triethylamine ( $42 \mu \mathrm{L}, 0.3 \mathrm{mmol}, 1$ equiv) in iso-propanol $(1 \mathrm{~mL})$ for $2 \mathrm{~h}$ at $70{ }^{\circ} \mathrm{C}$, afforded after silica gel chromatography $(25 \%$ EtOAc in hexanes), $38.4 \mathrm{mg}(47 \%)$ of 15 as a light brown oil. ${ }^{1} \mathrm{H}$ NMR (400 MHz, $\left.\mathrm{CDCl}_{3}\right) \delta 8.85(\mathrm{~s}, 1 \mathrm{H}), 7.37-7.27(\mathrm{~m}, 5 \mathrm{H}), 6.59(\mathrm{~d}, J=3.0 \mathrm{~Hz}, 1 \mathrm{H}), 6.39$ $(\mathrm{d}, J=3.0 \mathrm{~Hz}, 1 \mathrm{H}), 4.69(\mathrm{~s}, 1 \mathrm{H}), 3.96(\mathrm{~s}, 2 \mathrm{H}), 3.87(\mathrm{~s}, 2 \mathrm{H}), 3.34(\mathrm{~s}, 3 \mathrm{H}) ;{ }^{13} \mathrm{C}$ NMR $\left(101 \mathrm{MHz}, \mathrm{CDCl}_{3}\right) \delta 148.4,148.1,135.9,129.8,128.5,127.8,126.4,121.5,117.2,113.5,61.8$, 61.3, 60.6, 15.9; FTIR $\left(\mathrm{cm}^{-1}\right)$ 3391, 3030, 2932, 1479, 1221, 1049, 1024, 775; HRMS (ESI) m/z 274.1446 [(M+H) ${ }^{+}$; calcd for $\left.\mathrm{C}_{16} \mathrm{H}_{20} \mathrm{NO}_{3}{ }^{+}, 274.1438\right]$.

\section{2-((allyl(phenyl)amino)methyl)-6-methylbenzene-1,4-diol (16)}<smiles>C=CCN(Cc1cc(O)cc(C)c1O)c1ccccc1</smiles>

According to general procedure A, 2,6-dimethyl-1,4-benzoquinone (40.9 $\mathrm{mg}, 0.3 \mathrm{mmol}), N$-allylaniline $(43 \mu \mathrm{L}, 0.3 \mathrm{mmol}, 1$ equiv) and triethylamine (42 $\mu \mathrm{L}, 0.3 \mathrm{mmol}, 1$ equiv) in iso-propanol $(1 \mathrm{~mL})$ for $2 \mathrm{~h}$ at $70{ }^{\circ} \mathrm{C}$, afforded after silica gel chromatography (20\% EtOAc in hexanes), $43 \mathrm{mg}$ $(53 \%)$ of 16 as a yellow oil. ${ }^{1} \mathrm{H}$ NMR $\left(400 \mathrm{MHz}, \mathrm{CDCl}_{3}\right) \delta 7.32-7.24(\mathrm{~m}$, 2H), $7.08(\mathrm{~m}, 2 \mathrm{H}), 7.00(\mathrm{t}, J=7.3 \mathrm{~Hz}, 1 \mathrm{H}), 6.55(\mathrm{~d}, J=2.9 \mathrm{~Hz}, 1 \mathrm{H}), 6.43$ $(\mathrm{d}, J=2.9 \mathrm{~Hz}, 1 \mathrm{H}), 5.78(\mathrm{ddt}, J=16.8,10.3,6.3 \mathrm{~Hz}, 1 \mathrm{H}), 5.22-5.08(\mathrm{~m}$, $2 \mathrm{H}), 4.28(\mathrm{~s}, 2 \mathrm{H}), 3.80(\mathrm{~d}, J=6.1 \mathrm{~Hz}, 2 \mathrm{H}), 2.18(\mathrm{~s}, 3 \mathrm{H}) ;{ }^{13} \mathrm{C}$ NMR $\left(101 \mathrm{MHz}, \mathrm{CDCl}_{3}\right) \delta 148.8,148.1$, 132.0, 129.1, 126.0, 122.4, 122.2, 119.9, 119.3, 116.7, 112.9, 55.7, 54.8, 15.8; FTIR ( $\left.\mathrm{cm}^{-1}\right) 3345,3030$, 2920, 2855, 1599, 1479, 1319, 1220, 1148, 1026, 989, 910, 858; HRMS (ESI) m/z 270.1489 [(M+H) ${ }^{+}$; calcd for $\left.\mathrm{C}_{17} \mathrm{H}_{20} \mathrm{NO}_{2}{ }^{+}, 270.1489\right]$.

\section{2-((ethyl(o-tolyl)amino)methyl)-6-methylbenzene-1,4-diol (17)}<smiles>CCN(Cc1cc(O)cc(C)c1O)c1ccccc1C</smiles>

17

According to general procedure A, 2,6-dimethyl-1,4-benzoquinone (40.9 mg, $0.3 \mathrm{mmol}), N$-ethyl-2-methylaniline (43 $\mu \mathrm{L}, 0.3 \mathrm{mmol}, 1$ equiv) and triethylamine ( $42 \mu \mathrm{L}, 0.3 \mathrm{mmol}, 1$ equiv) in iso-propanol $(1 \mathrm{~mL})$ for $2 \mathrm{~h}$ at $70{ }^{\circ} \mathrm{C}$, afforded after silica gel chromatography (18\% EtOAc in hexanes), $51.4 \mathrm{mg}(63 \%)$ of 17 as a tan solid. m.p. $=41-42{ }^{\circ} \mathrm{C} ;{ }^{1} \mathrm{H}$ NMR $(400 \mathrm{MHz}$, $\left.\mathrm{CDCl}_{3}\right) \delta 7.24-7.18(\mathrm{~m}, 3 \mathrm{H}), 7.12-7.06(\mathrm{~m}, 1 \mathrm{H}), 6.56(\mathrm{~d}, J=3.0 \mathrm{~Hz}$, $1 \mathrm{H}), 6.44(\mathrm{~d}, J=3.0 \mathrm{~Hz}, 1 \mathrm{H}), 4.12(\mathrm{~s}, 2 \mathrm{H}), 2.98(\mathrm{q}, J=7.1 \mathrm{~Hz}, 2 \mathrm{H}), 2.42$ (s, $3 \mathrm{H}), 2.20(\mathrm{~s}, 3 \mathrm{H}), 0.96(\mathrm{t}, J=7.1 \mathrm{~Hz}, 3 \mathrm{H}) ;{ }^{13} \mathrm{C} \mathrm{NMR}\left(101 \mathrm{MHz}, \mathrm{CDCl}_{3}\right) \delta$ $149.4,147.9,147.8,134.4,131.5,126.7,125.7,125.4,122.2,121.6,116.8,113.1,57.9,47.7,18.7$, 15.8, 10.7; FTIR $\left(\mathrm{cm}^{-1}\right)$ 3340, 2969, 1477, 1201 1152, 775; HRMS (ESI) $\mathrm{m} / z$ 272.1657 [(M+H) ${ }^{+}$; calcd for $\left.\mathrm{C}_{17} \mathrm{H}_{22} \mathrm{NO}_{2}^{+}, 272.1651\right]$.

\section{2-(((2-bromophenyl)(methyl)amino)methyl)-6-methylbenzene-1,4-diol (18)}<smiles>Cc1cc(O)cc(CN(C)c2ccccc2Br)c1O</smiles>

Compound 18 was prepared by a modification of general procedure A: According to general procedure A, 2,6-dimethyl-1,4-benzoquinone (40.9 $\mathrm{mg}, 0.3 \mathrm{mmol}), \mathrm{N}$-methyl-2-bromoaniline hydrochloride $(66.8,0.3 \mathrm{mmol}, 1$ equiv) and triethylamine ( $84 \mu \mathrm{L}, 0.6 \mathrm{mmol}, 2$ equiv) and iso-propanol (1 $\mathrm{mL}$ ) were combined in a vial and heated at $70^{\circ} \mathrm{C}$ for $2 \mathrm{~h}$. Concentration and silica gel chromatography (20\% EtOAc in hexanes) afforded $42.8 \mathrm{mg}$ $(40 \%)$ of 18 as a light brown oil. ${ }^{1} \mathrm{H}$ NMR $\left(400 \mathrm{MHz}, \mathrm{CDCl}_{3}\right) \delta 9.41(\mathrm{~s}$, $1 \mathrm{H}), 7.61(\mathrm{dd}, J=7.9,1.5 \mathrm{~Hz}, 1 \mathrm{H}), 7.37-7.26(\mathrm{~m}, 2 \mathrm{H}), 7.05(\mathrm{ddd}, J=8.0$, 7.1, $1.8 \mathrm{~Hz}, 1 \mathrm{H}), 6.59(\mathrm{~d}, J=2.9 \mathrm{~Hz}, 1 \mathrm{H}), 6.45(\mathrm{~d}, J=3.0 \mathrm{~Hz}, 1 \mathrm{H}), 4.67(\mathrm{~s}, 1 \mathrm{H}), 4.14(\mathrm{~s}, 2 \mathrm{H}), 2.65(\mathrm{~s}$, $3 \mathrm{H}), 2.22(\mathrm{~s}, 3 \mathrm{H}) ;{ }^{13} \mathrm{C}$ NMR $\left(101 \mathrm{MHz} \mathrm{CDCl}_{3}\right) \delta 149.9,149.0,147.8,133.8,128.5,126.6,126.3$, 122.3, 121.02, 121.0, 117.1, 113.3, 59.5, 42.6, 16.0; FTIR $\left(\mathrm{cm}^{-1}\right) 3350,3026,2959,2855,1476,1223$, 810,729 ; HRMS (ESI) $m / z 322.0428\left[(\mathrm{M}+\mathrm{H})^{+}\right.$; calcd for $\left.\mathrm{C}_{15} \mathrm{H}_{17} \mathrm{BrNO}_{2}{ }^{+}, 322.0437\right]$ 


\section{2-((5H-dibenzo[b,f]azepin-5-yl)methyl)-6-methylbenzene-1,4-diol (19)}

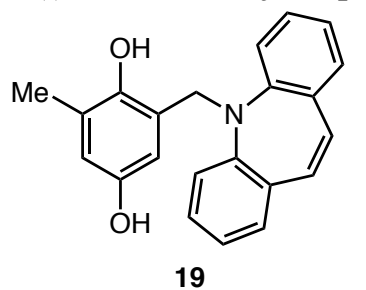

19

Compound 19 was prepared by a modification of general procedure A: According to general procedure A, 2,6-dimethyl-1,4-benzoquinone (40.9 $\mathrm{mg}, 0.3 \mathrm{mmol}), 5 H$-dibenz $[b, f]$ azepine $(58 \mathrm{mg}, 0.3 \mathrm{mmol}$, 1 equiv), triethylamine $(210 \mu \mathrm{L}, 0.3 \mathrm{mmol}, 5$ equiv $)$, iso-propanol $(0.2 \mathrm{~mL})$ and Toluene $(0.8 \mathrm{~mL})$ were combined in a vial under nitrogen and the reaction was run for $2 \mathrm{~h}$ at $70{ }^{\circ} \mathrm{C}$. Concentration and silica gel chromatography (3$4 \% \mathrm{MeCN}$ in $\mathrm{CH}_{2} \mathrm{Cl}_{2}$ ) afforded $44.8 \mathrm{mg}(45 \%)$ of 19 as an off-white solid. m.p. $=154{ }^{\circ} \mathrm{C}$ (decomp.); ${ }^{1} \mathrm{H}$ NMR $\left(400 \mathrm{MHz}, \mathrm{CDCl}_{3}\right) \delta 8.68(\mathrm{~s}, 1 \mathrm{H}), 7.27$ (m, 2H), $7.22-7.12(\mathrm{~m}, 4 \mathrm{H}), 7.05(\mathrm{t}, J=7.4 \mathrm{~Hz}, 2 \mathrm{H}), 6.95(\mathrm{~s}, 2 \mathrm{H}), 6.56(\mathrm{~d}, J=3.0 \mathrm{~Hz}, 1 \mathrm{H}), 6.42(\mathrm{~d}, J$ $=3.0 \mathrm{~Hz}, 1 \mathrm{H}), 4.90(\mathrm{~s}, 2 \mathrm{H}), 4.34(\mathrm{~s}, 1 \mathrm{H}), 2.05(\mathrm{~s}, 3 \mathrm{H}) ;{ }^{13} \mathrm{C} \mathrm{NMR}\left(101 \mathrm{MHz}, \mathrm{CDCl}_{3}\right) \delta 149.3,148.0$, 147.7, 133.2, 131.8, 129.02, 128.99, 126.3, 124.6, 121.0, 119.9, 117.0, 114.0, 77.32, 77.00, 53.6, 15.9; FTIR $\left(\mathrm{cm}^{-1}\right) 3186,1203,1150,638$; HRMS (ESI) $\mathrm{m} / z 330.1496\left[(\mathrm{M}+\mathrm{H})^{+}\right.$; calcd for $\mathrm{C}_{22} \mathrm{H}_{20} \mathrm{NO}_{2}^{+}$, 330.1494].

2-methyl-6-(((1-phenylethyl)amino)methyl)benzene-1,4-diol (20)<smiles>Cc1cc(O)cc(CNC([Na])c2ccccc2)c1O</smiles>

20 According to general procedure A, 2,6-dimethyl-1,4-benzoquinone (40.9 $\mathrm{mg}, 0.3 \mathrm{mmol})$, 1-phenylethylamine $(39 \mu \mathrm{L}, 0.3 \mathrm{mmol}, 1$ equiv) and triethylamine ( $42 \mu \mathrm{L}, 0.3 \mathrm{mmol}, 1$ equiv) in iso-propanol $(1 \mathrm{~mL})$ for $2 \mathrm{~h}$ at $70{ }^{\circ} \mathrm{C}$, afforded after silica gel chromatography $(20 \%$ and $30 \% \mathrm{MeCN}$ in $\left.\mathrm{CH}_{2} \mathrm{Cl}_{2}\right), 41.3 \mathrm{mg}(53 \%)$ of $\mathbf{2 0}$ as a light brown oil. ${ }^{1} \mathrm{H}$ NMR $(400 \mathrm{MHz}$, $\left.\mathrm{CDCl}_{3}\right) \delta 7.34(\mathrm{~m}, 2 \mathrm{H}), 7.26(\mathrm{~m}, 3 \mathrm{H}), 6.52(\mathrm{~d}, J=3.0 \mathrm{~Hz}, 1 \mathrm{H}), 6.23(\mathrm{~d}, J=$ $3.0 \mathrm{~Hz}, 1 \mathrm{H}), 5.60(\mathrm{~s}, 2 \mathrm{H}), 3.81-3.70(\mathrm{~m}, 2 \mathrm{H}), 3.59(\mathrm{~d}, J=13.7 \mathrm{~Hz}, 1 \mathrm{H})$, $2.18(\mathrm{~s}, 3 \mathrm{H}), 1.43(\mathrm{~d}, J=6.7 \mathrm{~Hz}, 3 \mathrm{H}) ;{ }^{13} \mathrm{C} \mathrm{NMR}\left(101 \mathrm{MHz}, \mathrm{CDCl}_{3}\right) \delta 149.7,147.7,143.3,128.8$, 127.5, 126.5, 126.1 122.7, 116.6, 112.6, 57.3, 50.3, 23.3, 15.8; FTIR $\left(\mathrm{cm}^{-1}\right) 3371,3318,3028,2953$, $1737,1479,1315,1217,775$; HRMS (ESI) $m / z 258.1502$ [(M+H) ${ }^{+}$; calcd for $\left.\mathrm{C}_{16} \mathrm{H}_{20} \mathrm{NO}_{2}{ }^{+}, 258.1491\right]$.

methyl (R)-2-((2,5-dihydroxy-3-methylbenzyl)amino)-2-phenylacetate (21)<smiles>COC(=O)[C@H](NCc1cc(O)cc(C)c1O)c1ccccc1</smiles>

21 Compound $\mathbf{2 1}$ was prepared by a modification of general procedure A: According to general procedure A, 2,6-dimethyl-1,4-benzoquinone (81.7 $\mathrm{mg}, 0.6 \mathrm{mmol}),(R)$-2-phenylglycine methyl ester hydrochloride (121, $0.6 \mathrm{mmol}, 1$ equiv) and triethylamine $(0.17 \mathrm{~mL}, 1.2 \mathrm{mmol}, 2$ equiv) and iso-propanol $(2 \mathrm{~mL})$ were combined in a vial and heated at $70{ }^{\circ} \mathrm{C}$ for $2 \mathrm{~h}$. The reaction was cooled down, concentrated and the residue dissolved in $\mathrm{CH}_{2} \mathrm{Cl}_{2}(15 \mathrm{~mL})$ and washed with brine $(4 \mathrm{~mL})$. The aqueous phase was extracted with $\mathrm{CH}_{2} \mathrm{Cl}_{2}(2 \times 5 \mathrm{~mL})$. Combined organic extracts were dried over $\mathrm{MgSO}_{4}$, filtered and concentrated. Silica gel chromatography $(30 \%$ EtOAc in hexanes) afforded $78 \mathrm{mg}(43 \%)$ of $\mathbf{2 1}$ as an orange oil. $39 \%$ ee as determined by HPLC (Chiracel IC, 40:60 $i-\mathrm{PrOH} /$ hexanes, $1 \mathrm{~mL} / \mathrm{min}, \lambda=290 \mathrm{~nm}$ ), $\mathrm{t}_{\mathrm{r}}$ (major) $=8.0 \mathrm{~min}, \mathrm{t}_{\mathrm{r}}$ (minor) $=6.3$ min. $[\alpha]_{\mathrm{D}}{ }^{23}=-22.0^{\circ}\left(\mathrm{c}=1.25, \mathrm{CHCl}_{3}\right) ;{ }^{1} \mathrm{H}$ NMR $\left(400 \mathrm{MHz}, \mathrm{CDCl}_{3}\right) \delta 7.39-7.27(\mathrm{~m}, 5 \mathrm{H}), 6.56(\mathrm{~d}, J=$ $3.0 \mathrm{~Hz}, 1 \mathrm{H}), 6.28(\mathrm{~d}, J=2.9 \mathrm{~Hz}, 1 \mathrm{H}), 4.40(\mathrm{~s}, 1 \mathrm{H}), 3.79$ (d, $J=4.9 \mathrm{~Hz}, 2 \mathrm{H}), 3.72(\mathrm{~s}, 3 \mathrm{H}), 2.18(\mathrm{~s}, 3 \mathrm{H})$; ${ }^{13} \mathrm{C}$ NMR $\left(101 \mathrm{MHz}, \mathrm{CDCl}_{3}\right) \delta 172.6,149.4,147.8,136.5,129.0,128.6,127.3,126.4,122.0,117.0$, 112.9, 63.7, 52.6, 50.3, 15.8; FTIR $\left(\mathrm{cm}^{-1}\right) 3317,3028,2953,1738,1479,1217,908$; HRMS (ESI) $\mathrm{m} / \mathrm{z}$ $302.1393\left[(\mathrm{M}+\mathrm{H})^{+}\right.$; calcd for $\left.\mathrm{C}_{17} \mathrm{H}_{20} \mathrm{NO}_{4}{ }^{+}, 302.1392\right]$.

\section{methyl (2,5-dihydroxy-3-methylbenzyl)- $L$-leucinate (22)}<smiles>[C+]c1cc(O)cc(NC[C@@H](CC(C)=O)C(C)=O)c1O</smiles>

22

Compound 22 was prepared by a modification of general procedure A: According to general procedure A, 2,6-dimethyl-1,4-benzoquinone (81.7 $\mathrm{mg}, 0.6 \mathrm{mmol})$, L-Leucine methyl ester hydrochloride $(109 \mathrm{mg}, 0.6$ mmol, 1 equiv) and triethylamine $(0.17 \mathrm{~mL}, 1.2 \mathrm{mmol}, 2$ equiv) and isopropanol $(2 \mathrm{~mL})$ were combined in a vial and heated at $70{ }^{\circ} \mathrm{C}$ for $2 \mathrm{~h}$. The reaction was cooled down, concentrated and the residue dissolved in $\mathrm{CH}_{2} \mathrm{Cl}_{2}(15 \mathrm{~mL})$ and washed with brine $(4 \mathrm{~mL})$. The aqueous phase was extracted with $\mathrm{CH}_{2} \mathrm{Cl}_{2}(2 \times 5 \mathrm{~mL})$. Combined organic extracts were dried

over $\mathrm{MgSO}_{4}$, filtered and concentrated. Silica gel chromatography $\left(5-10 \% \mathrm{EtOAc}\right.$ in $\left.\mathrm{CH}_{2} \mathrm{Cl}_{2}\right)$ afforded $80.6 \mathrm{mg}(48 \%)$ of 22 as a light brown oil. $100 \%$ ee as determined by HPLC (Chiracel IC, 50:50 $i$ - 
$\mathrm{PrOH} /$ hexanes, $1 \mathrm{~mL} / \mathrm{min}, \lambda=290 \mathrm{~nm}), \mathrm{t}_{\mathrm{r}}$ (major) $=6.6 \mathrm{~min}, \mathrm{t}_{\mathrm{r}}($ minor $)=17.8 \mathrm{~min} .[\alpha]_{\mathrm{D}}{ }^{24}=-22.5^{\circ}(\mathrm{c}$ $\left.=1.0, \mathrm{CHCl}_{3}\right) ;{ }^{1} \mathrm{H} \mathrm{NMR}\left(400 \mathrm{MHz}, \mathrm{CDCl}_{3}\right) \delta 6.56(\mathrm{~d}, J=2.9 \mathrm{~Hz}, 1 \mathrm{H}), 6.31(\mathrm{~d}, J=3.0 \mathrm{~Hz}, 1 \mathrm{H}), 3.92$ $(\mathrm{d}, J=13.5 \mathrm{~Hz}, 1 \mathrm{H}), 3.76(\mathrm{~s}, 3 \mathrm{H}), 3.62(\mathrm{~d}, J=13.5 \mathrm{~Hz}, 1 \mathrm{H}), 3.35(\mathrm{dd}, J=8.0,6.4 \mathrm{~Hz}, 1 \mathrm{H}), 2.18(\mathrm{~s}$, $3 \mathrm{H}), 1.75(\mathrm{td}, J=13.8,13.0,6.5 \mathrm{~Hz}, 1 \mathrm{H}), 1.50(\mathrm{ddd}, J=8.3,6.3,2.1 \mathrm{~Hz}, 2 \mathrm{H}), 0.91(\mathrm{~d}, J=6.6 \mathrm{~Hz}, 3 \mathrm{H})$, $0.87(\mathrm{~d}, J=6.5,3 \mathrm{H}) ;{ }^{13} \mathrm{C}$ NMR $\left(101 \mathrm{MHz} \mathrm{CDCl}_{3}\right) \delta 175.3,149.4,147.8,126.3,122.3,116.9,112.9$, 58.4, 52.1, 51.1, 42.4, 24.8, 22.9, 21.6, 15.8; FTIR (cm $\left.{ }^{-1}\right)$ 3316, 3020, 2957, 1732, 1479, 1217, 1149, 775; HRMS (ESI) $m / z 282.1700\left[(\mathrm{M}+\mathrm{H})^{+}\right.$; calcd for $\left.\mathrm{C}_{15} \mathrm{H}_{24} \mathrm{NO}_{4}^{+}, 282.1700\right]$.

\section{methyl $N$-benzyl- $N$-(2,5-dihydroxy-3-methylbenzyl)- $L$-leucinate (23)}<smiles>CC(=O)C(CC(C)C)N(Cc1ccccc1)Cc1cc(O)cc(C)c1O</smiles>

23

According to general procedure A, 2,6-dimethyl-1,4-benzoquinone (40.9 $\mathrm{mg}, 0.3 \mathrm{mmol}$ ), $N$-benzyl-L-Leucine methyl ester (70.6 mg, $0.3 \mathrm{mmol}, 1$ equiv) and triethylamine $(0.42 \mu \mathrm{L}, 0.3 \mathrm{mmol}, 1$ equiv) and iso-propanol ( $1 \mathrm{~mL}$ ) were combined in a vial and heated at $70{ }^{\circ} \mathrm{C}$ for $2 \mathrm{~h}$. Concentration and silica gel chromatography (25\% EtOAc in hexanes), afforded $89.5 \mathrm{mg}(71 \%)$ of $\mathbf{2 3}$ as a colorless oil. 94\% ee as determined by HPLC (Chiracel IA, 5:95 $i$-PrOH/hexanes, $1 \mathrm{~mL} / \mathrm{min}, \lambda=290 \mathrm{~nm}$ ), $\mathrm{t}_{\mathrm{r}}$ $($ major $)=14.5 \mathrm{~min}, \mathrm{t}_{\mathrm{r}}($ minor $)=17.2 \mathrm{~min} .[\alpha]_{\mathrm{D}}{ }^{22}=-70.2^{\circ}\left(\mathrm{c}=1.0, \mathrm{CHCl}_{3}\right) ;{ }^{1} \mathrm{H} \mathrm{NMR}(400 \mathrm{MHz}$, $\left.\mathrm{CDCl}_{3}\right) \delta 9.40(\mathrm{~s}, 1 \mathrm{H}), 7.37-7.27(\mathrm{~m}, 5 \mathrm{H}), 6.55(\mathrm{~d}, J=3.0 \mathrm{~Hz}, 1 \mathrm{H}), 6.39(\mathrm{~d}, J=3.0 \mathrm{~Hz}, 1 \mathrm{H}), 4.65(\mathrm{~s}$, $1 \mathrm{H}), 4.02(\mathrm{~d}, J=13.9 \mathrm{~Hz}, 1 \mathrm{H}), 3.86(\mathrm{~d}, J=13.2 \mathrm{~Hz}, 1 \mathrm{H}), 3.76(\mathrm{~s}, 3 \mathrm{H}), 3.61(\mathrm{~d}, J=13.9 \mathrm{~Hz}, 1 \mathrm{H}), 3.55$ $-3.43(\mathrm{~m}, 2 \mathrm{H}), 2.19(\mathrm{~s}, 3 \mathrm{H}), 1.81-1.69(\mathrm{~m}, 1 \mathrm{H}), 1.67-1.56(\mathrm{~m}, 2 \mathrm{H}), 0.79(\mathrm{~d}, J=6.1 \mathrm{~Hz}, 3 \mathrm{H}), 0.75$ $(\mathrm{d}, J=6.1 \mathrm{~Hz}, 3 \mathrm{H}) ;{ }^{13} \mathrm{C} \mathrm{NMR}\left(101 \mathrm{MHz}, \mathrm{CDCl}_{3}\right) \delta 172.7,148.9,147.9,137.2,129.6,128.6,127.7$, 126.0, 121.8, 116.9, 113.7, 58.9, 54.9, 53.8, 51.6, 37.6, 25.2, 22.34, 22.26, 15.9; FTIR (cm $\left.{ }^{-1}\right) 3379$, 3030, 2957, 2868, 1735, 1479, 1217, 1146, 910; HRMS (ESI) $\mathrm{m} / z$ 372.2181 [(M+H) ${ }^{+}$; calcd for $\left.\mathrm{C}_{22} \mathrm{H}_{30} \mathrm{NO}_{4}^{+}, 372.2175\right]$.

\section{2-((allyl(phenethyl)amino)methyl)-5-methoxybenzene-1,4-diol (24)}

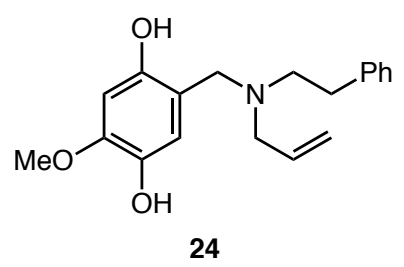

According to general procedure A, 2-methyl-5-methoxy-1,4benzoquinone (45.6 mg, $0.3 \mathrm{mmol}), \mathrm{N}$-allyl-2-phenylethylamine (48.4 $\mathrm{mg}, 0.3 \mathrm{mmol}, 1$ equiv) and triethylamine ( $42 \mu \mathrm{L}, 0.3 \mathrm{mmol}, 1$ equiv) in iso-propanol $(1 \mathrm{~mL})$ for $2 \mathrm{~h}$ at $70^{\circ} \mathrm{C}$, afforded after silica gel chromatography (25\% EtOAc in hexanes), $86.6 \mathrm{mg}(92 \%)$ of $\mathbf{2 4}$ as a light brown solid. m.p. $=62-64{ }^{\circ} \mathrm{C}$ (decomp.); ${ }^{1} \mathrm{H}$ NMR (400 MHz, $\left.\mathrm{CDCl}_{3}\right) \delta 7.35-7.27(\mathrm{~m}, 2 \mathrm{H}), 7.27-7.21(\mathrm{~m}, 1 \mathrm{H}), 7.21-7.16(\mathrm{~m}$, $2 \mathrm{H}), 6.58(\mathrm{~s}, 1 \mathrm{H}), 6.45(\mathrm{~s}, 1 \mathrm{H}), 5.90(\mathrm{ddt}, J=17.0,10.4,6.7 \mathrm{~Hz}, 1 \mathrm{H}), 5.30-5.21(\mathrm{~m}, 2 \mathrm{H}), 3.87(\mathrm{~s}, 3 \mathrm{H})$, $3.77(\mathrm{~s}, 2 \mathrm{H}), 3.25(\mathrm{~d}, J=6.7 \mathrm{~Hz}, 2 \mathrm{H}), 2.92-2.81(\mathrm{~m}, 4 \mathrm{H}) ;{ }^{13} \mathrm{C}$ NMR $\left(101 \mathrm{MHz}, \mathrm{CDCl}_{3}\right) \delta 151.2$, $139.4,138.0,133.2,128.6,128.5,126.2,119.3,114.1,113.2,100.1,56.9,55.9,55.8,54.5,32.8$; FTIR $\left(\mathrm{cm}^{-1}\right)$ 3401, 3024, 2933, 1510, 1444, 1195, 1151, 1118; HRMS (ESI) m/z 314.1765 [(M+H) ${ }^{+}$; calcd for $\left.\mathrm{C}_{19} \mathrm{H}_{24} \mathrm{NO}_{3}^{+}, 314.1756\right]$.

\section{5-((allyl(phenethyl)amino)methyl)-2,3-dimethoxybenzene-1,4-diol (25)}<smiles>C=CCN(CCc1ccccc1)Cc1cc(O)c(OC)c(OC)c1O</smiles>

25

According to general procedure A, 3,4-dimethoxy-1,4-benzoquinone (54.7 mg, $0.3 \mathrm{mmol}$ ), $N$-allyl-2-phenylethylamine (48.4 mg, $0.3 \mathrm{mmol}$, 1 equiv) and triethylamine ( $42 \mu \mathrm{L}, 0.3 \mathrm{mmol}, 1$ equiv) in iso-propanol $(1 \mathrm{~mL})$ for $2 \mathrm{~h}$ at $70{ }^{\circ} \mathrm{C}$, afforded after silica gel chromatography $(45 \%$ EtOAc in hexanes), $85.8 \mathrm{mg}(83 \%)$ of $\mathbf{2 5}$ as a light brown oil. ${ }^{1} \mathrm{H}$ NMR $\left(400 \mathrm{MHz}, \mathrm{CDCl}_{3}\right) \delta 7.62(\mathrm{~s}, 2 \mathrm{H}), 7.28(\mathrm{~m}, 2 \mathrm{H}), 7.20(\mathrm{~m}, 1 \mathrm{H}), 7.14(\mathrm{~m}$, $2 \mathrm{H}), 6.36(\mathrm{~s}, 1 \mathrm{H}), 5.89(\mathrm{ddt}, J=17.2,10.7,6.7 \mathrm{~Hz}, 1 \mathrm{H}), 5.28-5.18(\mathrm{~m}$,

$2 \mathrm{H}), 3.95(\mathrm{~s}, 3 \mathrm{H}), 3.93(\mathrm{~s}, 3 \mathrm{H}), 3.76(\mathrm{~s}, 2 \mathrm{H}), 3.23(\mathrm{dt}, J=6.7,1.3 \mathrm{~Hz}, 2 \mathrm{H}), 2.89-2.76(\mathrm{~m}, 4 \mathrm{H}) ;{ }^{13} \mathrm{C}$ NMR $\left(101 \mathrm{MHz}, \mathrm{CDCl}_{3}\right) \delta 144.8,141.2,140.7,139.7,139.4,133.2,128.6,128.5,126.3,119.5,117.5$, 108.9, 61.3, 60.6, 57.3, 56.0, 54.7, 32.9; FTIR $\left(\mathrm{cm}^{-1}\right)$ 3406, 2936, 2837, 1479, 1435, 1371, 1196, 1119 , 1074 999, 910; HRMS (ESI) $m / z$ 344.1863 [(M+H) ${ }^{+}$; calcd for $\left.\mathrm{C}_{17} \mathrm{H}_{27} \mathrm{~N}_{2} \mathrm{O}_{4}{ }^{+}, 344.1856\right]$. 


\section{2-((allyl(phenethyl)amino)methyl)-5-chlorobenzene-1,4-diol (26)}<smiles>C=CCN(CCc1ccccc1)Cc1cc(O)c(Cl)cc1O</smiles>

26

According to general procedure A, 2-chloro-5-methyl-1,4-benzoquinone (47 mg, $0.3 \mathrm{mmol}), N$-allyl-2-phenylethylamine $(48.4 \mathrm{mg}, 0.3 \mathrm{mmol}, 1$ equiv) and triethylamine (42 $\mu \mathrm{L}, 0.3 \mathrm{mmol}, 1$ equiv) in iso-propanol (1 $\mathrm{mL}$ ) for $2 \mathrm{~h}$ at $70{ }^{\circ} \mathrm{C}$, afforded after silica gel chromatography $(4 \% \mathrm{MeCN}$ in $\left.\mathrm{CH}_{2} \mathrm{Cl}_{2}\right), 33.7 \mathrm{mg}(35 \%)$ of $\mathbf{2 6}$ as a light brown oil. ${ }^{1} \mathrm{H} \mathrm{NMR}(400 \mathrm{MHz}$, $\left.\mathrm{CDCl}_{3}\right) \delta 7.28(\mathrm{~m}, 2 \mathrm{H}), 7.21(\mathrm{~m}, 1 \mathrm{H}), 7.14(\mathrm{~m}, 2 \mathrm{H}), 6.79(\mathrm{~s}, 1 \mathrm{H}), 6.65(\mathrm{~s}$, $1 \mathrm{H}), 5.86(\mathrm{ddt}, J=17.0,10.3,6.7 \mathrm{~Hz}, 1 \mathrm{H}), 5.28-5.19(\mathrm{~m}, 2 \mathrm{H}), 3.76(\mathrm{~s}$, 2H), $3.21(\mathrm{~m}, 2 \mathrm{H}), 2.88-2.76(\mathrm{~m}, 4 \mathrm{H}) ;{ }^{13} \mathrm{C} \mathrm{NMR}\left(101 \mathrm{MHz}, \mathrm{CDCl}_{3}\right) \delta$ $151.7,144.0,139.1,132.8,128.56,128.53,126.4,122.2,119.8,119.0,116.0,115.7,56.9,55.9,54.6$, 32.9; FTIR $\left(\mathrm{cm}^{-1}\right)$ 3362, 3024, 2930, 2832, 1485, 1217, 1190, 750; HRMS (ESI) $\mathrm{m} / z$ 318.1261 $\left[(\mathrm{M}+\mathrm{H})^{+}\right.$; calcd for $\left.\mathrm{C}_{18} \mathrm{H}_{21} \mathrm{NO}_{2} \mathrm{Cl}^{+}, 318.1261\right]$.

\section{2-((allyl(phenethyl)amino)methyl)-3-methylbenzene-1,4-diol (27)}<smiles>C=CCN(CCc1ccccc1)Cc1c(O)ccc(O)c1C</smiles>

27

According to general procedure A, 3,4-dimethyl-1,4-benzoquinone (40.9 mg, $0.3 \mathrm{mmol}), N$-allyl-2-phenylethylamine (48.4 $\mathrm{mg}, 0.3 \mathrm{mmol}, 1$ equiv) and triethylamine ( $42 \mu \mathrm{L}, 0.3 \mathrm{mmol}, 1$ equiv) in iso-propanol $(1 \mathrm{~mL})$ for $2 \mathrm{~h}$ at 70 ${ }^{\circ} \mathrm{C}$, afforded after silica gel chromatography (15\% EtOAc in hexanes), $52 \mathrm{mg}$ $(58 \%)$ of 27 as a light brown oil. ${ }^{1} \mathrm{H}$ NMR $\left(400 \mathrm{MHz}, \mathrm{CDCl}_{3}\right) \delta 7.32-7.24$ $(\mathrm{m}, 2 \mathrm{H}), 7.21(\mathrm{~m}, 1 \mathrm{H}), 7.15(\mathrm{~m}, 2 \mathrm{H}), 6.62(\mathrm{~d}, J=8.6,1 \mathrm{H}), 6.56(\mathrm{~d}, J=8.6$, $1 \mathrm{H}), 5.90$ (ddt, $J=17.0,10.4,6.7 \mathrm{~Hz}, 1 \mathrm{H}), 5.28-5.18(\mathrm{~m}, 2 \mathrm{H}), 3.86(\mathrm{~s}, 2 \mathrm{H})$, $3.23(\mathrm{~d}, J=6.7,2 \mathrm{H}), 2.85(\mathrm{~m}, 4 \mathrm{H}), 2.16(\mathrm{~s}, 3 \mathrm{H}) ;{ }^{13} \mathrm{C} \mathrm{NMR}\left(101 \mathrm{MHz}, \mathrm{CDCl}_{3}\right)$ $\delta 151.9,146.4,139.2,133.1,128.6,128.5,126.3,122.7,120.9,119.6,115.0,113.7,56.1,54.9,53.3$, 33.0, 11.7; FTIR $\left(\mathrm{cm}^{-1}\right)$ 3345, 3026, 2926, 1474, 1360, 1254, 1057, 925, 912, 810; HRMS (ESI) $\mathrm{m} / z$ 298.1800 [(M+H) ${ }^{+}$; calcd for $\left.\mathrm{C}_{19} \mathrm{H}_{24} \mathrm{NO}_{2}{ }^{+}, 298.1802\right]$.

\section{2-((allyl(phenethyl)amino)methyl)-3,5,6-trimethylbenzene-1,4-diol (28)}<smiles>C=CCN(CCc1ccccc1)Cc1c(C)c(O)c(C)c(C)c1O</smiles>

28

According to general procedure A, duroquinone (49.3 $\mathrm{mg}, 0.3 \mathrm{mmol}), \mathrm{N}$ allyl-2-phenylethylamine (48.4 $\mathrm{mg}, 0.3 \mathrm{mmol}, 1$ equiv) and triethylamine (42 $\mu \mathrm{L}, 0.3 \mathrm{mmol}, 1$ equiv) in iso-propanol $(1 \mathrm{~mL})$ for $6 \mathrm{~h}$ at $80{ }^{\circ} \mathrm{C}$, afforded after silica gel chromatography (15-20\% EtOAc in hexanes), $78.5 \mathrm{mg}(80 \%)$ of 28 as an orange oil. ${ }^{1} \mathrm{H}$ NMR $\left(400 \mathrm{MHz}, \mathrm{CDCl}_{3}\right) \delta 7.31$ $-7.24(\mathrm{~m}, 2 \mathrm{H}), 7.20(\mathrm{~m}, 1 \mathrm{H}), 7.14(\mathrm{~m}, 2 \mathrm{H}), 5.92(\mathrm{ddt}, J=17.2,10.6,6.7$ $\mathrm{Hz}, 1 \mathrm{H}), 5.28-5.18(\mathrm{~m}, 2 \mathrm{H}), 3.83(\mathrm{~s}, 2 \mathrm{H}), 3.23(\mathrm{~d}, J=6.7 \mathrm{~Hz}, 2 \mathrm{H}), 2.91$ - $2.75(\mathrm{~m}, 4 \mathrm{H}), 2.16(\mathrm{~s}, 9 \mathrm{H}) ;{ }^{13} \mathrm{C}$ NMR $\left(101 \mathrm{MHz}, \mathrm{CDCl}_{3}\right) \delta 149.8,144.3,139.4,133.4,128.6,128.4$, $126.2,122.3,121.4,119.3,119.2,117.3,55.9,54.6,53.2,33.0,12.8,11.8,11.7$; FTIR $\left(\mathrm{cm}^{-1}\right) 3399$, 2926, 1454, 1204, 1150; HRMS (ESI) $m / z$ 326.2131 [(M+H) ${ }^{+}$; calcd for $\left.\mathrm{C}_{21} \mathrm{H}_{28} \mathrm{NO}_{2}{ }^{+}, 326.2120\right]$.

4-((allyl(phenethyl)amino)methyl)-5-chlorobenzene-1,2-diol (29)<smiles>C=CCN(CCc1ccccc1)Cc1cc(O)c(O)cc1Cl</smiles>

29

According to general procedure A, 4-chloro-5-methyl-1,2-benzoquinone (S1, $47 \mathrm{mg}, 0.3 \mathrm{mmol}$ ), $N$-allyl-2-phenylethylamine (48.4 mg, $0.3 \mathrm{mmol}$, 1 equiv) and triethylamine ( $42 \mu \mathrm{L}, 0.3 \mathrm{mmol}, 1$ equiv) in iso-propanol (1 $\mathrm{mL})$ for $2 \mathrm{~h}$ at $70{ }^{\circ} \mathrm{C}$, afforded after silica gel chromatography (3\% MeCN in $\mathrm{CH}_{2} \mathrm{Cl}_{2}$ ), $20.8 \mathrm{mg}(22 \%)$ of 29 as a light brown oil. ${ }^{1} \mathrm{H}$ NMR (400 $\left.\mathrm{MHz} \mathrm{CDCl}_{3}\right) \delta 7.29(\mathrm{~m}, 2 \mathrm{H}), 7.21(\mathrm{t}, J=7.3 \mathrm{~Hz}, 1 \mathrm{H}), 7.14(\mathrm{~m}, 2 \mathrm{H}), 6.79$ $(\mathrm{s}, 1 \mathrm{H}), 6.65(\mathrm{~s}, 1 \mathrm{H}), 5.86(\mathrm{ddt}, J=17.0,10.3,6.7 \mathrm{~Hz}, 1 \mathrm{H}), 5.29-5.18$ $(\mathrm{m}, 2 \mathrm{H}), 3.75(\mathrm{~s}, 2 \mathrm{H}), 3.21(\mathrm{dt}, J=6.8,1.2 \mathrm{~Hz}, 2 \mathrm{H}), 2.89-2.77(\mathrm{~m}, 4 \mathrm{H}) ;{ }^{13} \mathrm{C} \mathrm{NMR}\left(101 \mathrm{MHz}, \mathrm{CDCl}_{3}\right)$ $\delta 151.7,144.0,139.1,132.8,128.56,128.54,126.4,122.2,119.8,119.0,116.0,115.7,57.0,55.9,54.6$, 32.9; FTIR $\left(\mathrm{cm}^{-1}\right)$ 3360, 3024, 2930, 2832, 1483, 1217, 1190; HRMS (ESI) m/z 318.1258 [(M+H) ${ }^{+}$; calcd for $\left.\mathrm{C}_{18} \mathrm{H}_{21} \mathrm{NO}_{2} \mathrm{Cl}^{+}, 318.1261\right]$.

\section{2-((allyl(phenethyl)amino)methyl)-5-isopropylbenzene-1,4-diol (30)}<smiles>C=CCN(CCc1ccccc1)Cc1cc(O)c(C(C)C)cc1O</smiles>

30
According to general procedure A, Thymoquinone $(49.2 \mathrm{mg}, 0.3$ mmol), $N$-allyl-2-phenylethylamine (48.4 mg, $0.3 \mathrm{mmol}, 1$ equiv) and triethylamine (42 $\mu \mathrm{L}, 0.3 \mathrm{mmol}, 1$ equiv) in iso-propanol (1 mL) for 2 $\mathrm{h}$ at $70{ }^{\circ} \mathrm{C}$, afforded after silica gel chromatography $(20 \%$ EtOAc in 
hexanes), $91.2 \mathrm{mg}(93 \%)$ of $\mathbf{3 0}$ as a light brown oil. ${ }^{1} \mathrm{H}$ NMR $\left(400 \mathrm{MHz}, \mathrm{CDCl}_{3}\right) \delta 7.31-7.24(\mathrm{~m}$, $2 \mathrm{H}), 7.23-7.18(\mathrm{~m}, 1 \mathrm{H}), 7.15(\mathrm{~d}, J=8.4 \mathrm{~Hz}, 2 \mathrm{H}), 6.67(\mathrm{~s}, 1 \mathrm{H}), 6.41$ (s, $1 \mathrm{H}), 5.87$ (ddt, $J=17.0,10.4$, $6.7 \mathrm{~Hz}, 1 \mathrm{H}), 5.26-5.16(\mathrm{~m}, 2 \mathrm{H}), 3.73(\mathrm{~s}, 2 \mathrm{H}), 3.21(\mathrm{~d}, J=6.7 \mathrm{~Hz}, 2 \mathrm{H}), 3.17-3.05(\mathrm{~m}, 1 \mathrm{H}), 2.84(\mathrm{~m}$, $4 \mathrm{H}), 1.22(\mathrm{~d}, J=6.8 \mathrm{~Hz}, 6 \mathrm{H}) ;{ }^{13} \mathrm{C}$ NMR $\left(101 \mathrm{MHz}, \mathrm{CDCl}_{3}\right) \delta 151.6,145.2,139.4,134.8,133.3,128.6$, 128.5, 126.3, 119.4, 119.3, 115.5, 113.9, 57.0, 56.0, 54.7, 32.9, 27.0, 22.61; FTIR $\left(\mathrm{cm}^{-1}\right) 3390,3026$, 2958, 1234, 1201, 1150; HRMS (ESI) $\mathrm{m} / \mathrm{z} 326.2116\left[(\mathrm{M}+\mathrm{H})^{+}\right.$; calcd for $\left.\mathrm{C}_{21} \mathrm{H}_{28} \mathrm{NO}_{2}{ }^{+}, 326.2115\right]$.

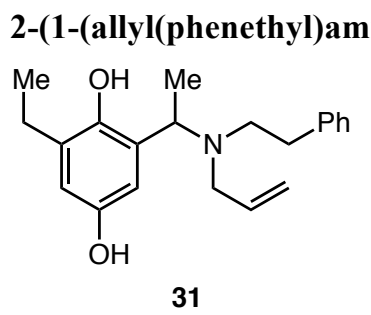

\section{Acthyl)-6-ethylbenzenc-1,4-diol (31)}

According to general procedure A, 2,6-diethyl-1,4-benzoquinone $(49.3 \mathrm{mg}$, $0.3 \mathrm{mmol}$ ), $N$-allyl-2-phenylethylamine ( $48.4 \mathrm{mg}, 0.3 \mathrm{mmol}, 1$ equiv) and triethylamine ( $42 \mu \mathrm{L}, 0.3 \mathrm{mmol}, 1$ equiv) in iso-propanol $(1 \mathrm{~mL})$ for $2 \mathrm{~h}$ at $70{ }^{\circ} \mathrm{C}$, afforded after silica gel chromatography (20\% EtOAc in hexanes), $79 \mathrm{mg}(81 \%)$ of 31 as a light yellow oil. ${ }^{1} \mathrm{H}$ NMR $\left(400 \mathrm{MHz}, \mathrm{CDCl}_{3}\right) \delta 7.31$ $-7.24(\mathrm{~m}, 2 \mathrm{H}), 7.24-7.18(\mathrm{~m}, 1 \mathrm{H}), 7.10(\mathrm{~m}, 2 \mathrm{H}), 6.60(\mathrm{~d}, J=3.0 \mathrm{~Hz}, 1 \mathrm{H})$, $6.45(\mathrm{~d}, J=3.0 \mathrm{~Hz}, 1 \mathrm{H}), 6.00-5.87(\mathrm{~m}, 1 \mathrm{H}), 5.30-5.17(\mathrm{~m}, 2 \mathrm{H}), 4.13(\mathrm{q}$, $J=6.7 \mathrm{~Hz}, 1 \mathrm{H}), 3.35$ (ddt, $J=14.1,6.1,1.5 \mathrm{~Hz}, 1 \mathrm{H}), 3.19(\mathrm{dd}, J=14.0,7.5 \mathrm{~Hz}, 1 \mathrm{H}), 2.91-2.67(\mathrm{~m}$, 4H), $2.67-2.59(\mathrm{~m}, 2 \mathrm{H}), 1.39(\mathrm{~d}, J=6.7 \mathrm{~Hz}, 3 \mathrm{H}), 1.22(\mathrm{t}, J=7.5 \mathrm{~Hz}, 3 \mathrm{H}) ;{ }^{13} \mathrm{C}$ NMR $(101 \mathrm{MHz}$, $\left.\mathrm{CDCl}_{3}\right) \delta 148.8,147.9,139.5,134.4,132.2,128.6,128.5,127.2,126.2,118.7,114.7,111.6,58.7,53.1$, 51.2, 33.7, 22.9, 14.0, 12.6; FTIR $\left(\mathrm{cm}^{-1}\right)$ 3350, 3022, 2965, 1455, 1203, 1148; HRMS (ESI) $\mathrm{m} / \mathrm{z}$ $326.2097\left[(\mathrm{M}+\mathrm{H})^{+}\right.$; calcd for $\left.\mathrm{C}_{21} \mathrm{H}_{28} \mathrm{NO}_{2}{ }^{+}, 326.2115\right]$.

tert-butyl yl)ethyl)carbamate (32)<smiles>C=CCN(CCc1ccccc1)C(CNC(=O)OCc1ccccc1)c1cc(O)c(-c2ccc(C)cc2)cc1O</smiles>

(2-(allyl(phenethyl)amino)-2-(2,5-dihydroxy-4'-methyl-[1,1'-biphenyl]-4According to general procedure A, benzoquinone S2 (102.4 mg, $0.3 \mathrm{mmol}$ ), $N$-allyl-2-phenylethylamine $(48.4 \mathrm{mg}, 0.3 \mathrm{mmol}, 1$ equiv) and triethylamine ( $42 \mu \mathrm{L}, 0.3 \mathrm{mmol}, 1$ equiv) in isopropanol $(1 \mathrm{~mL})$ for $3 \mathrm{~h}$ at $70{ }^{\circ} \mathrm{C}$, afforded after silica gel chromatography ( $5 \% \mathrm{MeCN}$ in $\left.\mathrm{CH}_{2} \mathrm{Cl}_{2}\right), 62.9 \mathrm{mg}(42 \%)$ of $\mathbf{3 2}$ as a light brown foam. $\mathrm{Mp}=90{ }^{\circ} \mathrm{C}(\mathrm{dec}) ;{ }^{1} \mathrm{H}$ NMR $\left(500 \mathrm{MHz}, \mathrm{CDCl}_{3}\right) \delta$ $7.37(\mathrm{~d}, J=8.1 \mathrm{~Hz}, 2 \mathrm{H}), 7.28(\mathrm{~m}, 4 \mathrm{H}), 7.20(\mathrm{~m}, 1 \mathrm{H}), 7.15(\mathrm{~m}, 2 \mathrm{H})$, $6.74(\mathrm{~s}, 1 \mathrm{H}), 6.60(\mathrm{~s}, 1 \mathrm{H}), 5.96-5.86(\mathrm{~m}, 1 \mathrm{H}), 5.27(\mathrm{~m}, 2 \mathrm{H}), 4.58$ $(\mathrm{t}, J=6.3 \mathrm{~Hz}, 1 \mathrm{H}), 3.94-3.82(\mathrm{~m}, 2 \mathrm{H}), 3.46-3.30(\mathrm{~m}, 3 \mathrm{H}), 3.06$ $-2.90(\mathrm{~m}, 2 \mathrm{H}), 2.90-2.79(\mathrm{~m}, 2 \mathrm{H}), 2.41(\mathrm{~s}, 3 \mathrm{H}), 1.44(\mathrm{~s}, 9 \mathrm{H}) ;{ }^{13} \mathrm{C}$ NMR $\left(126 \mathrm{MHz}, \mathrm{CDCl}_{3}\right) \delta 155.6,150.7,145.2,139.3,137.7,133.7,133.2,130.0,128.75,128.72$, 128.6, 128.3, 126.3, 124.1, 119.5, 117.9, 115.9, 79.7, 64.8, 53.0, 51.0, 40.8, 32.1, 28.4, 21.2. FTIR $\left(\mathrm{cm}^{-1}\right) 3358,3024,2978,2033,1693,1504,1454,1367,1251,1167,910$; HRMS (ESI) $\mathrm{m} / \mathrm{z} 503.2905$ $\left[(\mathrm{M}+\mathrm{H})^{+}\right.$; calcd for $\left.\mathrm{C}_{31} \mathrm{H}_{39} \mathrm{~N}_{2} \mathrm{O}_{4}^{+}, 503.2910\right]$.

4-((allyl(phenethyl)amino)methyl)-4'-fluoro-6-methyl-[1,1'-biphenyl]-2,5-diol (33a) and 6((allyl(phenethyl)amino)methyl)-4'-fluoro-4-methyl-[1,1'-biphenyl]-2,5-diol (33b)<smiles>C=CCN(CCc1ccccc1)Cc1cc(O)c(-c2ccc(F)cc2)c(C)c1O</smiles>
According to general procedure A, benzoquinone $\mathbf{S 3}(69.1 \mathrm{mg}, 0.3 \mathrm{mmol}), \mathrm{N}$ allyl-2-phenylethylamine $(48.4 \mathrm{mg}, 0.3 \mathrm{mmol}$, 1 equiv) and triethylamine ( $42 \mu \mathrm{L}, 0.3 \mathrm{mmol}$, 1 equiv) in iso-propanol $(1 \mathrm{~mL})$ for $2 \mathrm{~h}$ at 70 ${ }^{\circ} \mathrm{C}$, afforded after silica gel chromatography $\left(\mathrm{CH}_{2} \mathrm{Cl}_{2}\right), 61.5 \mathrm{mg}$ of 33a as a brown oil, 31.4 $\mathrm{mg}$ of $\mathbf{3 3 b}$ as a yellowish solid and $11.8 \mathrm{mg}$ of a mixture of 33a and 33b (ratio $=1.0: 0.37)$. Overall yield $=89 \%$. The structure of regioisomers 33a and 33b was assigned based on NOESY experiments (see below). Characterization of 33a: ${ }^{1} \mathrm{H}$ NMR ( $\left.500 \mathrm{MHz}, \mathrm{CDCl}_{3}\right) \delta 7.29-7.25(\mathrm{~m}, 2 \mathrm{H}), 7.22-7.16(\mathrm{~m}, 5 \mathrm{H}), 7.09(\mathrm{~m}, 2 \mathrm{H}), 6.72(\mathrm{~s}$, $1 \mathrm{H}), 5.80$ (ddt, $J=17.0,10.4,6.7 \mathrm{~Hz}, 1 \mathrm{H}), 5.21-5.14(\mathrm{~m}, 2 \mathrm{H}), 3.54(\mathrm{~s}, 2 \mathrm{H}), 3.13(\mathrm{dt}, J=6.8,1.3 \mathrm{~Hz}$, $2 \mathrm{H}), 2.78-2.67(\mathrm{~m}, 4 \mathrm{H}), 2.24(\mathrm{~s}, 3 \mathrm{H}) ;{ }^{13} \mathrm{C}$ NMR $\left(126 \mathrm{MHz}, \mathrm{CDCl}_{3}\right) \delta 162.4(\mathrm{~d}, J=247.7 \mathrm{~Hz}), 149.9$, $144.8,139.2,133.4,132.1$ (d, $J=8.1 \mathrm{~Hz}), 130.9$ (d, $J=3.6 \mathrm{~Hz}), 128.51,128.48,126.3,125.8,124.1$, $119.4,119.2,116.25,116.24(\mathrm{~d}, J=21.3 \mathrm{~Hz}), 56.0,54.4,54.1,32.7,15.90 .{ }^{19} \mathrm{~F}$ NMR $(377 \mathrm{MHz}$, 
$\left.\mathrm{CDCl}_{3}\right) \delta$ 113.5; FTIR $\left(\mathrm{cm}^{-1}\right)$ 3366, 3026, 2924, 2853, 1516, 1223, 734; HRMS (ESI) $\mathrm{m} / \mathrm{z} 392.2032$ $\left[(\mathrm{M}+\mathrm{H})^{+}\right.$; calcd for $\mathrm{C}_{25} \mathrm{H}_{27} \mathrm{NO}_{2} \mathrm{~F}^{+}$, 392.2026]. Characterization of 33b: m.p. $=141-144{ }^{\circ} \mathrm{C} ;{ }^{1} \mathrm{H}$ NMR $\left(500 \mathrm{MHz}, \mathrm{CDCl}_{3}\right) \delta 7.32-7.15(\mathrm{~m}, 9 \mathrm{H}), 6.51(\mathrm{~s}, 1 \mathrm{H}), 5.92(\mathrm{ddt}, J=18.1,9.5,6.7 \mathrm{~Hz}, 1 \mathrm{H}), 5.28-$ $5.23(\mathrm{~m}, 2 \mathrm{H}), 3.81(\mathrm{~s}, 2 \mathrm{H}), 3.27(\mathrm{dt}, J=6.7,1.3 \mathrm{~Hz}, 2 \mathrm{H}), 2.91-2.82(\mathrm{~m}, 4 \mathrm{H}), 1.93(\mathrm{~s}, 3 \mathrm{H}) ;{ }^{13} \mathrm{C}$ NMR $\left(126 \mathrm{MHz}, \mathrm{CDCl}_{3}\right) \delta 162.4(\mathrm{~d}, J=247.3 \mathrm{~Hz}), 149.5,145.1,139.4,133.3,132.1(\mathrm{~d}, J=8.1 \mathrm{~Hz}), 131.5$ (d, $J=3.5 \mathrm{~Hz}), 128.6,128.5,126.3,123.8,121.6,119.5,116.3(\mathrm{~d}, J=21.3 \mathrm{~Hz}), 112.3,57.3,56.1$, 54.6, 32.8, 13.2; ${ }^{19} \mathrm{~F}$ NMR (377 MHz, CDCl $) \delta 114.0$; FTIR $\left(\mathrm{cm}^{-1}\right) 3392,3026,2928,2827,1514$, 1470, 1221, 1039, 732; HRMS (ESI) $m / z$ 392.2035 [(M+H) ${ }^{+}$; calcd for $\left.\mathrm{C}_{25} \mathrm{H}_{27} \mathrm{NO}_{2} \mathrm{~F}^{+}, 392.2026\right]$.

\section{2-((allyl(phenethyl)amino)methyl)-3-(4-methoxyphenyl)naphthalene-1,4-dione (34)}

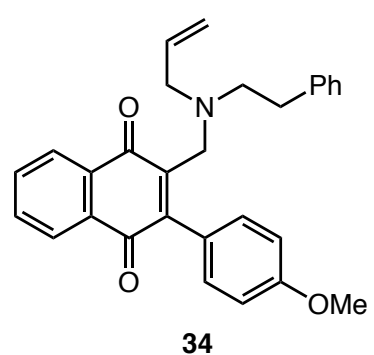

According to general procedure A, benzoquinone $\mathbf{S 4}(83.5 \mathrm{mg}, 0.3 \mathrm{mmol})$, $\mathrm{N}$-allyl-2-phenylethylamine (48.4 $\mathrm{mg}, 0.3 \mathrm{mmol}, 1$ equiv) and triethylamine ( $42 \mu \mathrm{L}, 0.3 \mathrm{mmol}, 1$ equiv) in iso-propanol $(1 \mathrm{~mL})$ for $4 \mathrm{~h}$ at $70{ }^{\circ} \mathrm{C}$, afforded after silica gel chromatography $\left(5-10 \% \mathrm{MeCN}\right.$ in $\left.\mathrm{CH}_{2} \mathrm{Cl}_{2}\right)$, $23 \mathrm{mg}(17 \%)$ of 34 as a light brown oil. ${ }^{1} \mathrm{H}$ NMR $\left(500 \mathrm{MHz}, \mathrm{CDCl}_{3}\right) \delta$ $8.14-8.09(\mathrm{~m}, 2 \mathrm{H}), 7.77-7.71(\mathrm{~m}, 2 \mathrm{H}), 7.19(\mathrm{~m}, 2 \mathrm{H}), 7.14(\mathrm{~m}, 2 \mathrm{H})$, $7.06-7.01(\mathrm{~m}, 3 \mathrm{H}), 6.96(\mathrm{~m}, 2 \mathrm{H}), 5.71(\mathrm{ddt}, J=16.8,10.1,6.5 \mathrm{~Hz}, 1 \mathrm{H})$, $5.09-5.01(\mathrm{~m}, 2 \mathrm{H}), 3.86(\mathrm{~s}, 3 \mathrm{H}), 3.60(\mathrm{~s}, 2 \mathrm{H}), 3.10(\mathrm{dt}, J=6.4,1.4 \mathrm{~Hz}$, $2 \mathrm{H}), 2.58(\mathrm{~m}, 4 \mathrm{H}) ;{ }^{13} \mathrm{C} \mathrm{NMR}\left(126 \mathrm{MHz}, \mathrm{CDCl}_{3}\right) \delta 185.6,185.0,159.9$, $147.1,143.7,140.6,135.6,133.6,133.5,132.4,132.1,131.7,128.6$, 128.1, 126.5, 126.4, 125.7, 125.0, 117.3, 113.3, 57.1, 55.29, 55.25, 49.1, 32.7; FTIR $\left(\mathrm{cm}^{-1}\right)$ 3402, 3018, 1662, 1597, 1290, 1248, 1215; HRMS (ESI) $m / z 438.2076\left[(\mathrm{M}+\mathrm{H})^{+}\right.$; calcd for $\left.\mathrm{C}_{29} \mathrm{H}_{28} \mathrm{NO}_{3}{ }^{+}, 438.2069\right]$.

\section{2-ethyl-6-(1-((1-phenylethyl)amino)ethyl)benzene-1,4-diol (35)}

$\mathrm{Me} \mathrm{OH} \mathrm{Me} \mathrm{Me} \mathrm{According} \mathrm{to} \mathrm{general} \mathrm{procedure} \mathrm{A,} \mathrm{2,6-diethyl-1,4-benzoquinone} \mathrm{(49.3} \mathrm{mg,}$ $120.3 \mathrm{mmol}), 1$-phenylethylamine $(39 \mu \mathrm{L}, 0.3 \mathrm{mmol}, 1$ equiv) and triethylamine (42 $\mu \mathrm{L}, 0.3 \mathrm{mmol}, 1$ equiv) in iso-propanol $(1 \mathrm{~mL})$ for $2 \mathrm{~h}$ at $70{ }^{\circ} \mathrm{C}$, afforded after silica gel chromatography (20-25\% EtOAc in hexanes), $40.8 \mathrm{mg}$ of the major diastereomer of $\mathbf{3 5}$ as a brown oil, $16.4 \mathrm{mg}$ of the minor diastereomer of 35 as a brown oil, and $19.5 \mathrm{mg}$ of a mixture of diastereomers (ratio 35 major:minor $=0.35: 1.0)$. Overall yield $=90 \%$. Characterization of major-35:

${ }^{1} \mathrm{H}$ NMR (400 MHz, $\left.\mathrm{CDCl}_{3}\right) \delta 7.35(\mathrm{~m}, 2 \mathrm{H}), 7.34-7.29(\mathrm{~m}, 1 \mathrm{H}), 7.15(\mathrm{~m}, 2 \mathrm{H}), 6.57(\mathrm{~d}, J=3.0 \mathrm{~Hz}$, $1 \mathrm{H}), 6.16(\mathrm{~d}, J=3.0 \mathrm{~Hz}, 1 \mathrm{H}), 3.69(\mathrm{q}, J=6.8 \mathrm{~Hz}, 1 \mathrm{H}), 3.55(\mathrm{q}, J=6.7 \mathrm{~Hz}, 1 \mathrm{H}), 2.64(\mathrm{q}, J=7.5 \mathrm{~Hz}$, $2 \mathrm{H}), 1.40(\mathrm{~d}, J=6.8 \mathrm{~Hz}, 3 \mathrm{H}), 1.32(\mathrm{~d}, J=6.8 \mathrm{~Hz}, 3 \mathrm{H}), 1.23(\mathrm{t}, J=7.5 \mathrm{~Hz}, 3 \mathrm{H}) ;{ }^{13} \mathrm{C}$ NMR $(101 \mathrm{MHz}$, $\left.\mathrm{CDCl}_{3}\right) \delta 148.8,147.9,143.7,132.5,128.8,127.5,126.6,126.3,114.5,112.3,56.2,55.4,23.5,22.75$, 22.71, 13.9; FTIR $\left(\mathrm{cm}^{-1}\right) 3310,3024,2969,1458,1375,1250,1217,768$; HRMS (ESI) $\mathrm{m} / z$ 286.1801 $\left[(\mathrm{M}+\mathrm{H})^{+}\right.$; calcd for $\left.\mathrm{C}_{18} \mathrm{H}_{24} \mathrm{NO}_{2}{ }^{+}, 286.1807\right]$. Characterization of minor-35: ${ }^{1} \mathrm{H}$ NMR $\left(400 \mathrm{MHz}, \mathrm{CDCl}_{3}\right)$ $\delta 7.35-7.29(\mathrm{~m}, 2 \mathrm{H}), 7.28-7.22(\mathrm{~m}, 3 \mathrm{H}), 6.52(\mathrm{~d}, J=3.0 \mathrm{~Hz}, 1 \mathrm{H}), 6.29(\mathrm{~d}, J=3.0 \mathrm{~Hz}, 1 \mathrm{H}), 3.92-$ $3.83(\mathrm{~m}, 2 \mathrm{H}), 2.58(\mathrm{dq}, J=11.2,7.3 \mathrm{~Hz}, 2 \mathrm{H}), 1.45(\mathrm{~d}, J=6.6 \mathrm{~Hz}, 3 \mathrm{H}), 1.40(\mathrm{~d}, J=6.6 \mathrm{~Hz}, 3 \mathrm{H}), 1.19$ $(\mathrm{t}, J=7.5 \mathrm{~Hz}, 3 \mathrm{H}) ;{ }^{13} \mathrm{C}$ NMR $\left(101 \mathrm{MHz}, \mathrm{CDCl}_{3}\right) \delta 148.6,147.7,143.4,132.6,128.6,127.4,127.3$, 126.5, 114.6, 111.8, 55.5, 54.4, 22.8, 21.9, 20.7, 13.9; FTIR $\left(\mathrm{cm}^{-1}\right)$ 3302, 3022, 2969, 1452, 1217, 767; HRMS (ESI) $m / z 286.1809\left[(\mathrm{M}+\mathrm{H})^{+}\right.$; calcd for $\left.\mathrm{C}_{18} \mathrm{H}_{24} \mathrm{NO}_{2}{ }^{+}, 286.1807\right]$.

\section{2-(10-hydroxydecyl)-5,6-dimethoxy-3-(morpholinomethyl)benzene-1,4-diol (36)}

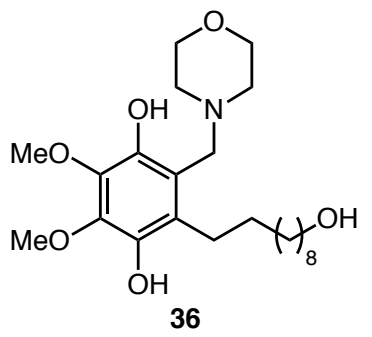

Compound $\mathbf{3 6}$ was prepared by a slight modification of general procedure A: According to general procedure A, Idebenone $(101.5 \mathrm{mg}, 0.3 \mathrm{mmol})$, morpholine $(29 \mu \mathrm{L}, 0.3 \mathrm{mmol}, 1$ equiv) and triethylamine $(42 \mu \mathrm{L}, 0.3$ mmol, 1 equiv) in iso-propanol $(1 \mathrm{~mL})$ for $6 \mathrm{~h}$ at $80^{\circ} \mathrm{C}$, afforded after silica gel chromatography $\left(25 \% \mathrm{MeCN}\right.$ in $\left.\mathrm{CH}_{2} \mathrm{Cl}_{2}\right), 94.7 \mathrm{mg}(74 \%)$ of $\mathbf{3 6}$ as a yellow oil. ${ }^{1} \mathrm{H}$ NMR $\left(400 \mathrm{MHz}, \mathrm{CDCl}_{3}\right) \delta 10.95(\mathrm{~s}, 1 \mathrm{H}), 5.47(\mathrm{~s}, 1 \mathrm{H}), 3.90$ $(\mathrm{s}, 3 \mathrm{H}), 3.88(\mathrm{~s}, 3 \mathrm{H}), 3.73(\mathrm{t}, J=4.6 \mathrm{~Hz}, 4 \mathrm{H}), 3.67(\mathrm{~s}, 2 \mathrm{H}), 3.62(\mathrm{t}, J=6.6$ $\mathrm{Hz}, 2 \mathrm{H}), 2.60-2.50(\mathrm{~m}, 6 \mathrm{H}), 1.54(\mathrm{~s}, 2 \mathrm{H}), 1.44-1.23(\mathrm{~m}, 15 \mathrm{H}) ;{ }^{13} \mathrm{C} \mathrm{NMR}$ $\left(101 \mathrm{MHz}, \mathrm{CDCl}_{3}\right) \delta 144.5,139.3,139.2,138.3,121.9,114.3,66.7,63.0$,

61.1, 60.6, 57.1, 52.7, 32.7, 30.2, 29.6, 29.5, 29.4, 29.3, 25.69, 25.66; FTIR $\left(\mathrm{cm}^{-1}\right)$ 3426, 2920, 2850, 1249, 1198, 1144, 638. 624; HRMS (ESI) $m / z 426.2854$ [(M+H) ${ }^{+}$; calcd for $\left.\mathrm{C}_{23} \mathrm{H}_{40} \mathrm{NO}_{6}{ }^{+}, 426.2850\right]$. 
2-(10-hydroxydecyl)-5,6-dimethoxy-3-(morpholinomethyl)cyclohexa-2,5-diene-1,4-dione (37)<smiles>COC1=C(OC)C(=O)C(CN2CCOCC2)=C(CC[14CH2]O)C1=O</smiles>

37

Following a reported procedure, ${ }^{4}$ to a solution of $\mathbf{3 6}(20 \mathrm{mg}, 0.047 \mathrm{mmol})$ in methanol $(1 \mathrm{~mL})$ was added a solution of $\mathrm{FeCl}_{3} \mathrm{H}_{2} \mathrm{O}(76.2 \mathrm{mg}, 0.28$ mmol, 6 equiv) in $0.1 \mathrm{M} \mathrm{HCl}(1 \mathrm{~mL})$. The mixture was stirred under at room temperature for 40 minutes and then water $(5 \mathrm{~mL})$ was added and the mixture was extracted with $\mathrm{CH}_{2} \mathrm{Cl}_{2}(4 \times 5 \mathrm{~mL})$. Combined organic extracts were washed with brine, dried over magnesium sulfate and concentrated to give $15.7 \mathrm{mg}(78 \%)$ of $\mathbf{3 7}$ as an orange oil. ${ }^{1} \mathrm{H}$ NMR $\left(400 \mathrm{MHz}, \mathrm{CDCl}_{3}\right) \delta$ $3.99(\mathrm{~s}, 3 \mathrm{H}), 3.98(\mathrm{~s}, 3 \mathrm{H}), 3.62(\mathrm{~m}, 6 \mathrm{H}), 3.34(\mathrm{~s}, 2 \mathrm{H}), 2.51(\mathrm{~m}, 2 \mathrm{H}), 2.43(\mathrm{t}$, $J=4.6 \mathrm{~Hz}, 4 \mathrm{H}), 1.55(\mathrm{~m}, 2 \mathrm{H}), 1.47-1.22(\mathrm{~m}, 15 \mathrm{H}) ;{ }^{13} \mathrm{C} \mathrm{NMR}(101 \mathrm{MHz}$, $\left.\mathrm{CDCl}_{3}\right) \delta 184.4,184.2,147.0,144.28,144.26,137.2,67.0,63.0,61.1,53.7,51.7,32.8,30.1,29.6$, 29.5, 29.37, 29.35, 29.32, 27.0, 25.7; FTIR $\left(\mathrm{cm}^{-1}\right)$ 2926, 2851, 1645, 1607, 1454, 1200, 1148, 636, 624; HRMS (ESI) $m / z 424.2684$ [(M+H) ${ }^{+}$; calcd for $\left.\mathrm{C}_{23} \mathrm{H}_{38} \mathrm{NO}_{6}{ }^{+}, 424.2694\right]$.

\section{NOESY studies for compounds $33 a$ and $33 b$}

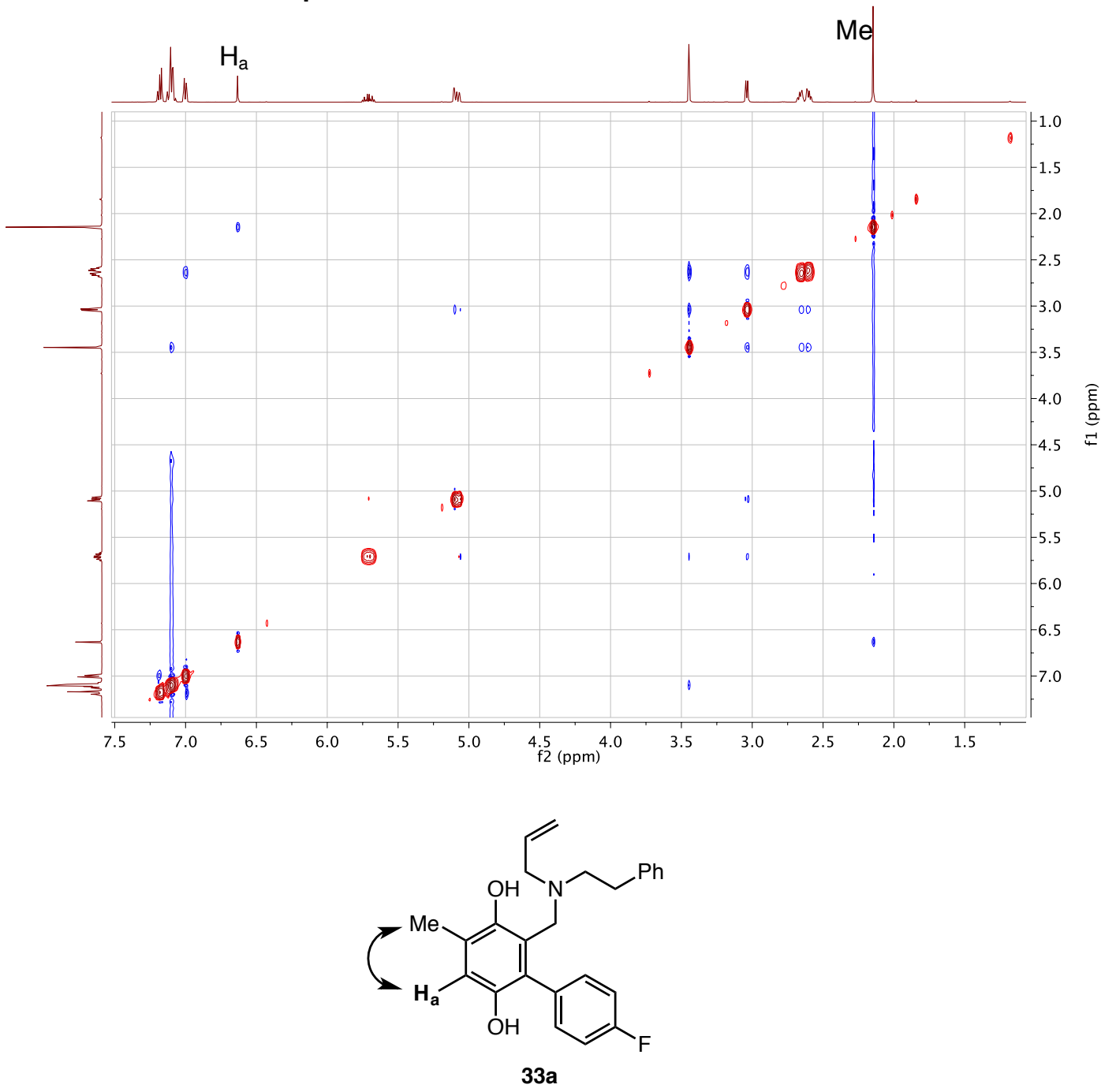

\footnotetext{
${ }^{4}$ Luly, J. R.; Rapoport, H. J. Org. Chem. 1981, 46, 2745 - 2752.
} 


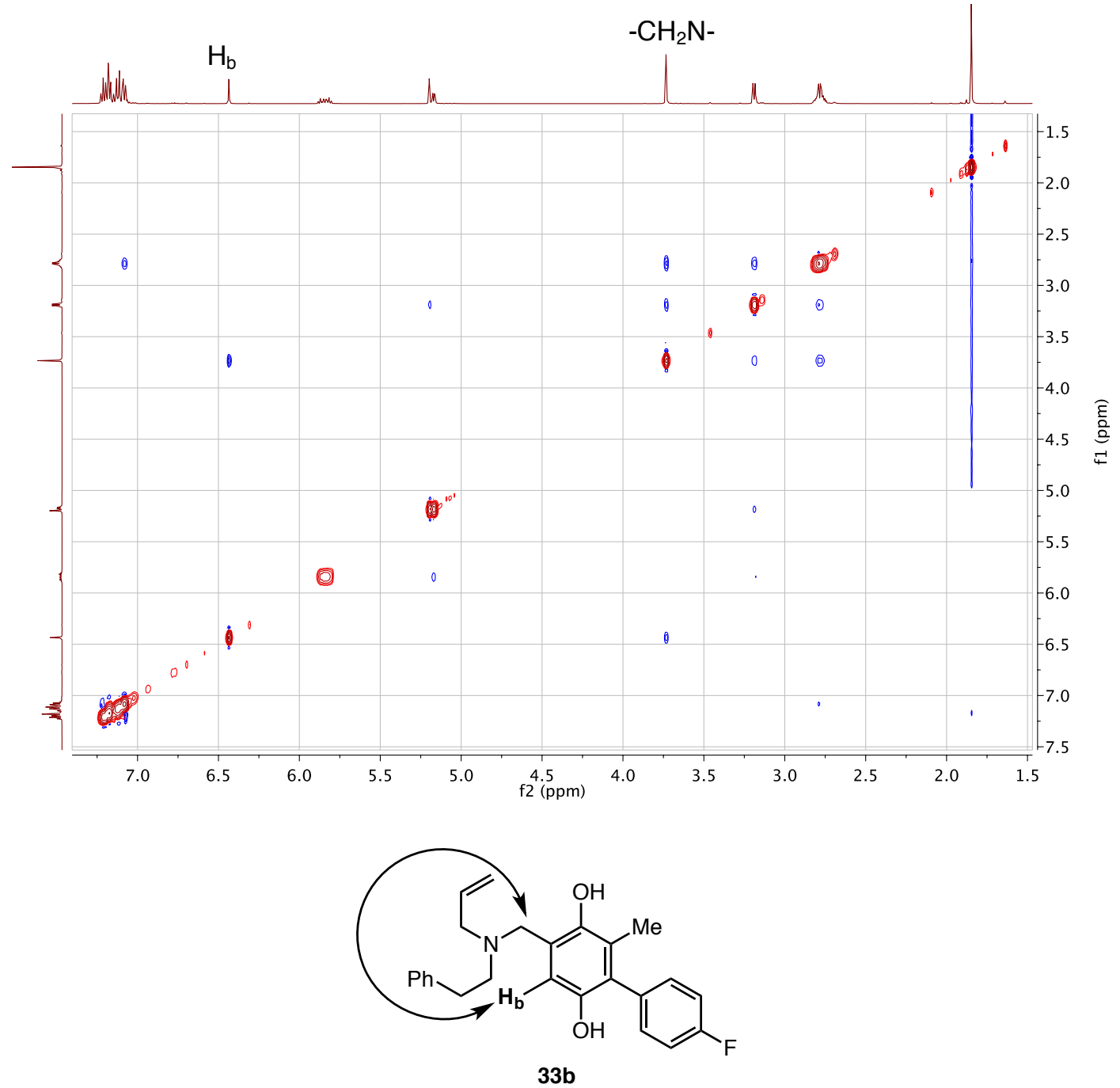




\section{HPLC traces}

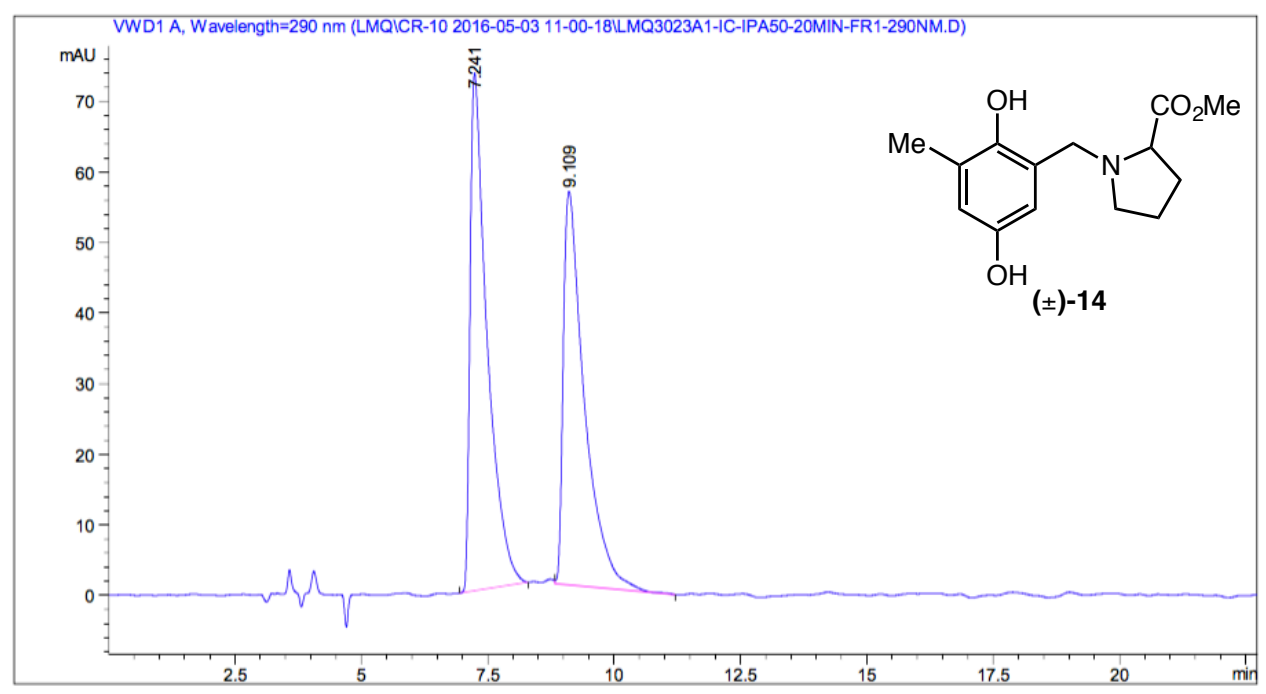

Signal 1: VWD1 A, Wavelength $=290 \mathrm{~nm}$

\begin{tabular}{|c|c|c|c|c|c|c|}
\hline $\begin{array}{c}\text { Peak } \\
\#\end{array}$ & $\begin{array}{c}\text { RetTime } \\
\text { [min] }\end{array}$ & Type & $\begin{array}{l}\text { Width } \\
\text { [min] }\end{array}$ & $\begin{array}{c}\text { Area } \\
{\left[\mathrm{mAU}^{*} \mathrm{~s}\right]}\end{array}$ & $\begin{array}{l}\text { Height } \\
\text { [mAU] }\end{array}$ & $\begin{array}{c}\text { Area } \\
\%\end{array}$ \\
\hline -1- & & & & |- - & 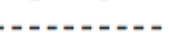 & ------ \\
\hline 1 & 7.241 & BB & 0.3341 & 1723.08789 & 73.34605 & 51.4854 \\
\hline 2 & 9.109 & VB & 0.4203 & 1623.66418 & 55.82478 & 48.5146 \\
\hline Tota] & & & & 3346.75208 & 129.17083 & \\
\hline
\end{tabular}

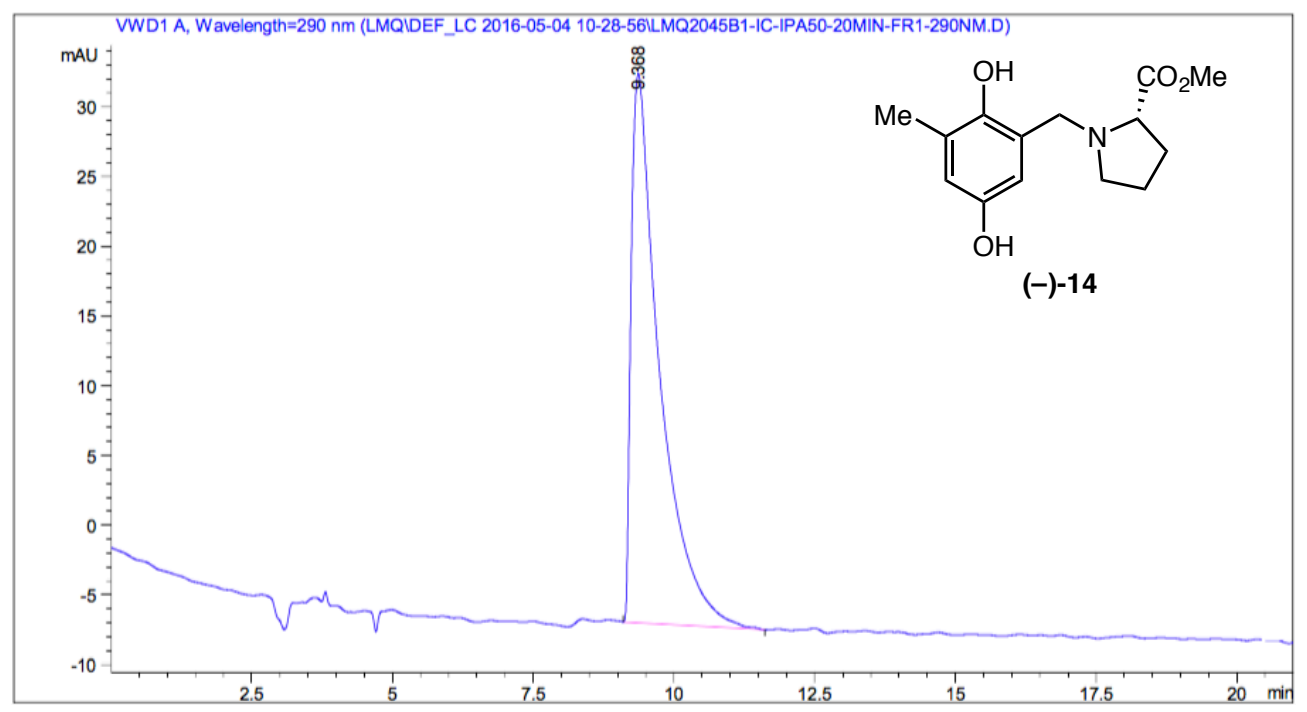

Signal 1: VWD1 A, Wavelength $=290 \mathrm{~nm}$

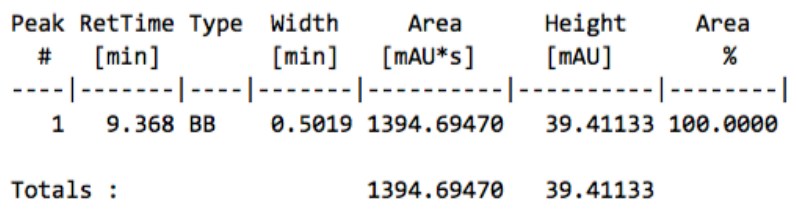




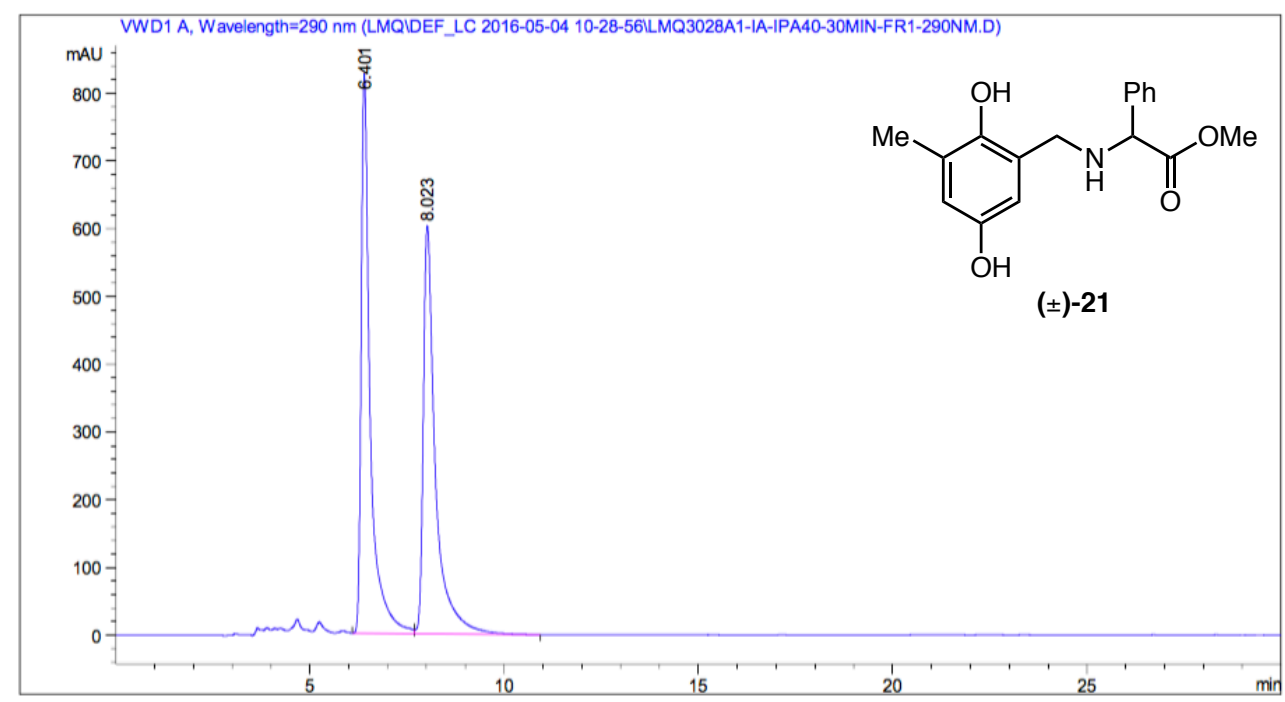

Signal 1: VWD1 A, Wavelength $=290 \mathrm{~nm}$

\begin{tabular}{llllll}
$\begin{array}{c}\text { Peak RetTime Type } \\
\text { [min] }\end{array}$ & $\begin{array}{c}\text { Width } \\
\text { [min] }\end{array}$ & $\begin{array}{c}\text { Area } \\
\text { [mAU*s] }\end{array}$ & $\begin{array}{c}\text { Height } \\
\text { [mAU] }\end{array}$ & $\begin{array}{c}\text { Area } \\
\%\end{array}$ \\
\hline 1 & 6.401 BV & 0.2326 & $1.32943 \mathrm{e} 4$ & 826.68951 & 50.5729 \\
2 & 8.023 VB & 0.3127 & $1.29931 \mathrm{e} 4$ & 602.33344 & 49.4271 \\
& & & & & \\
Totals : & & $2.62873 \mathrm{e} 4$ & 1429.02295
\end{tabular}

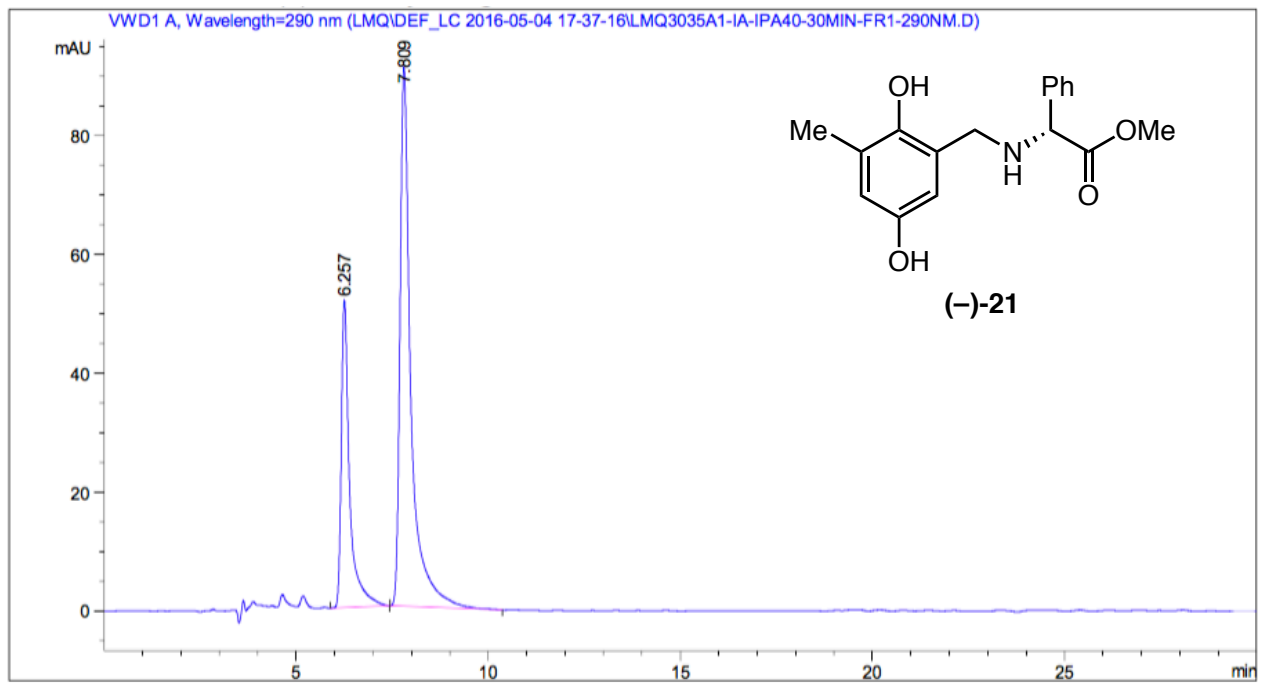

Signal 1: VWD1 A, Wavelength $=290 \mathrm{~nm}$

\begin{tabular}{|c|c|c|c|c|c|c|}
\hline $\begin{array}{c}\text { Peak } \\
\#\end{array}$ & $\begin{array}{c}\text { RetTime } \\
\text { [min] }\end{array}$ & Type & $\begin{array}{l}\text { Width } \\
\text { [min] }\end{array}$ & $\begin{array}{c}\text { Area } \\
{\left[\mathrm{mAU}^{*} \mathrm{~s}\right]}\end{array}$ & $\begin{array}{l}\text { Height } \\
\text { [mAU] }\end{array}$ & $\begin{array}{c}\text { Area } \\
\%\end{array}$ \\
\hline - & 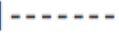 & & 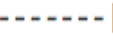 & 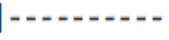 & | & - - - \\
\hline 1 & 6.257 & BB & 0.2189 & 779.42383 & 51.70963 & 30.2789 \\
\hline 2 & 7.809 & BB & 0.2866 & 1794.72449 & 90.78149 & 69.7211 \\
\hline Tota] & Is : & & & 2574.14832 & 142.49111 & \\
\hline
\end{tabular}




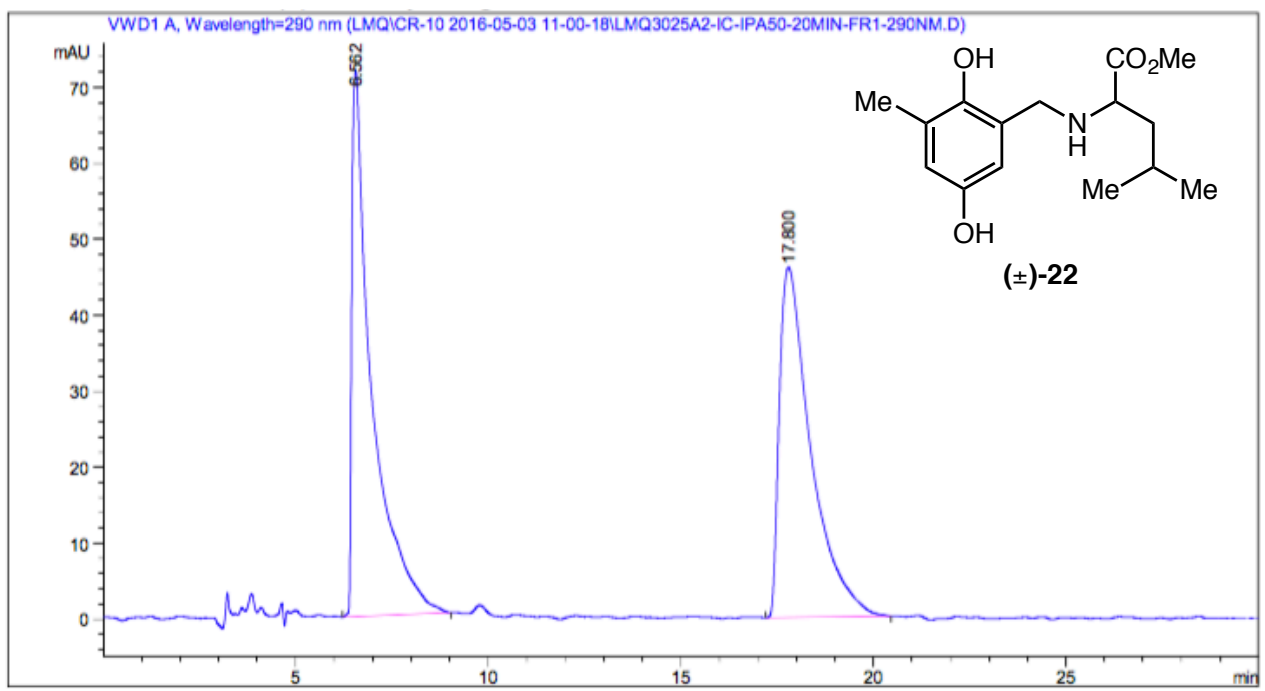

Signal 1: VWD1 A, Wavelength $=290 \mathrm{~nm}$

\begin{tabular}{|c|c|c|c|c|c|}
\hline $\begin{array}{c}\text { Peak } \\
\text { \# }\end{array}$ & $\begin{array}{l}\text { RetTime Type } \\
\text { [min] }\end{array}$ & $\begin{array}{l}\text { Width } \\
\text { [min] }\end{array}$ & $\begin{array}{c}\text { Area } \\
{[\mathrm{mAU} * \mathrm{~s}]}\end{array}$ & $\begin{array}{l}\text { Height } \\
{[\mathrm{MAU}]}\end{array}$ & $\begin{array}{c}\text { Area } \\
\%\end{array}$ \\
\hline & & & 2599.68872 & 71. 78978 & \\
\hline 2 & $17.800 \mathrm{BB}$ & 0.8441 & 2626.95947 & 46.08073 & 50.2609 \\
\hline
\end{tabular}

Totals :

$5226.64819 \quad 117.87051$

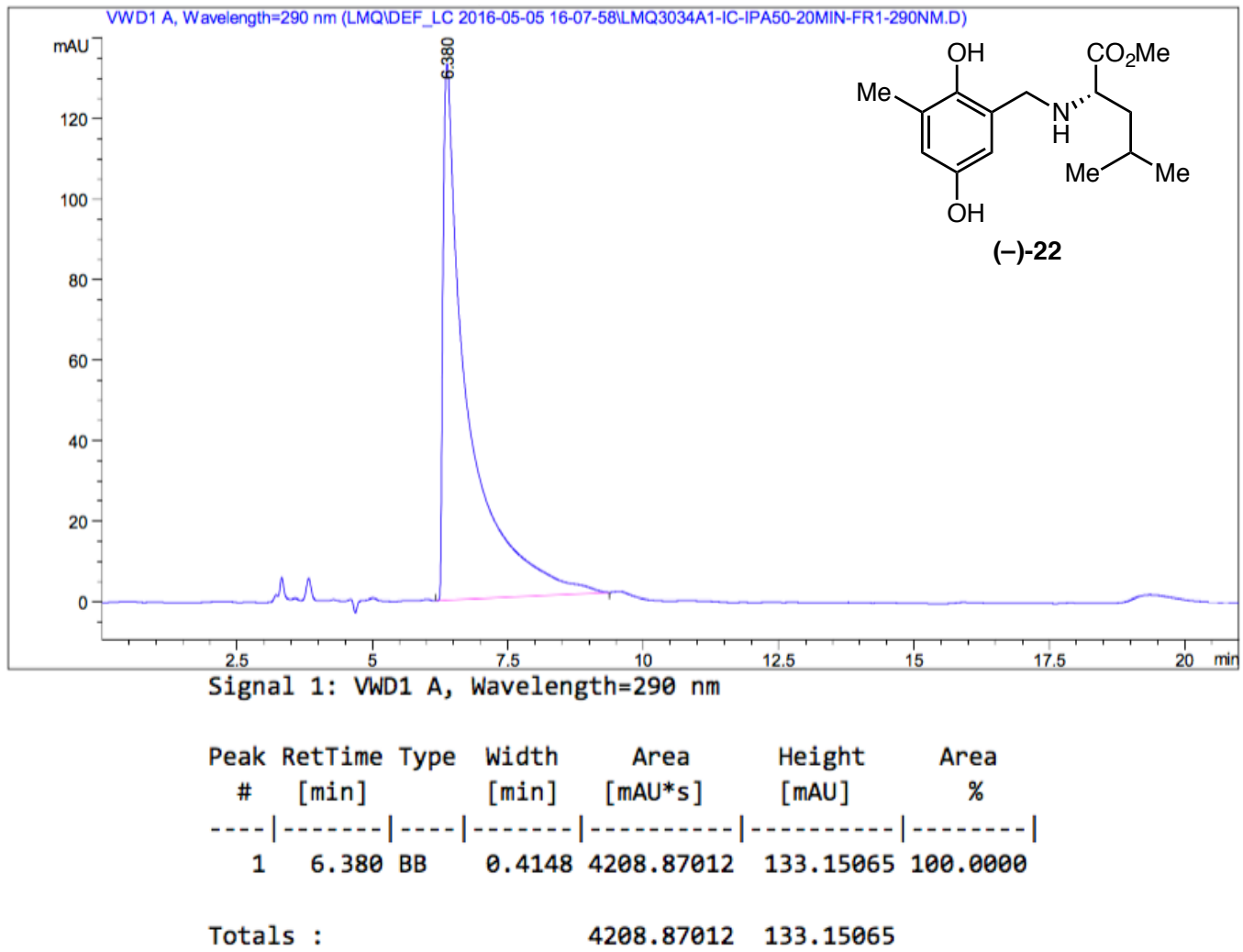


Note: we were unable to obtain a clean chromatogram of compound 23 (both racemic and enantioenriched) due to its propensity to oxidize upon isolation.
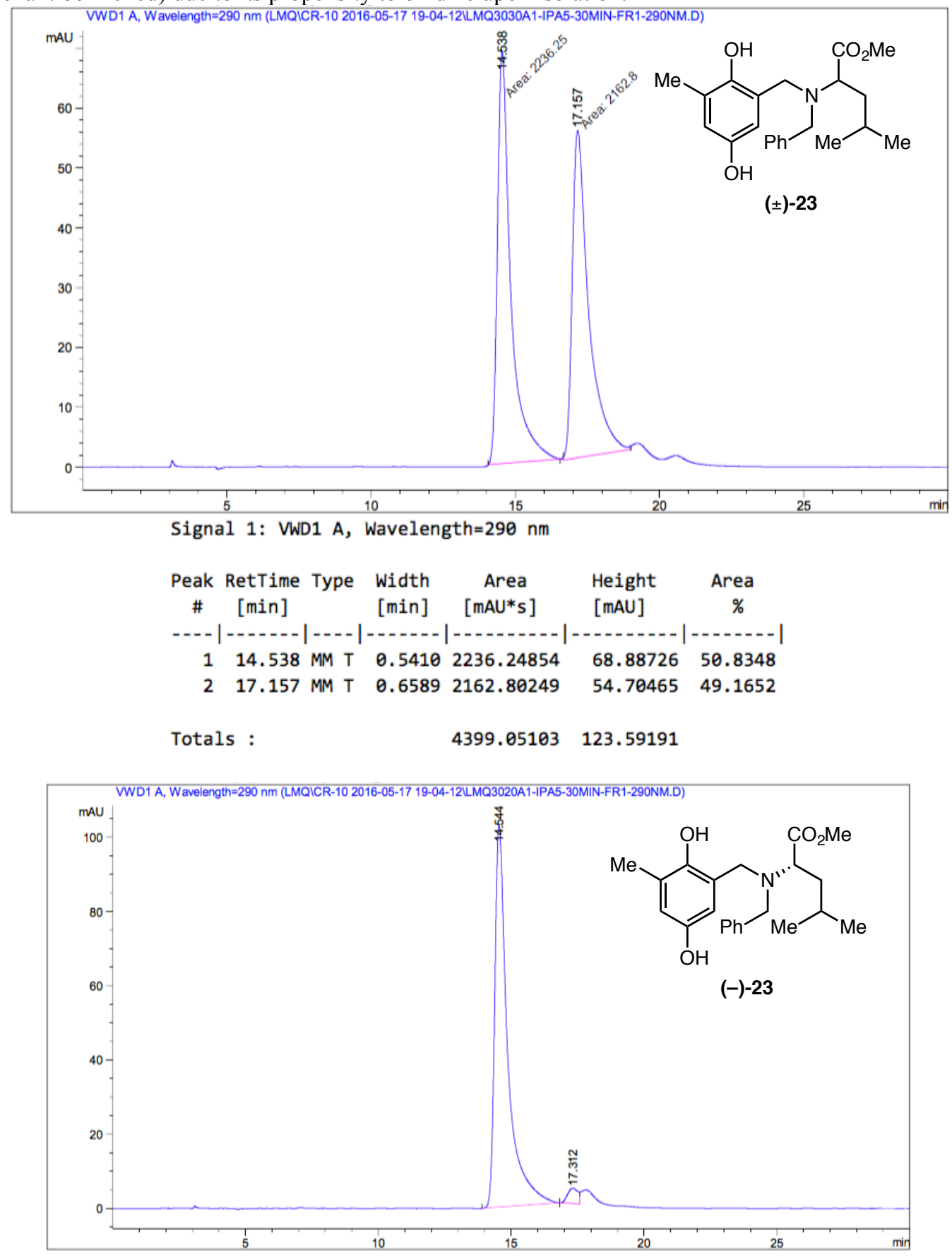

Signal 1: VWD1 A, Wavelength $=290 \mathrm{~nm}$

\begin{tabular}{|c|c|c|c|c|c|}
\hline $\begin{array}{c}\text { Peak } \\
\#\end{array}$ & $\begin{array}{l}\text { RetTime Type } \\
\text { [min] }\end{array}$ & $\begin{array}{l}\text { Width } \\
\text { [min] }\end{array}$ & $\begin{array}{c}\text { Area } \\
{\left[\mathrm{mAU}^{*} \mathrm{~s}\right]}\end{array}$ & $\begin{array}{l}\text { Height } \\
\text { [mAU] }\end{array}$ & $\begin{array}{c}\text { Area } \\
\%\end{array}$ \\
\hline$=-1$ & & & $\mid-\ldots-$ & - & | \\
\hline 1 & 14.544 ВВ & 0.4869 & 3470.98096 & 103.06225 & 96.9128 \\
\hline 2 & $17.312 \mathrm{BV}$ & 0.4128 & 110.56849 & 4.04665 & 3.0872 \\
\hline Tota] & Ls : & & 3581.54945 & 107.10890 & \\
\hline
\end{tabular}


7. Copies of ${ }^{1} \mathrm{H},{ }^{13} \mathrm{C}$ and ${ }^{19} \mathrm{~F}$ NMR Spectra
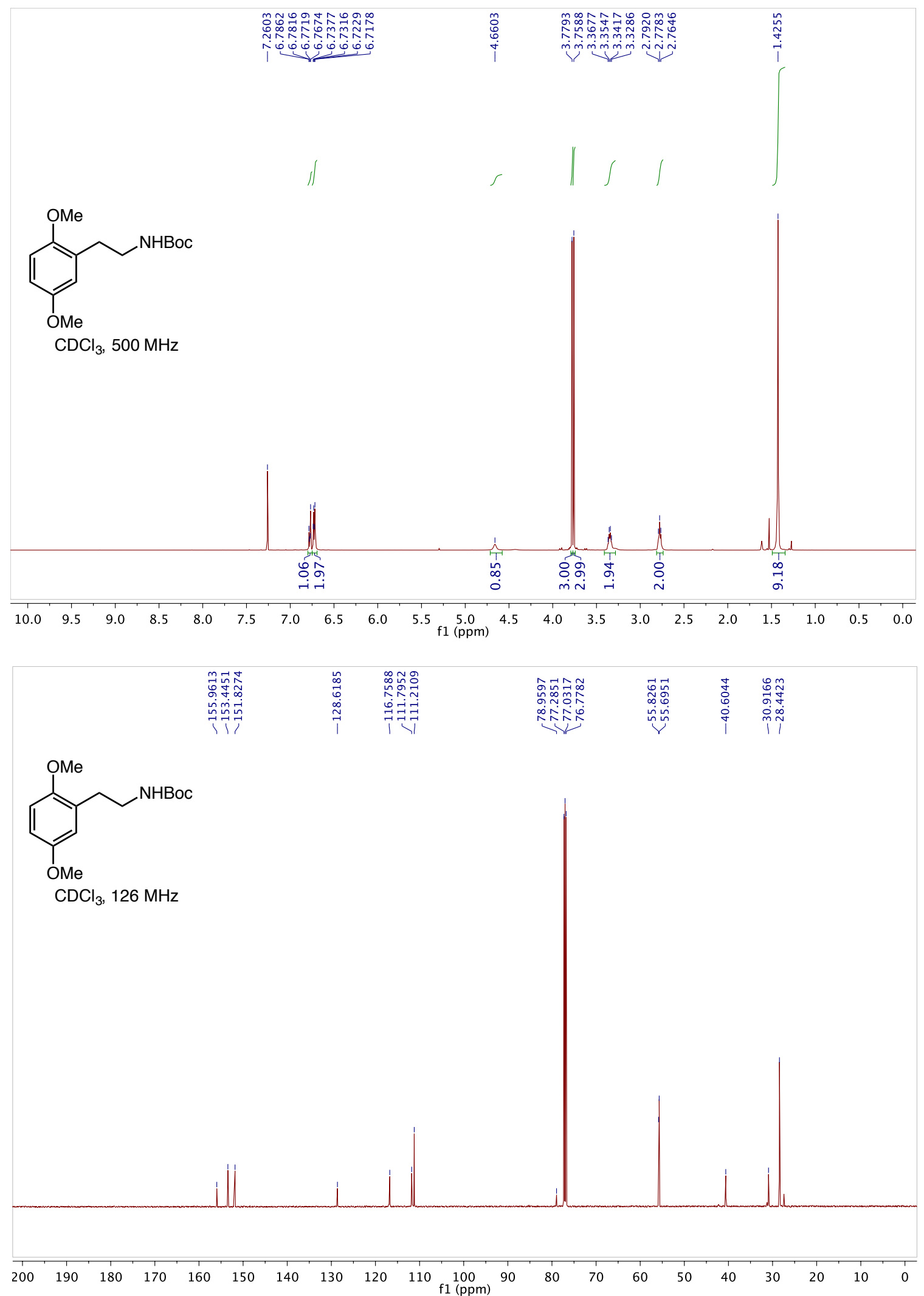


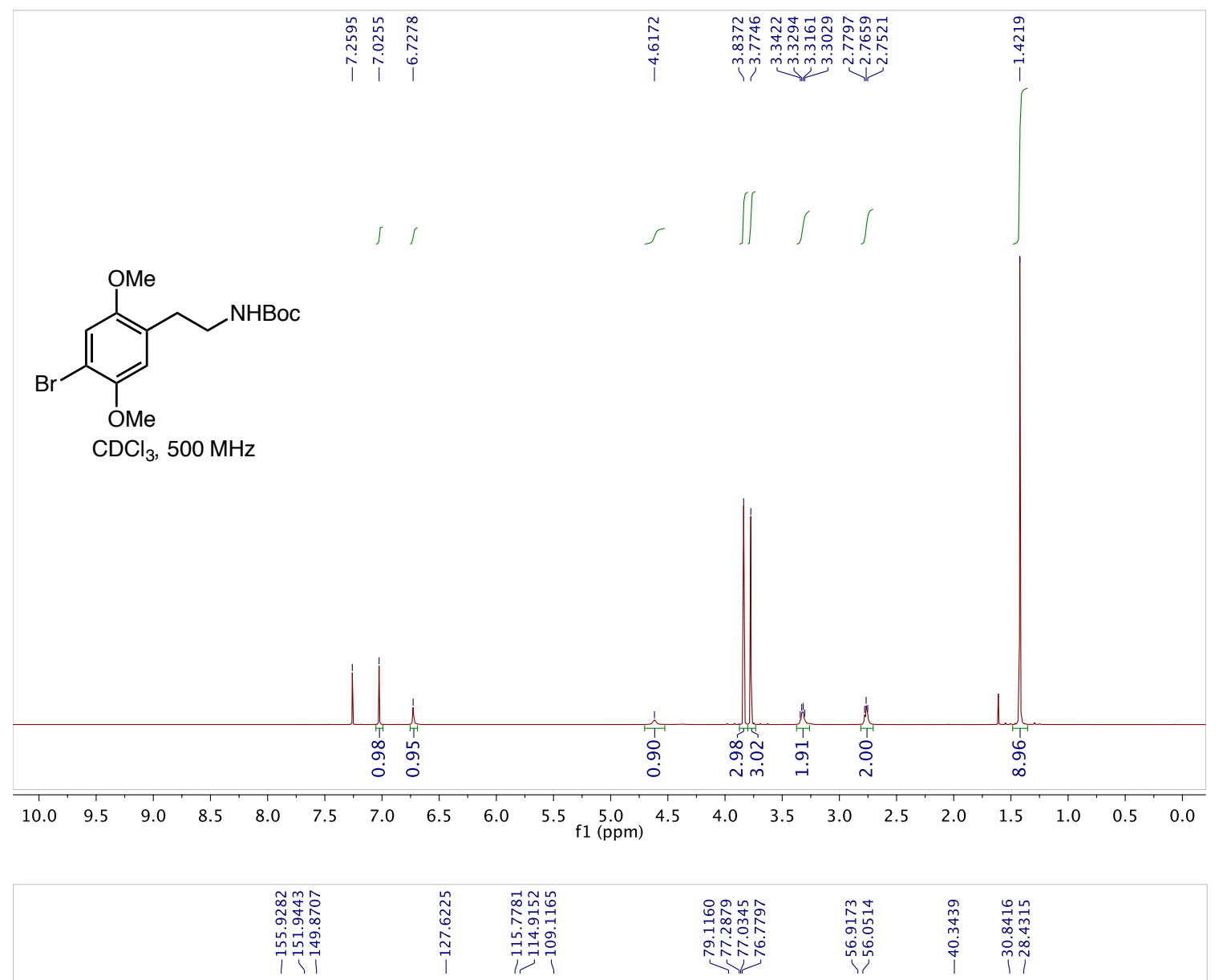<smiles>COc1cc(CCNC(=O)OCc2ccccc2)c(OC)cc1Br</smiles>
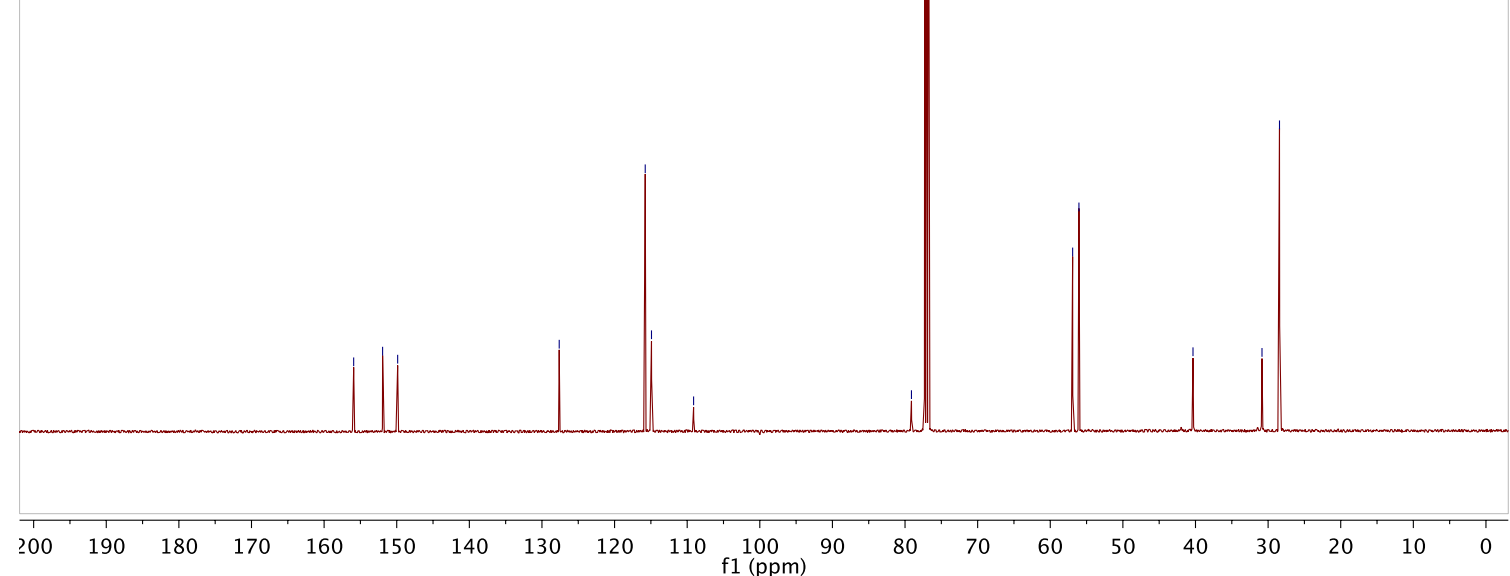


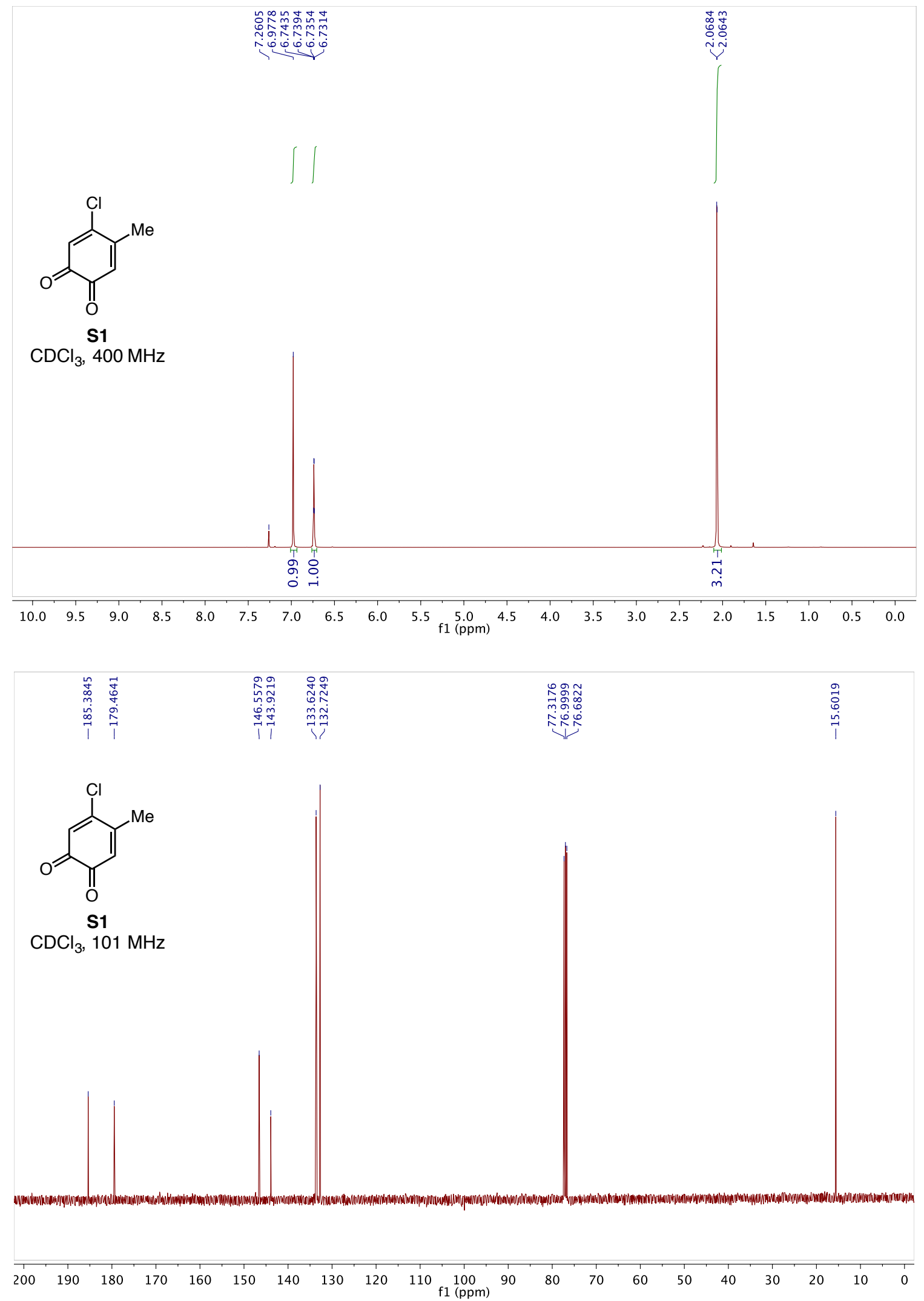



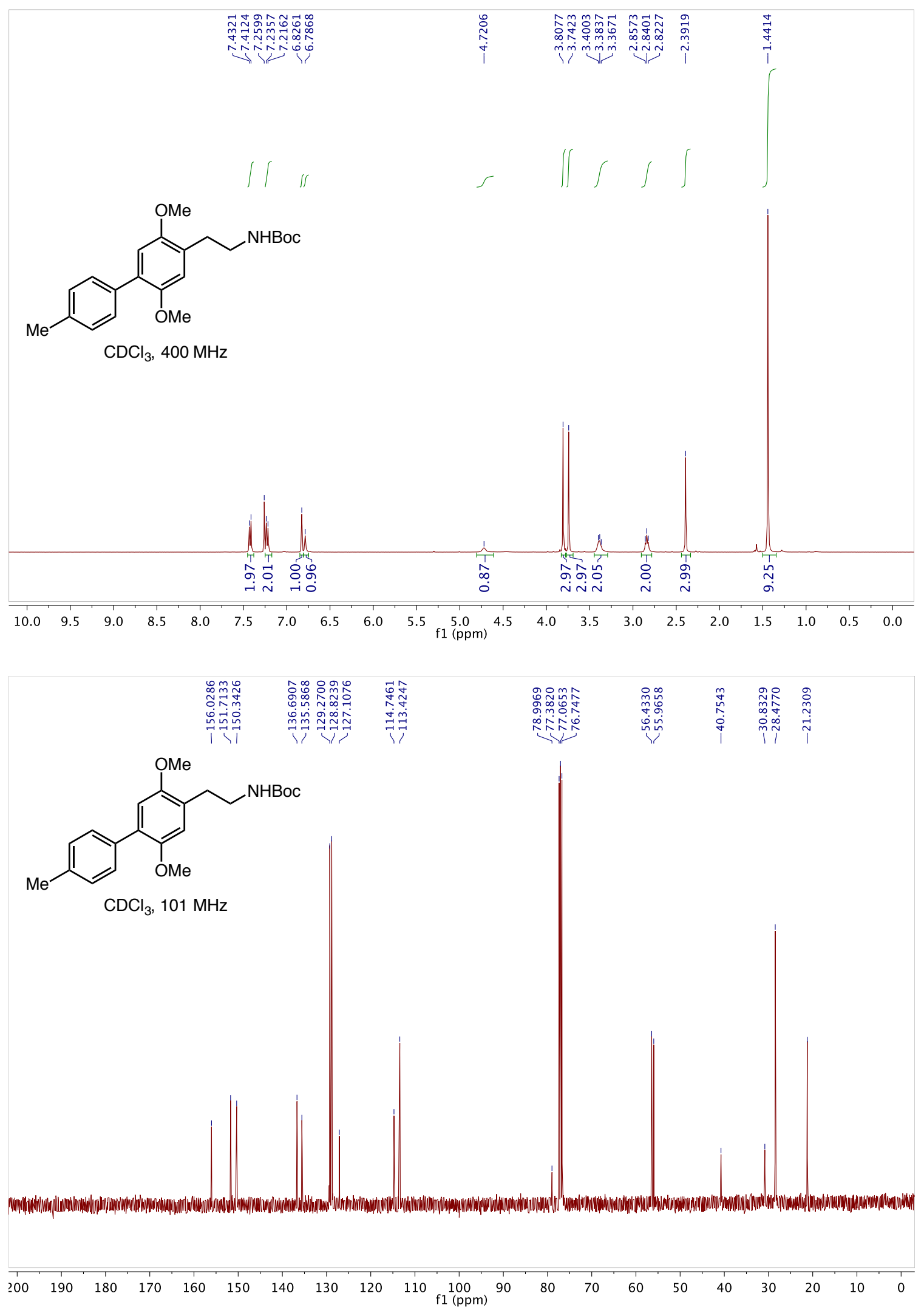

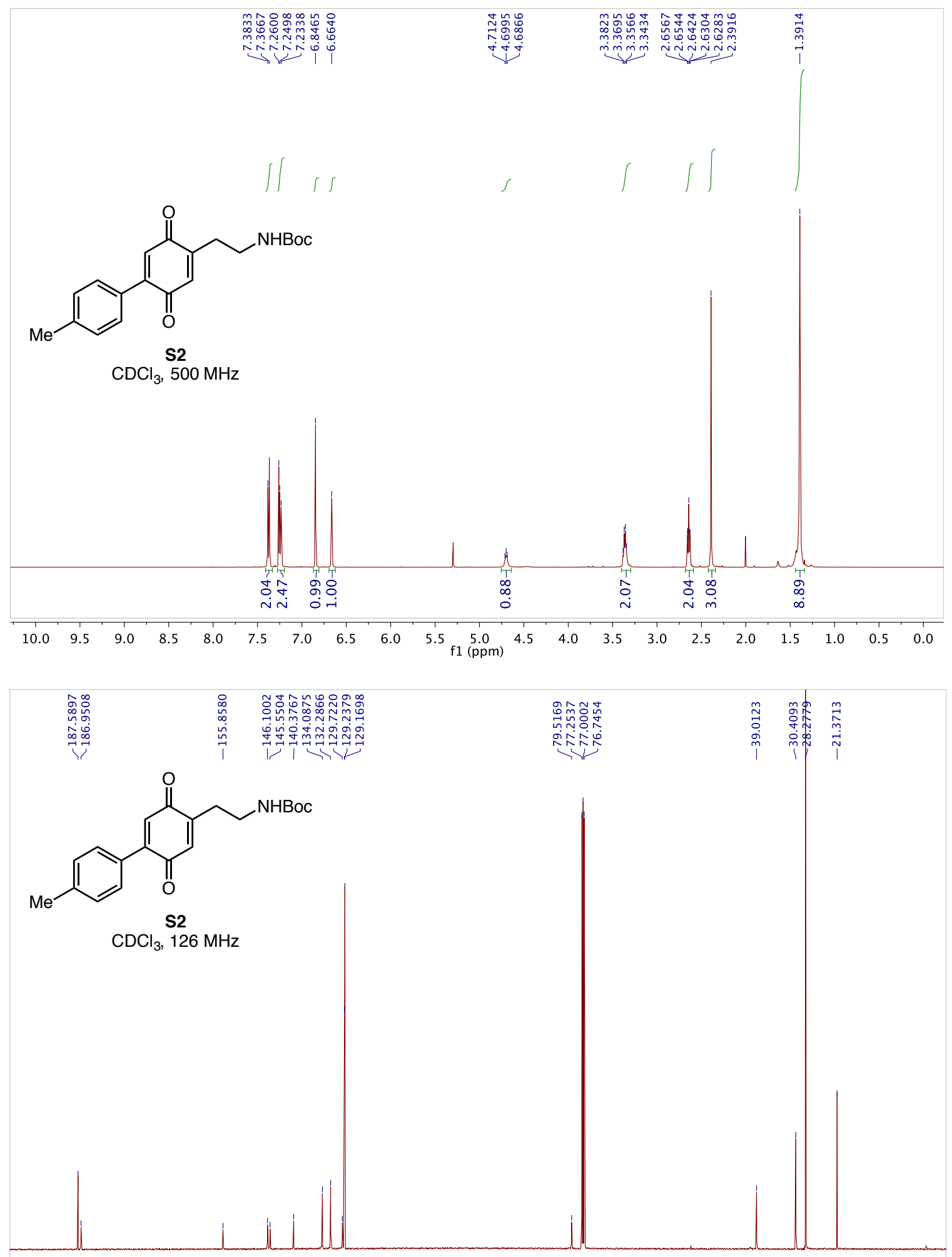

$\begin{array}{lllllllllllllllllllll}200 & 190 & 180 & 170 & 160 & 150 & 140 & 130 & 120 & 110 & 100 & 90 & 80 & 70 & 60 & 50 & 40 & 30 & 20 & 10 & 0\end{array}$ 

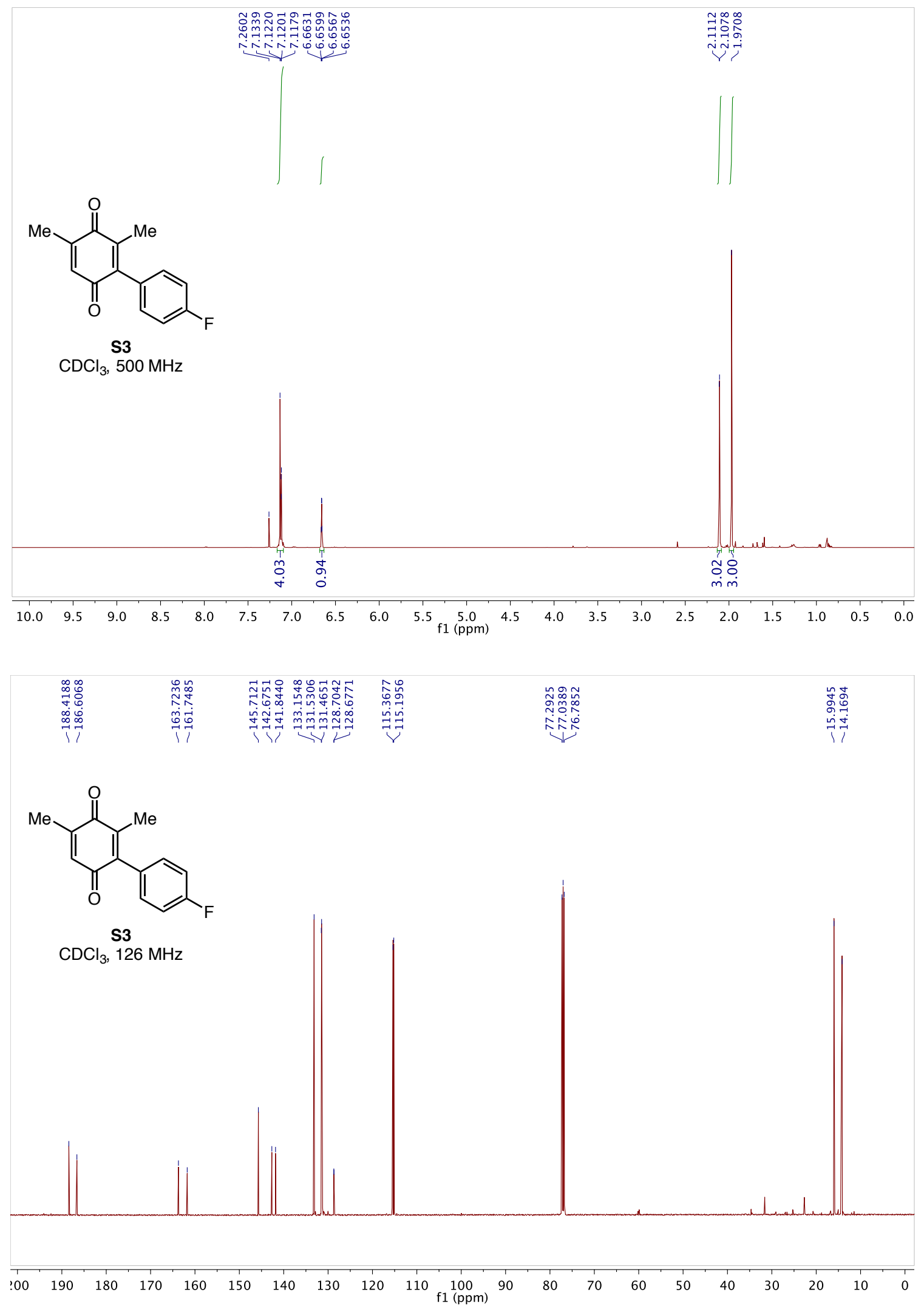


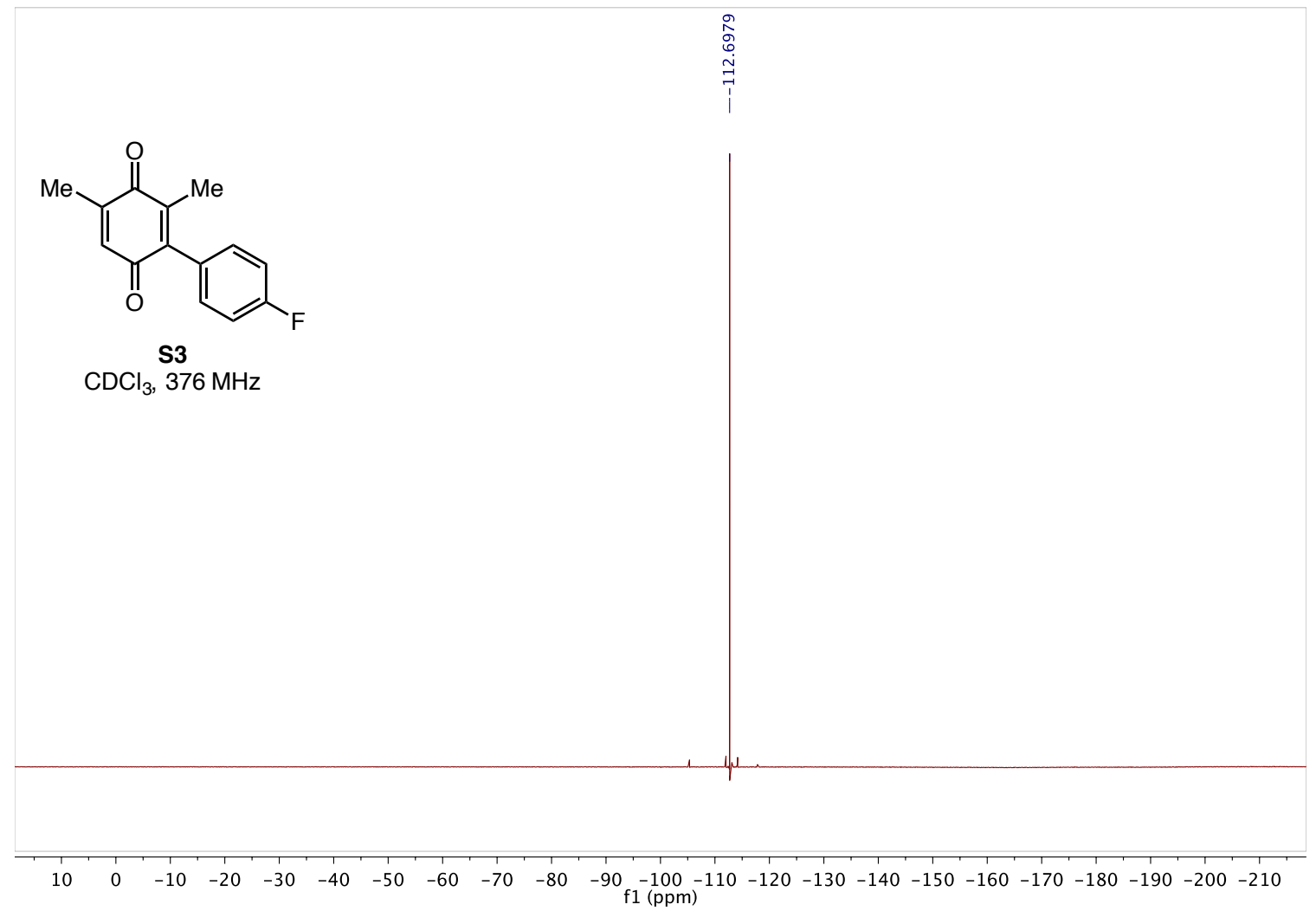




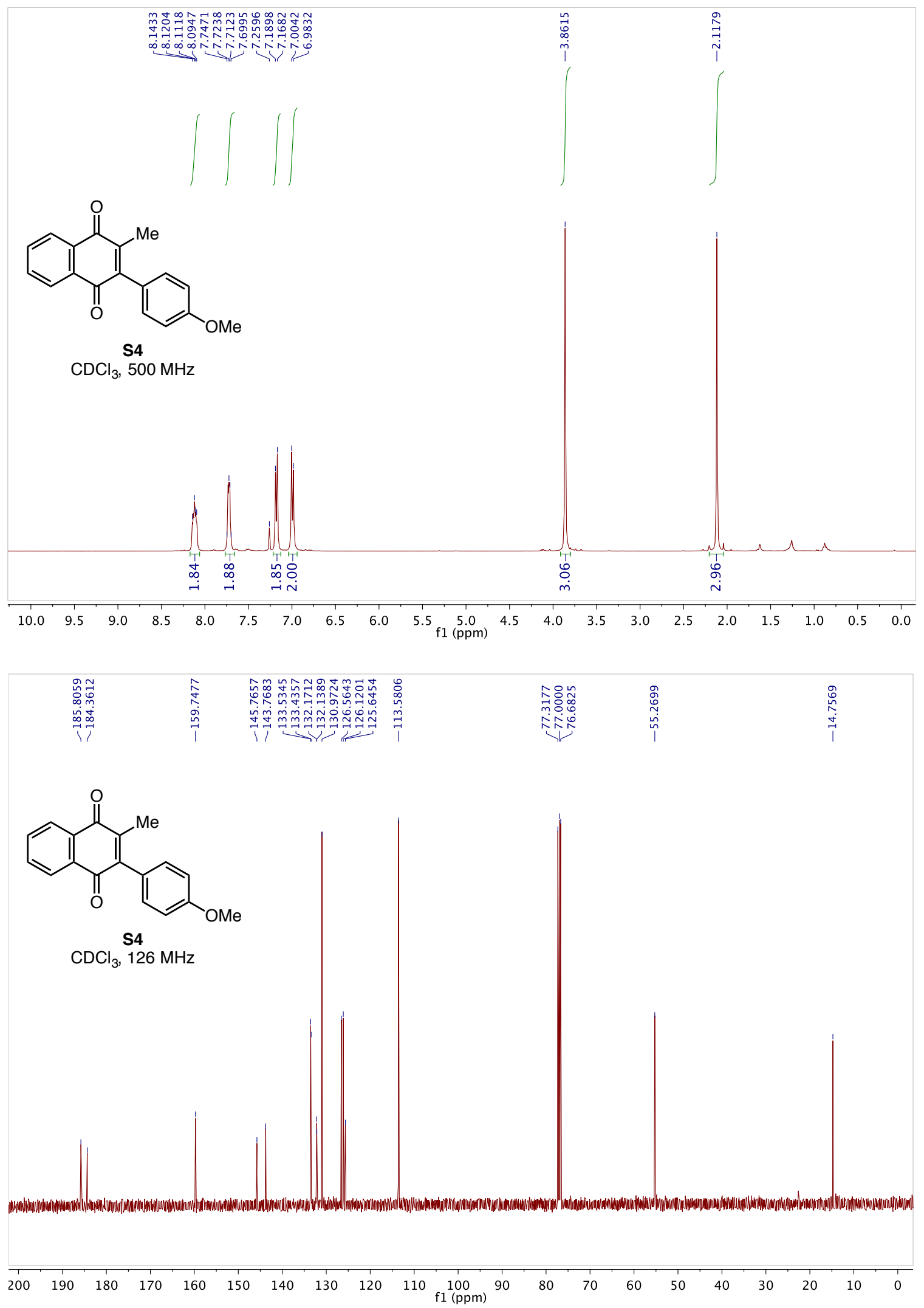




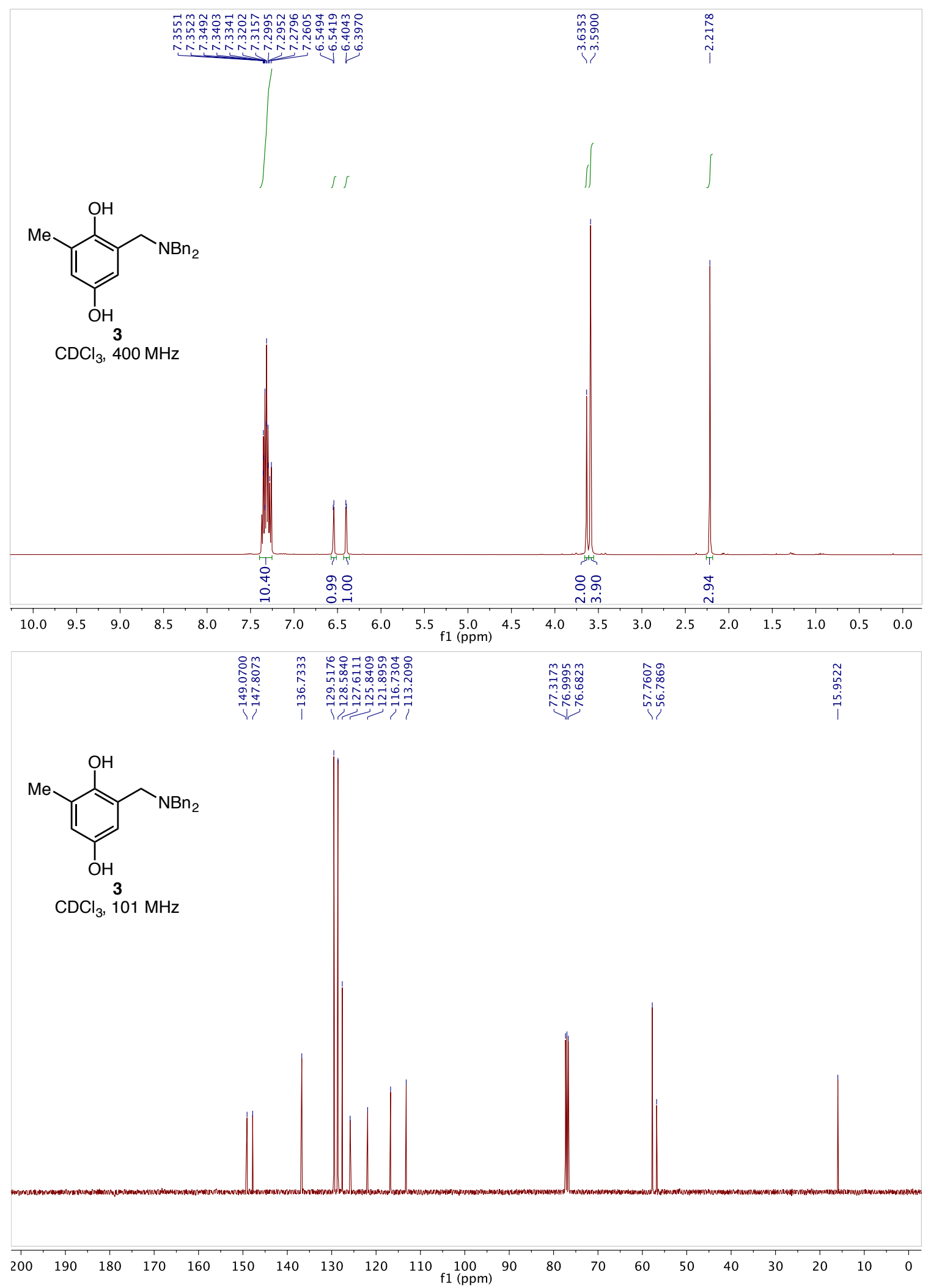




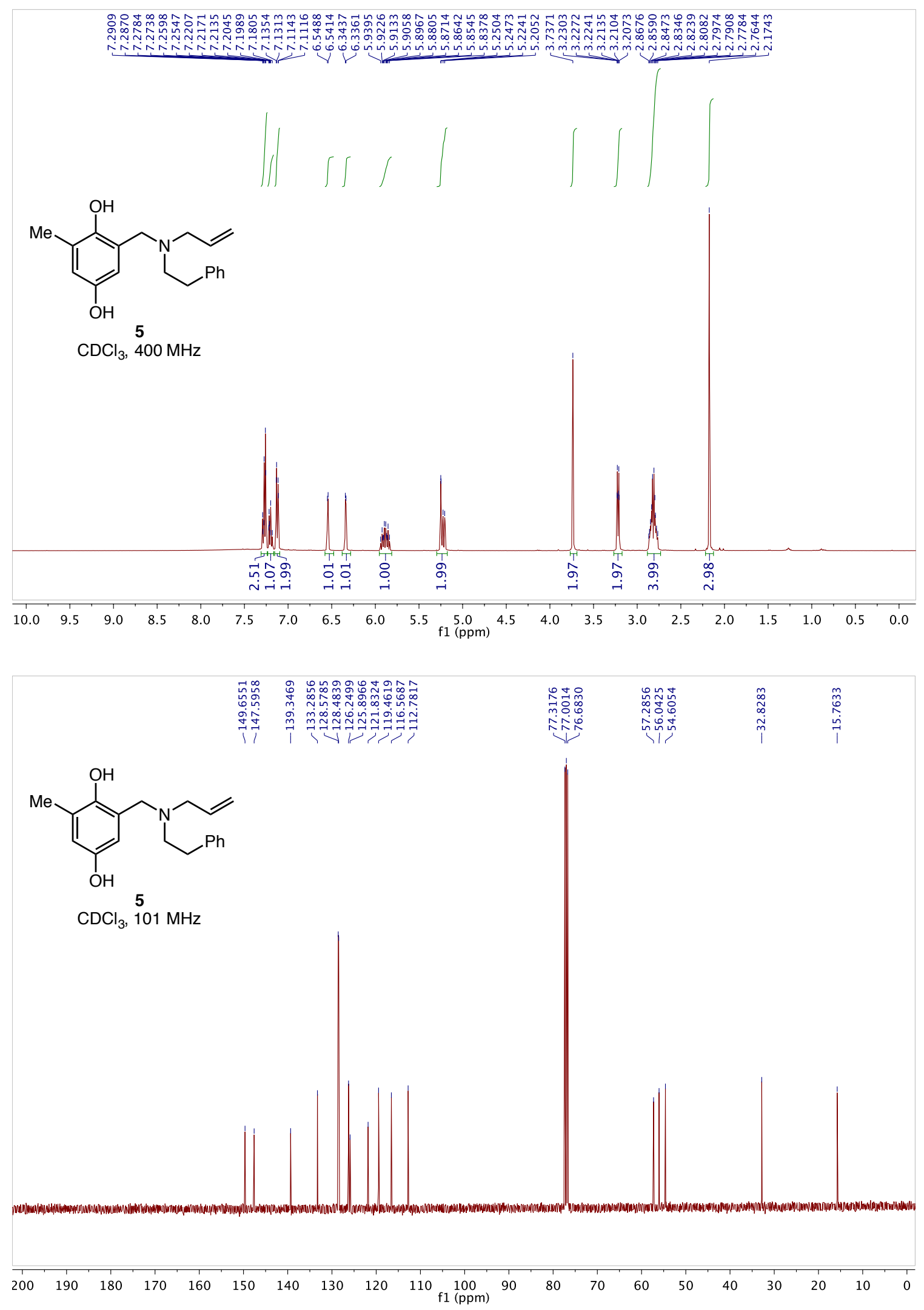




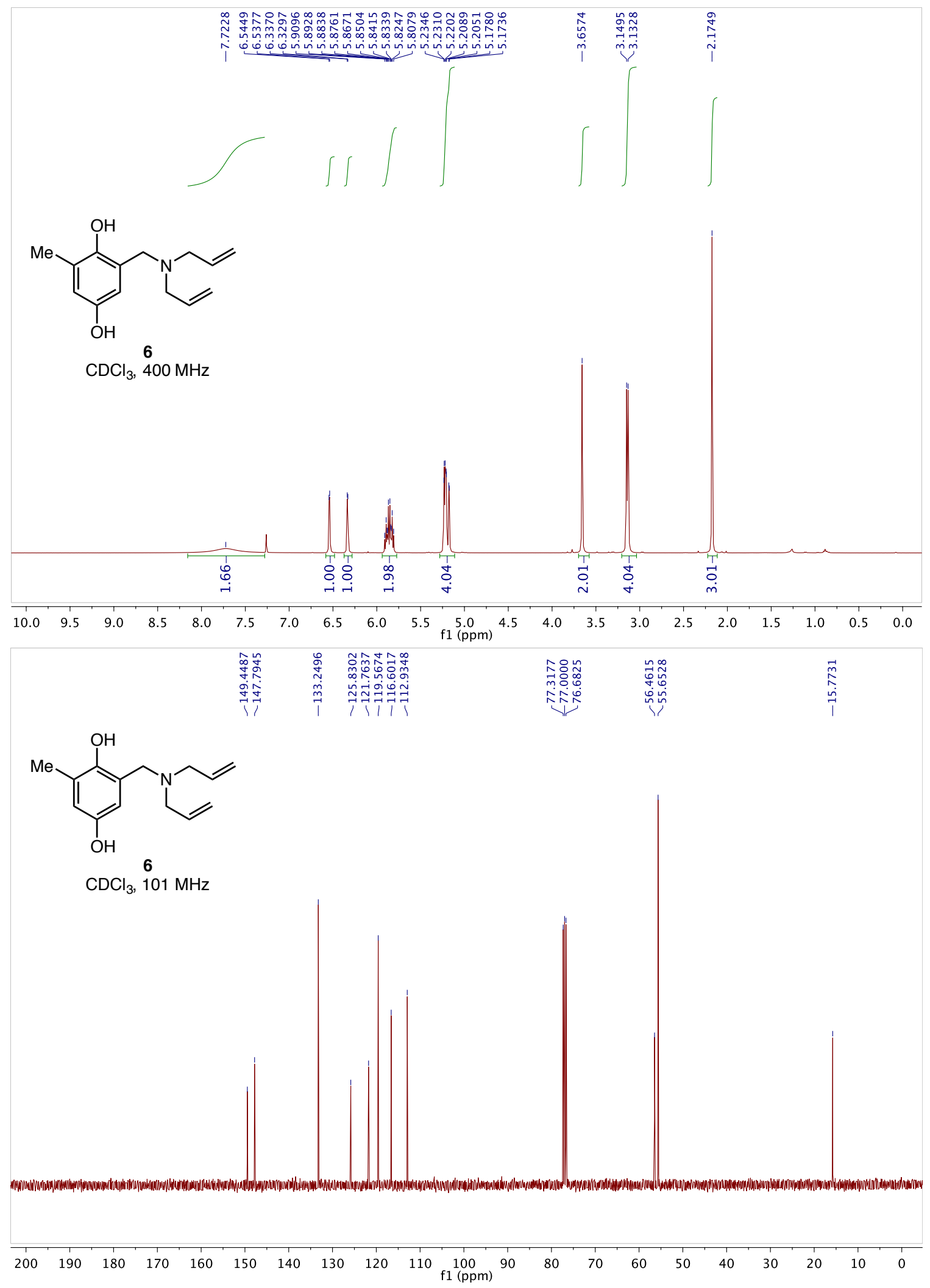



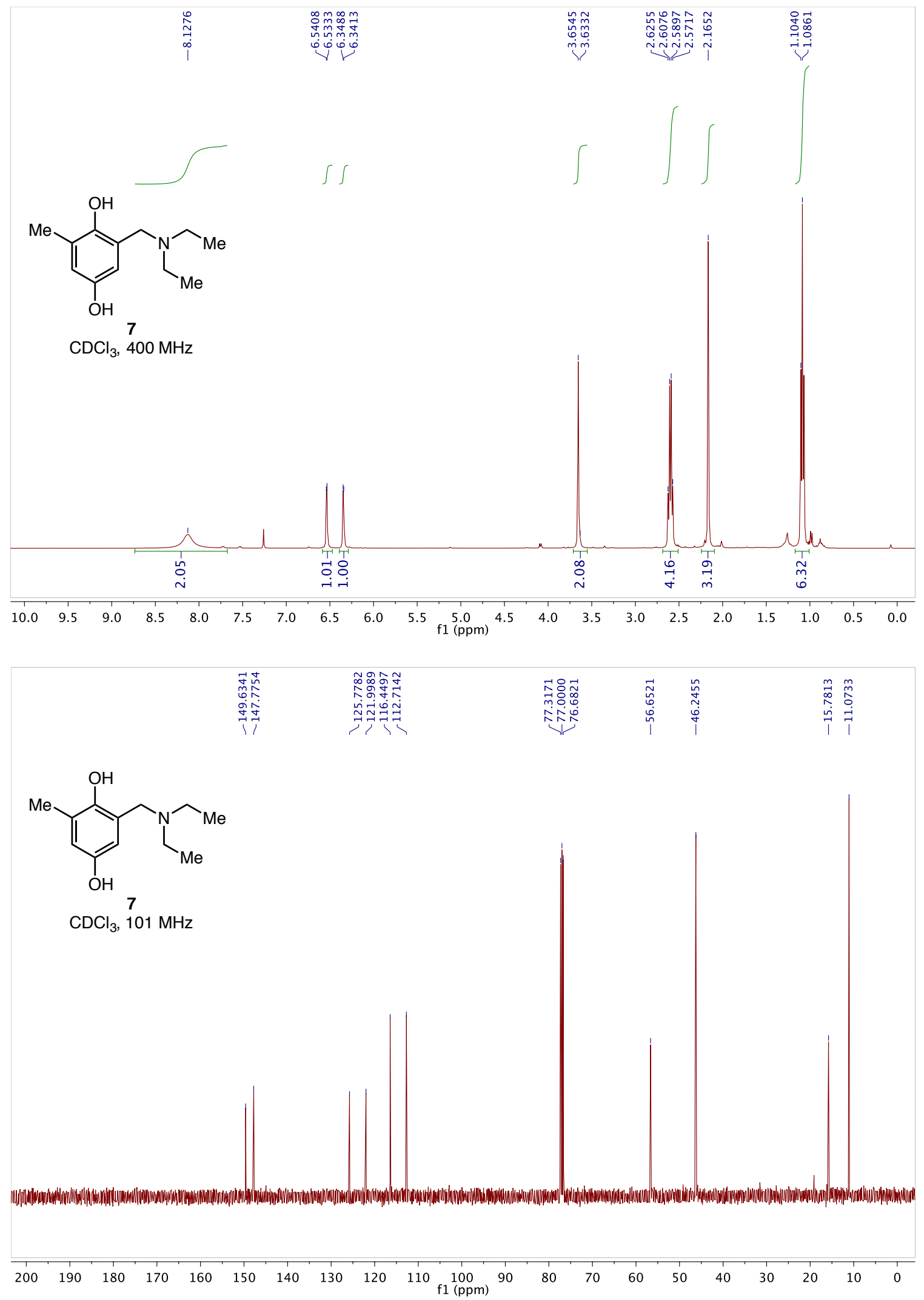

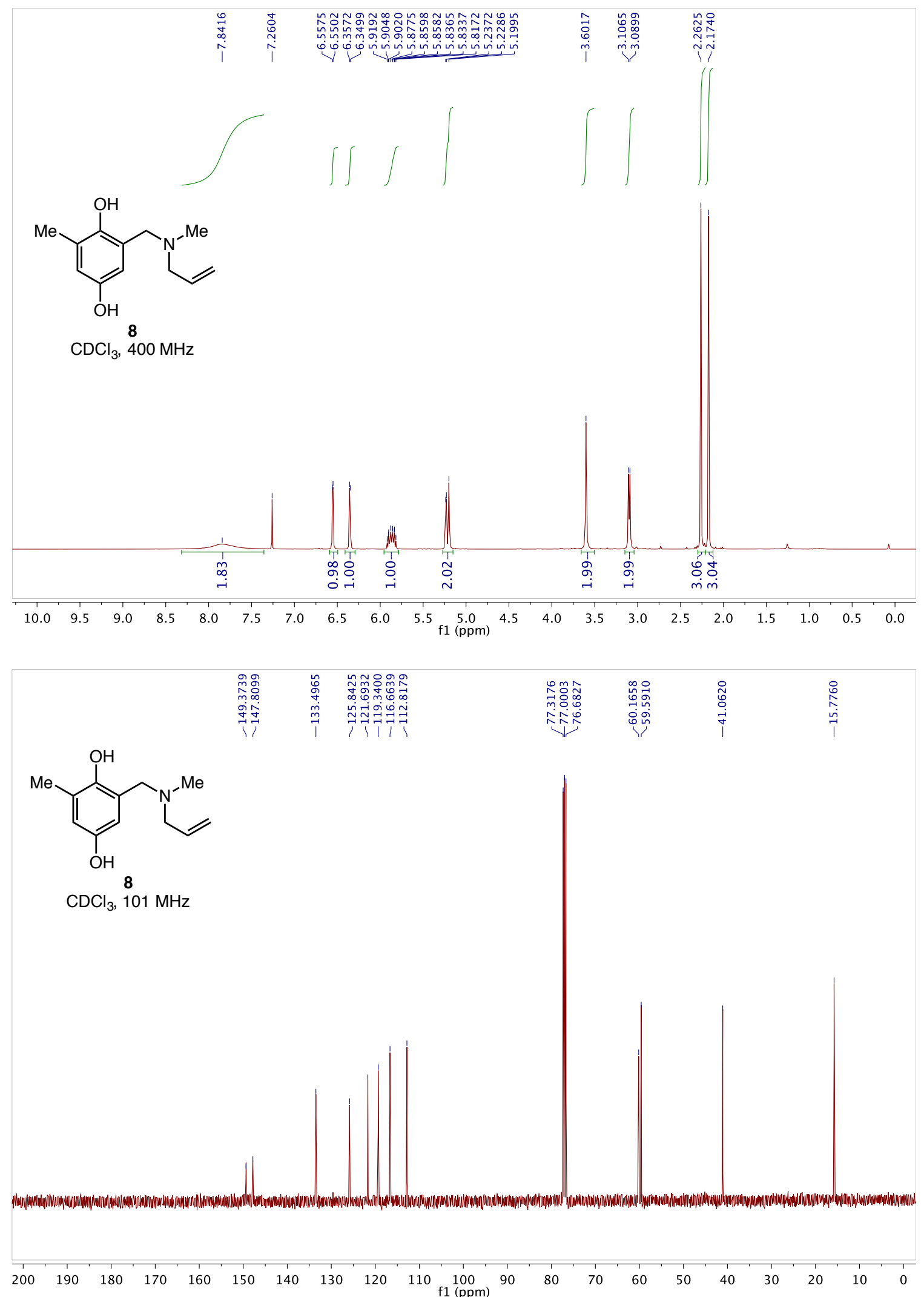


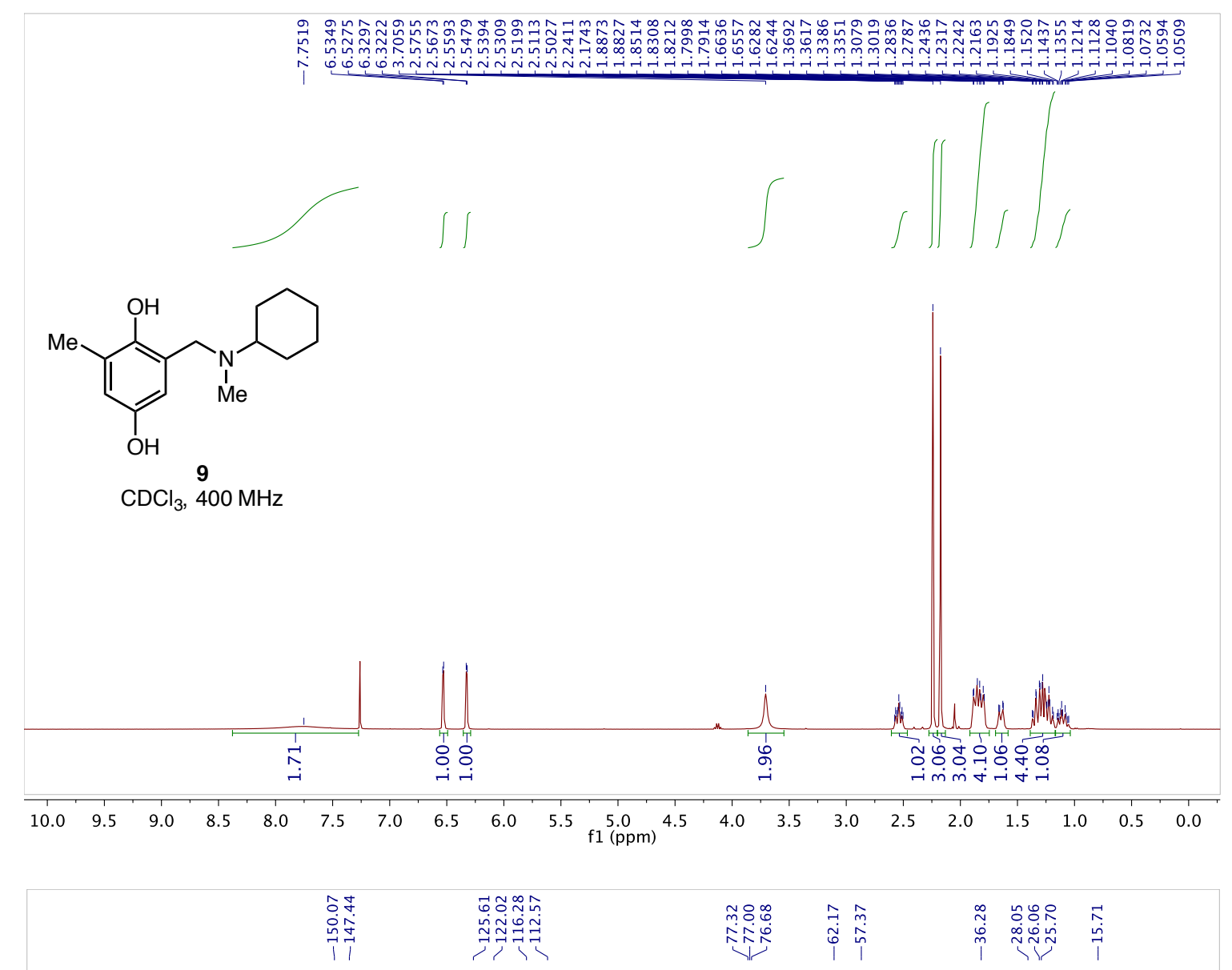<smiles>Cc1cc(O)cc(CN(C)C2CCCCC2)c1O</smiles>

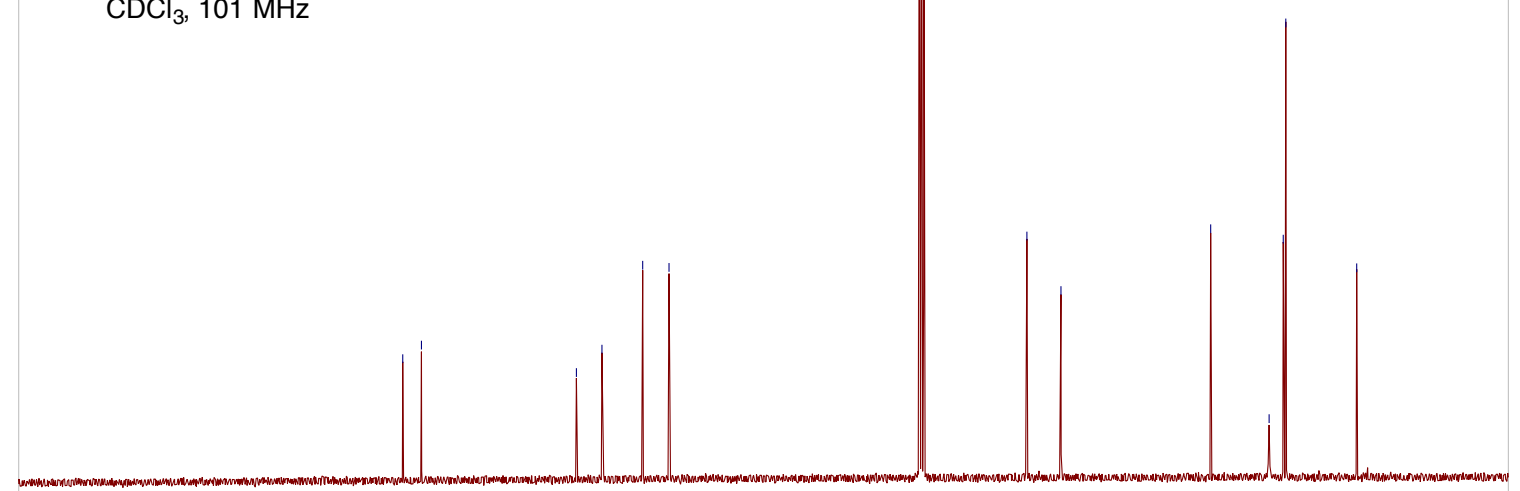

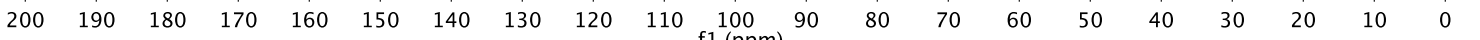



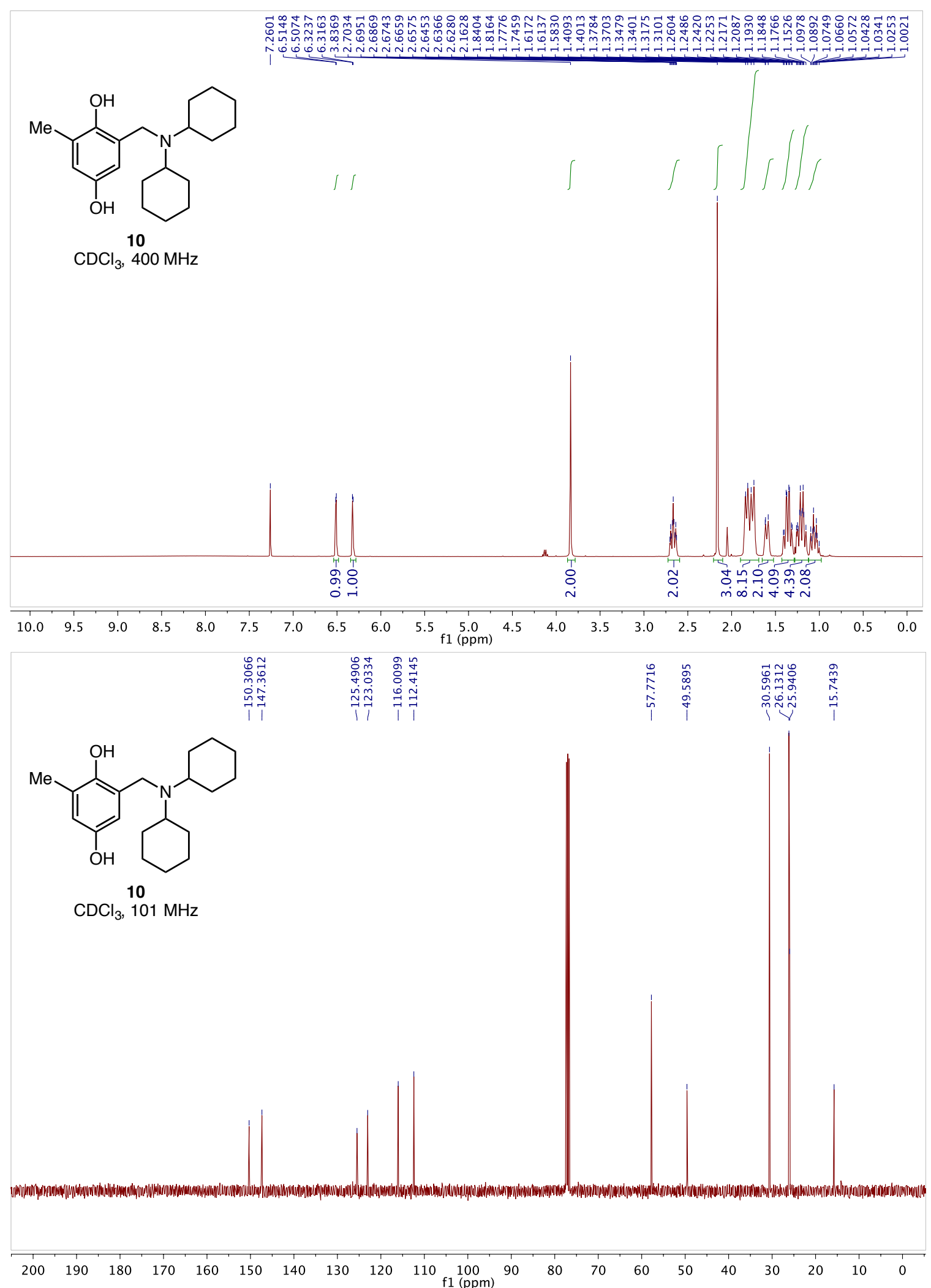

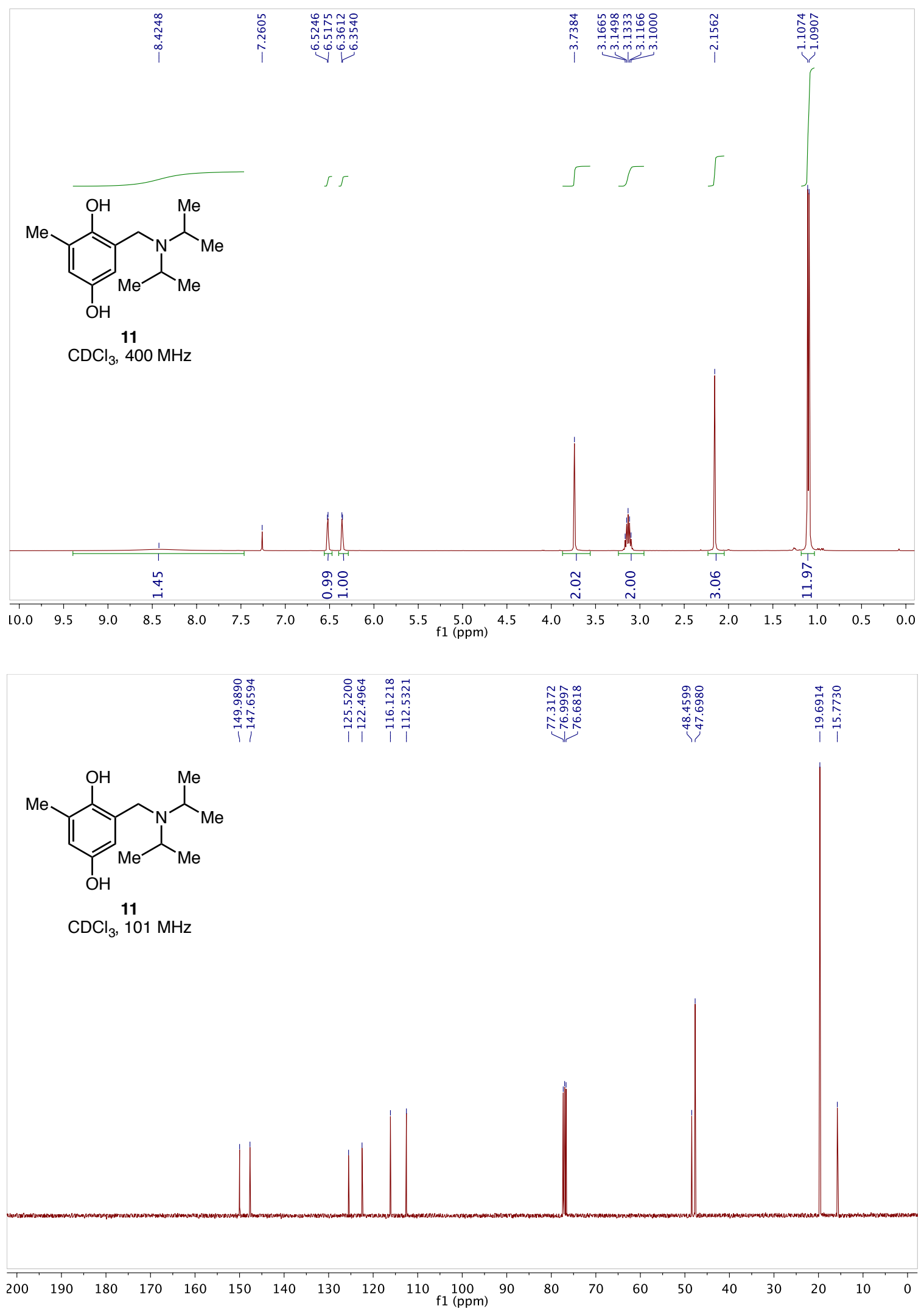


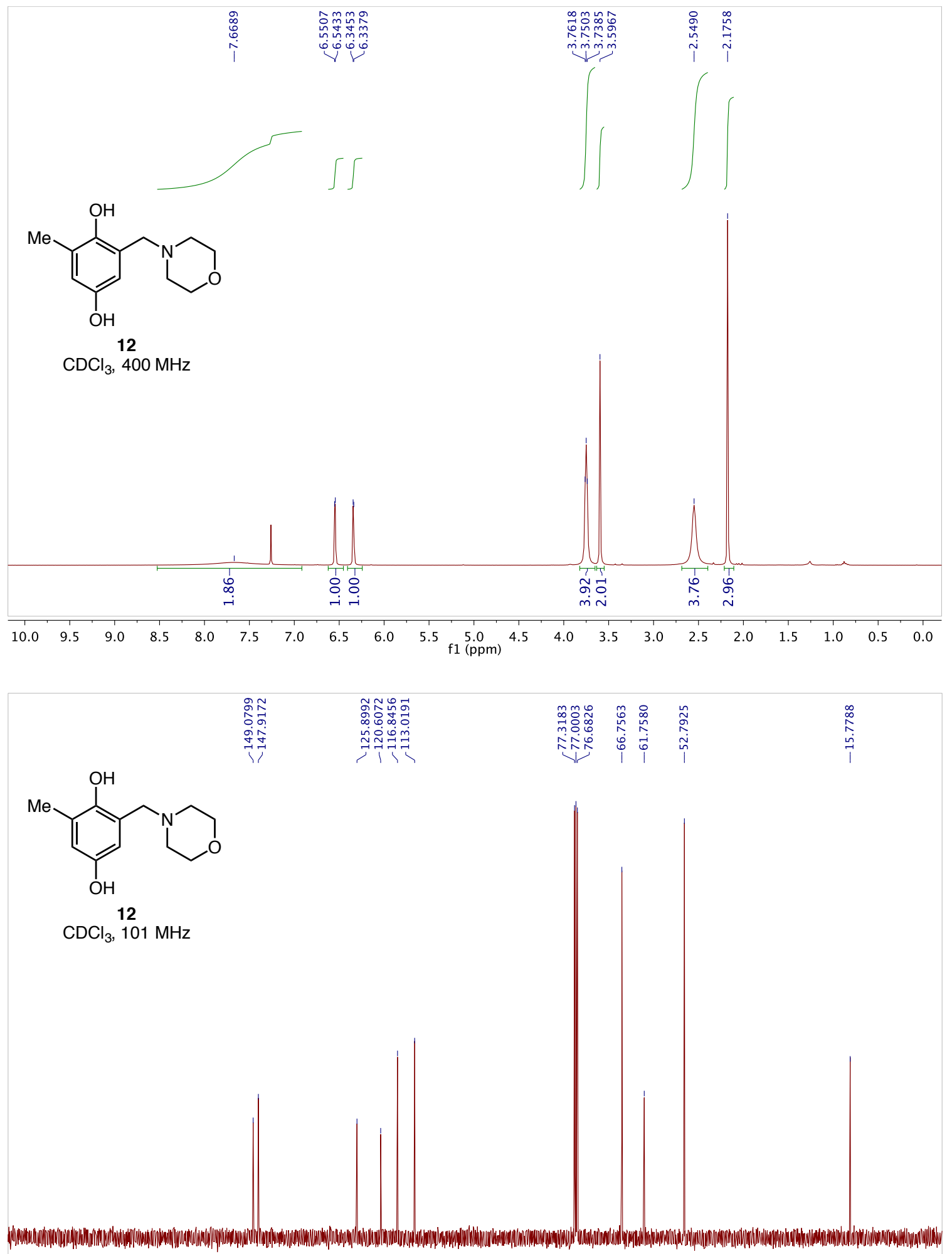

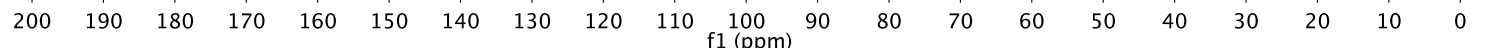




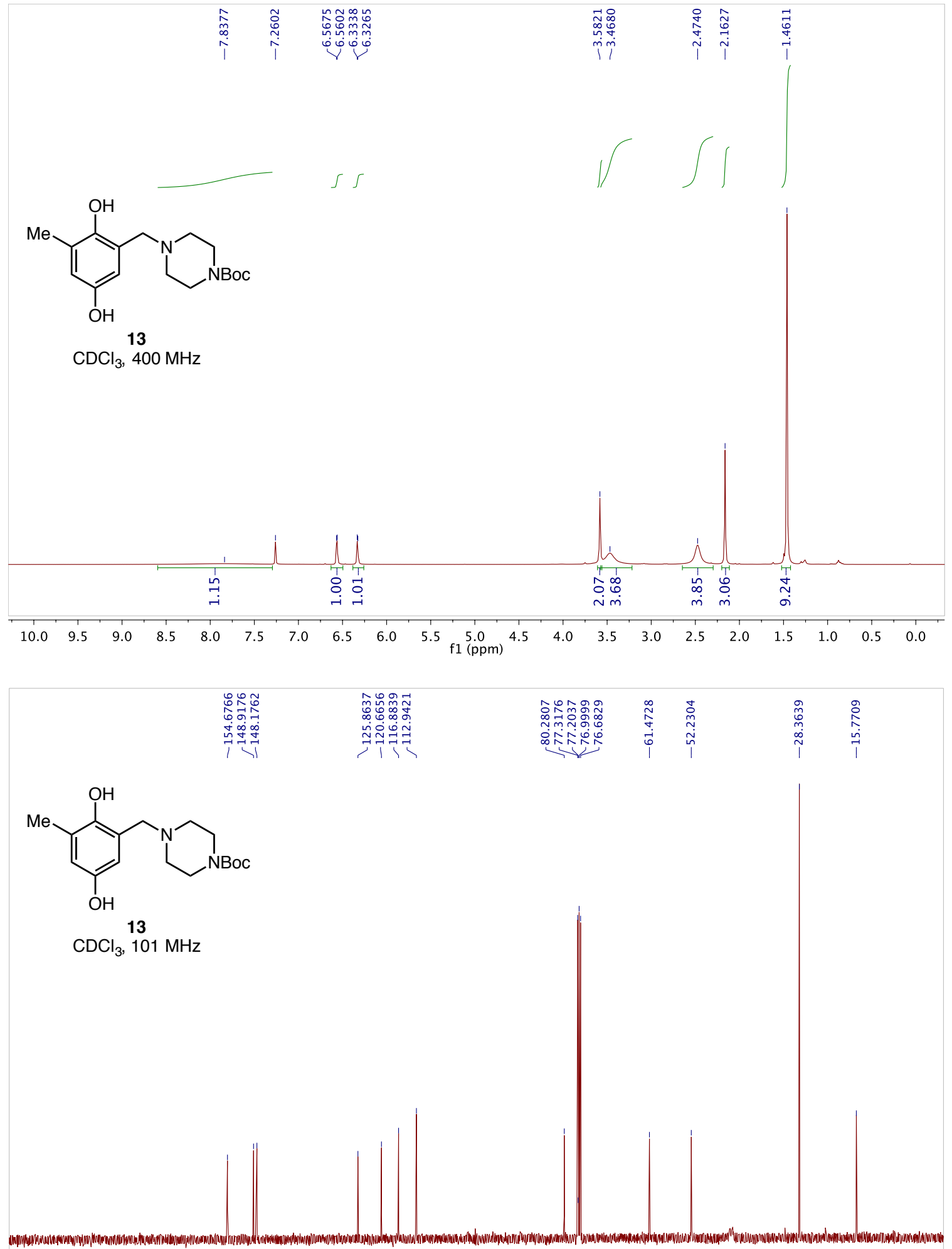

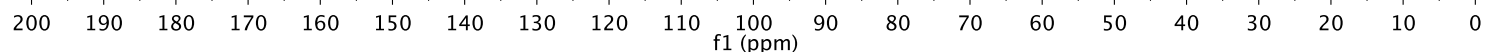



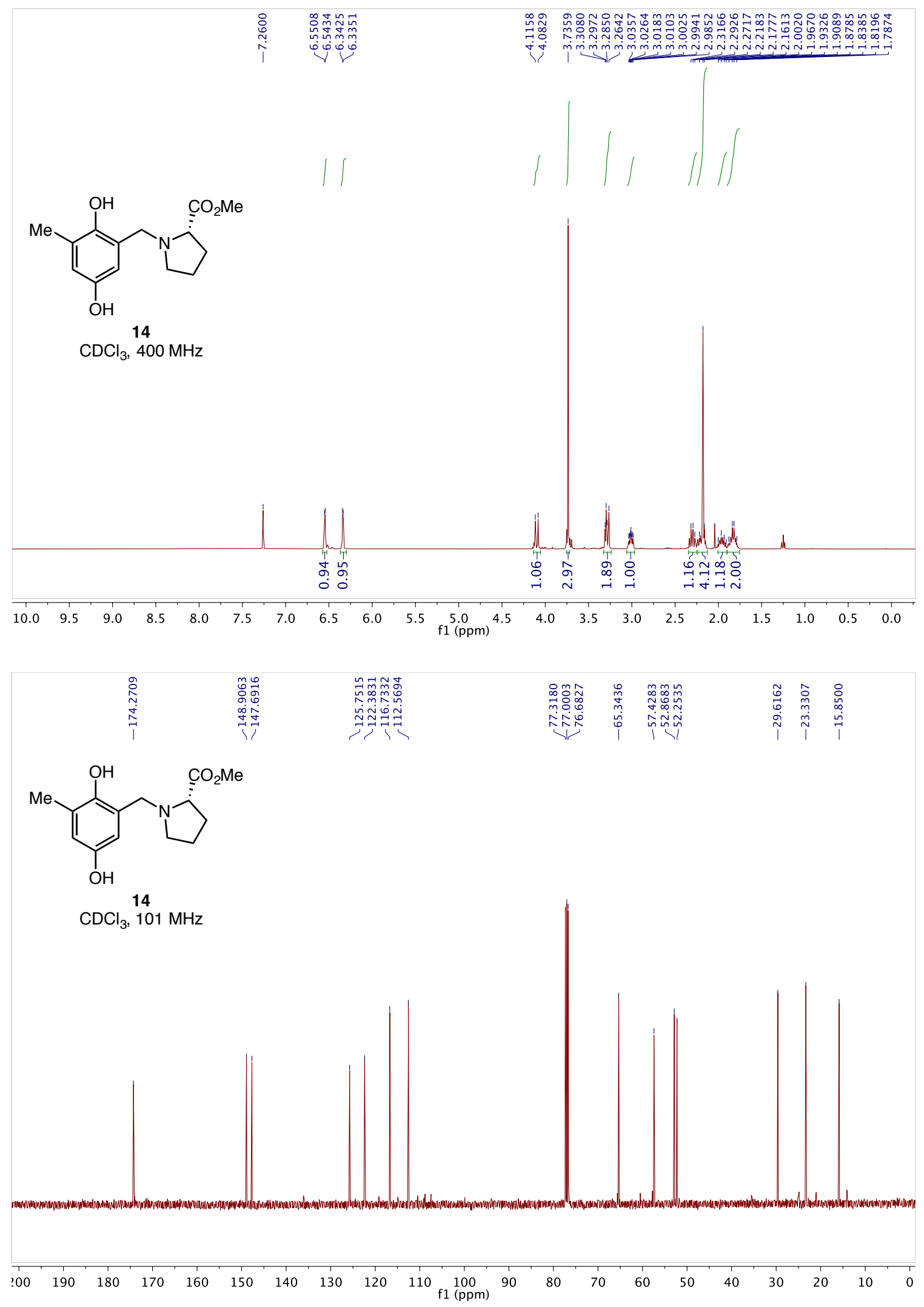


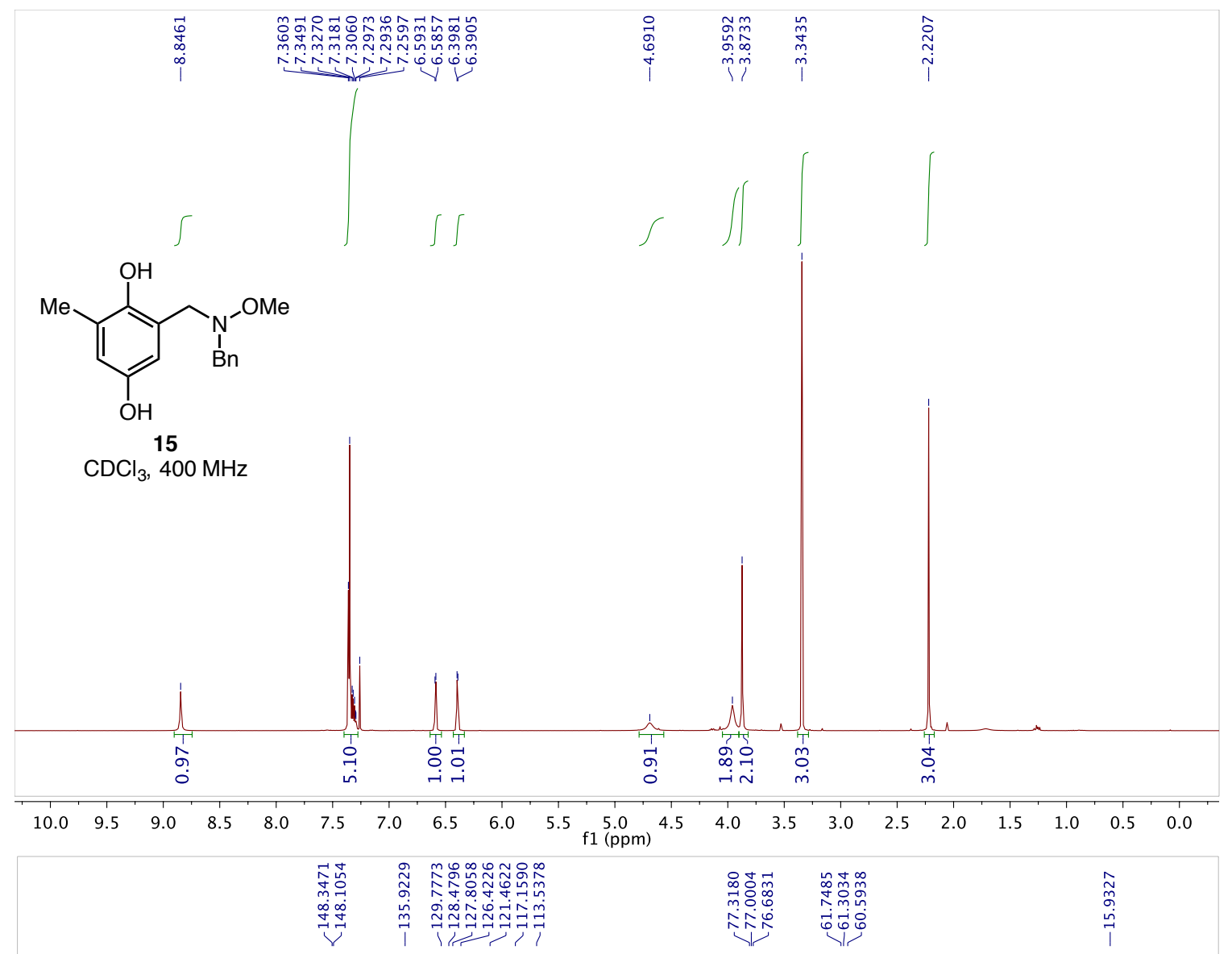<smiles>COc1cc(O)cc(CN(Cc2ccccc2)OC)c1O</smiles>

15

$\mathrm{CDCl}_{3}, 101 \mathrm{MHz}$
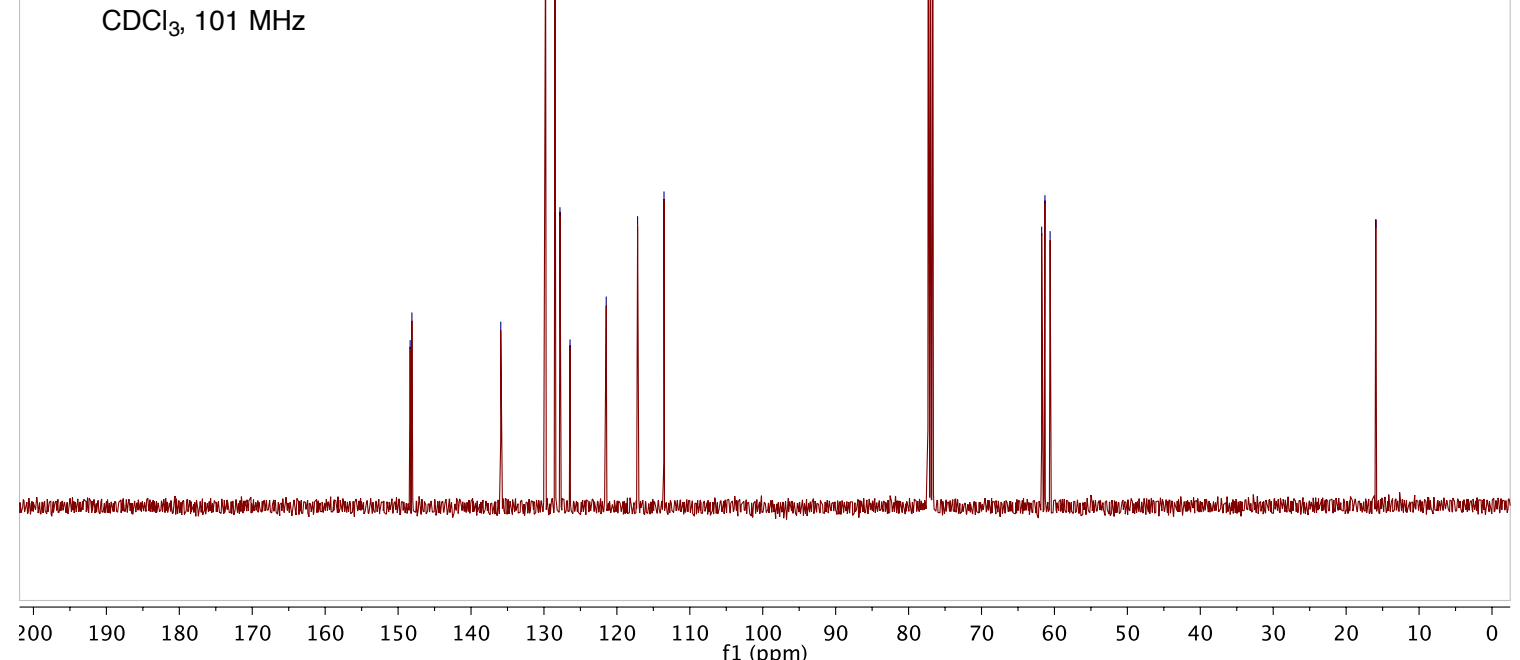


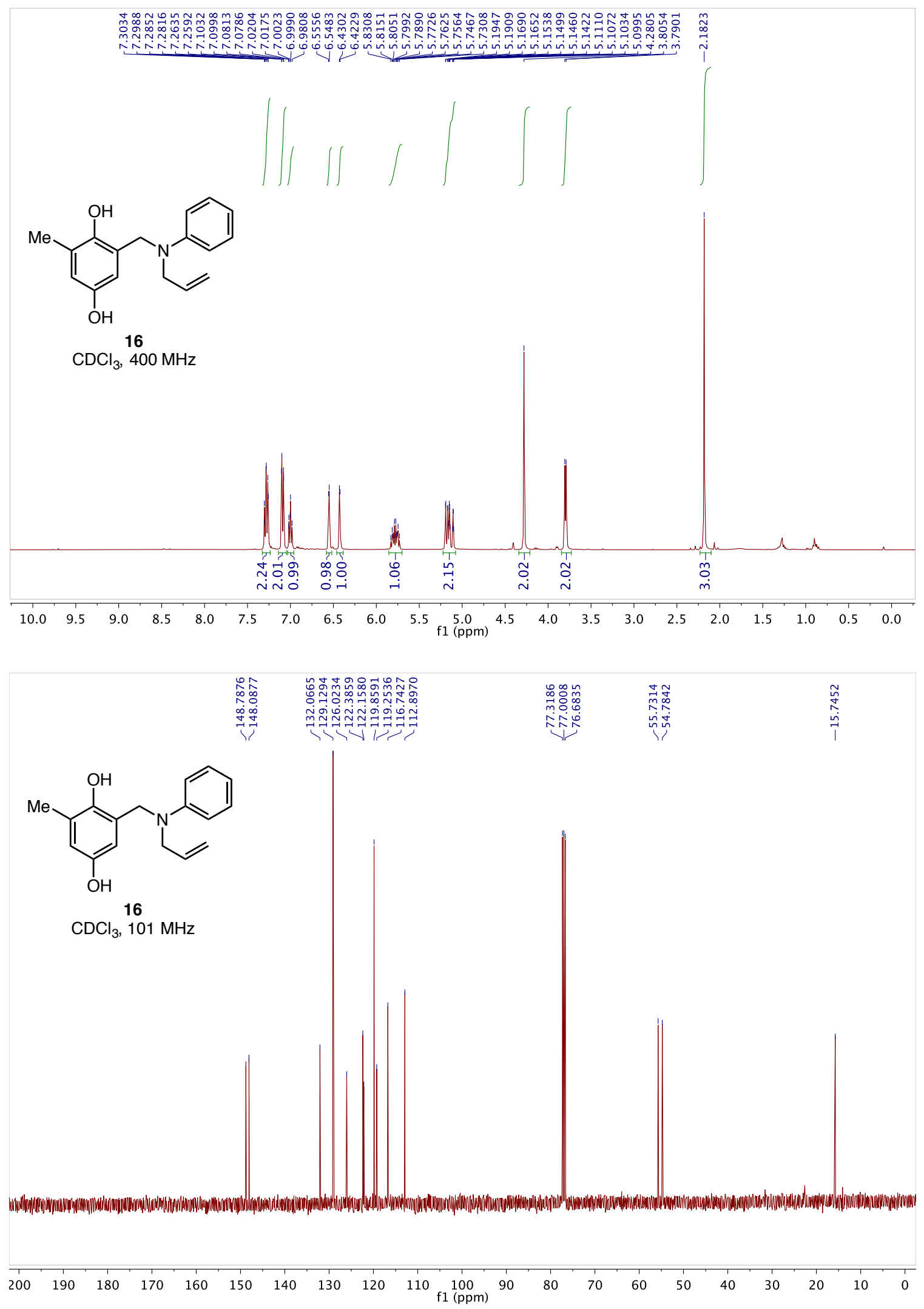




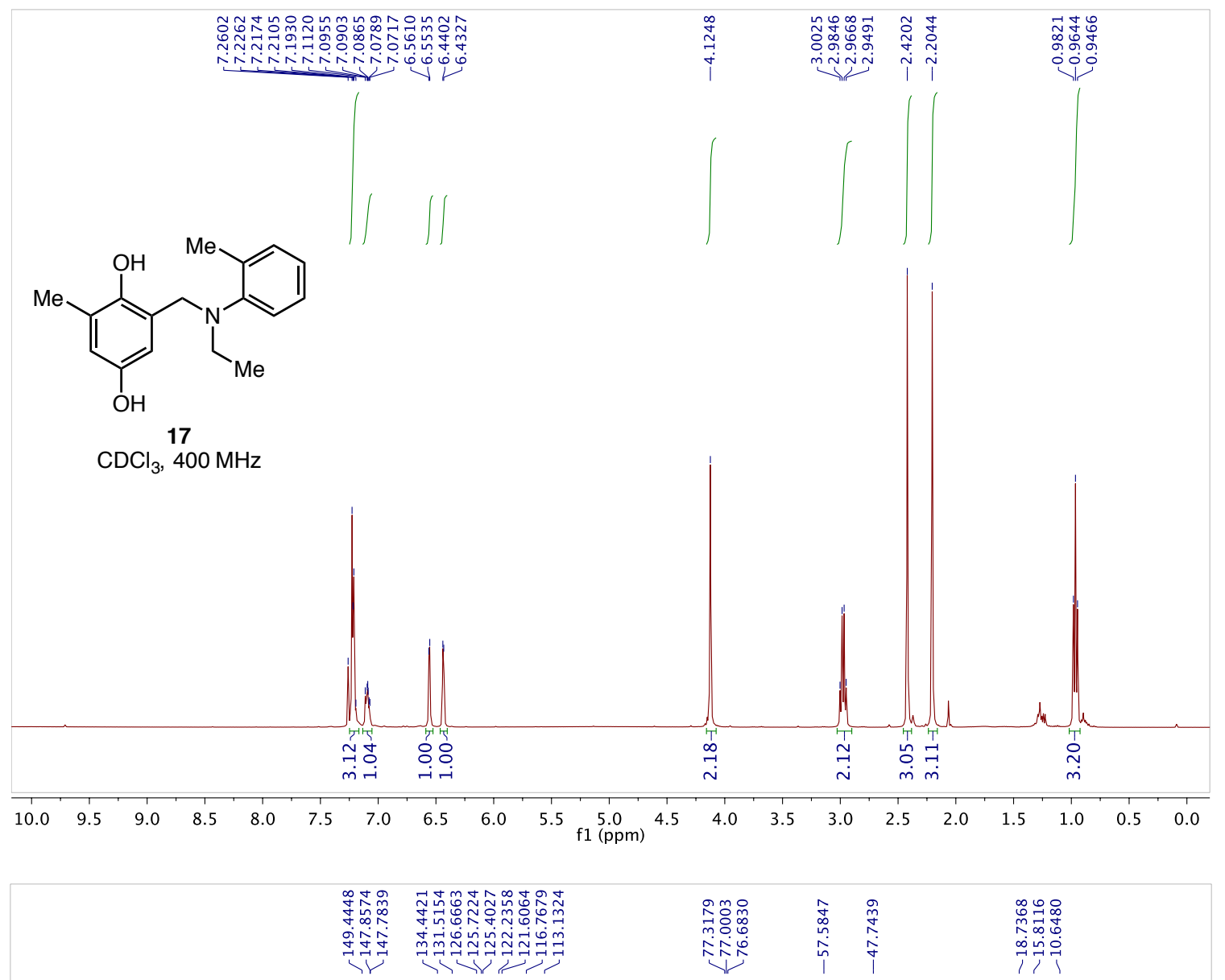<smiles>Cc1ccccc1N(Cc1cc(O)cc(C)c1O)C[N+](=O)[O-]</smiles>

$\mathrm{CDCl}_{3}, 1701 \mathrm{MHz}$

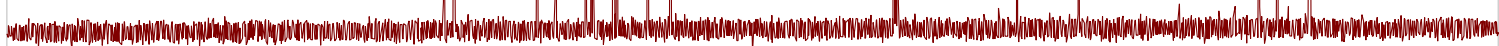

$\begin{array}{lllllllllllllllllllllllll}210 & 200 & 190 & 180 & 170 & 160 & 150 & 140 & 130 & 120 & 110 \underset{\mathrm{f} 1}{100}(\mathrm{ppm}) & 90 & 80 & 70 & 60 & 50 & 40 & 30 & 20 & 10 & 0 & -10\end{array}$ 

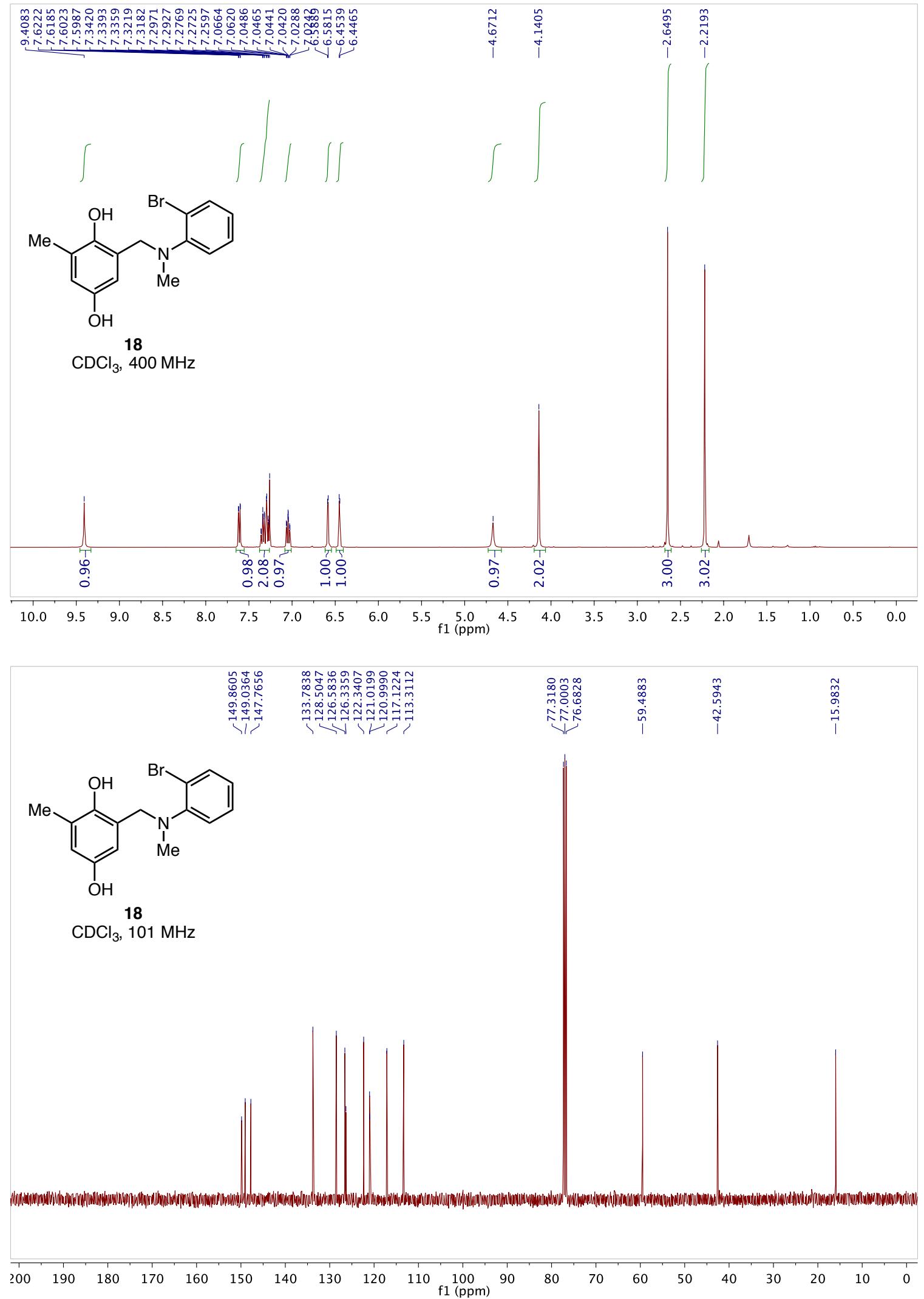

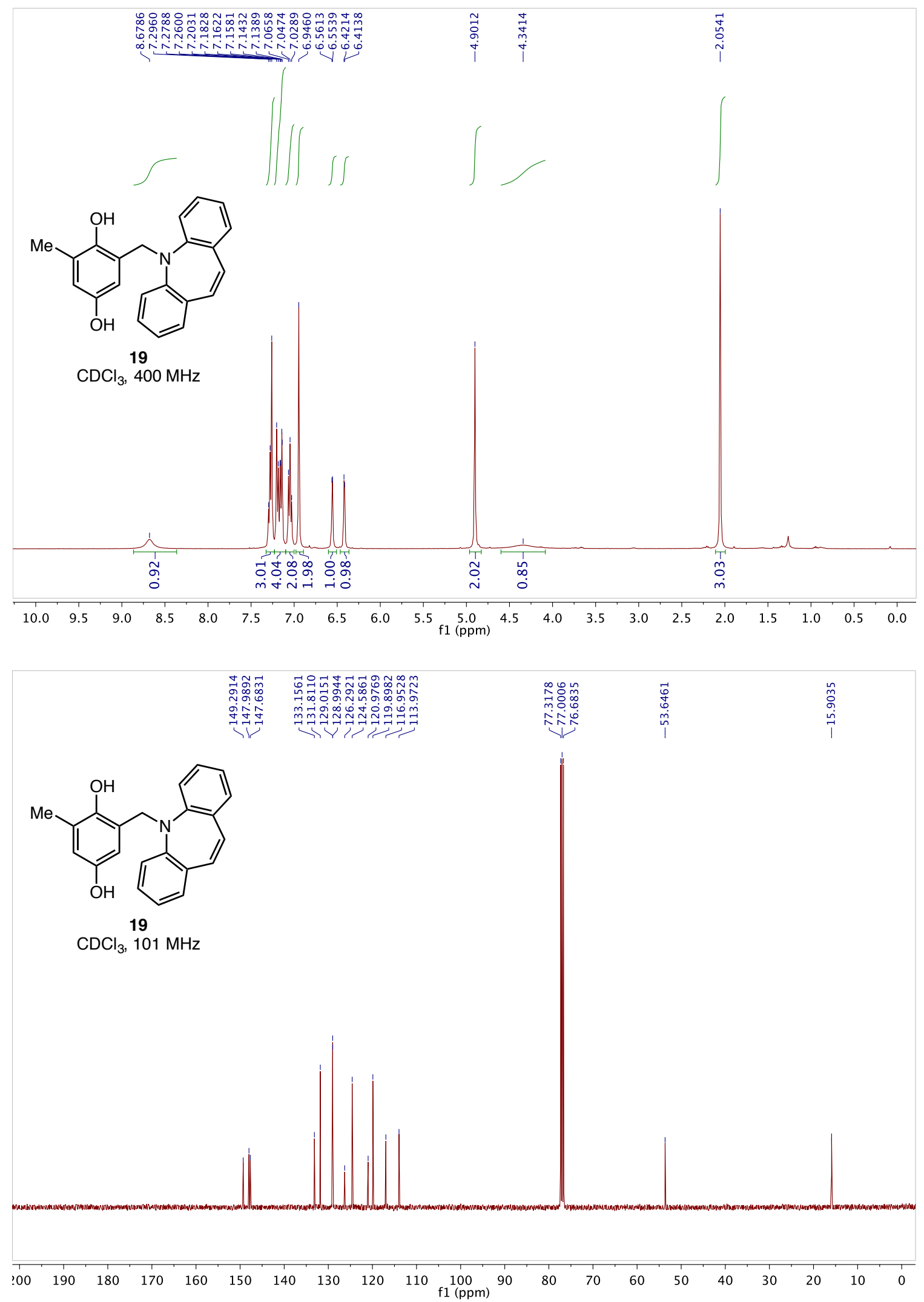

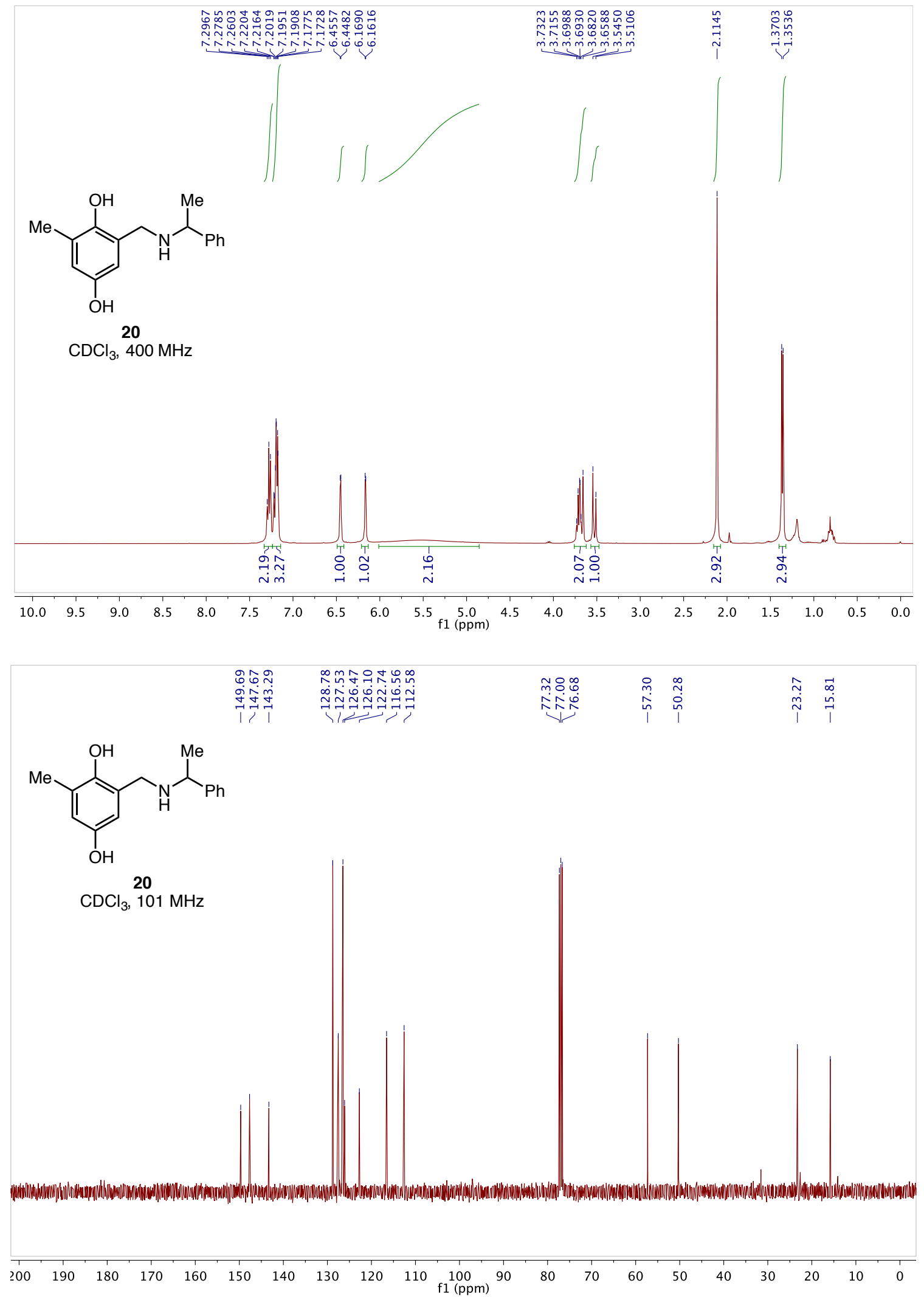

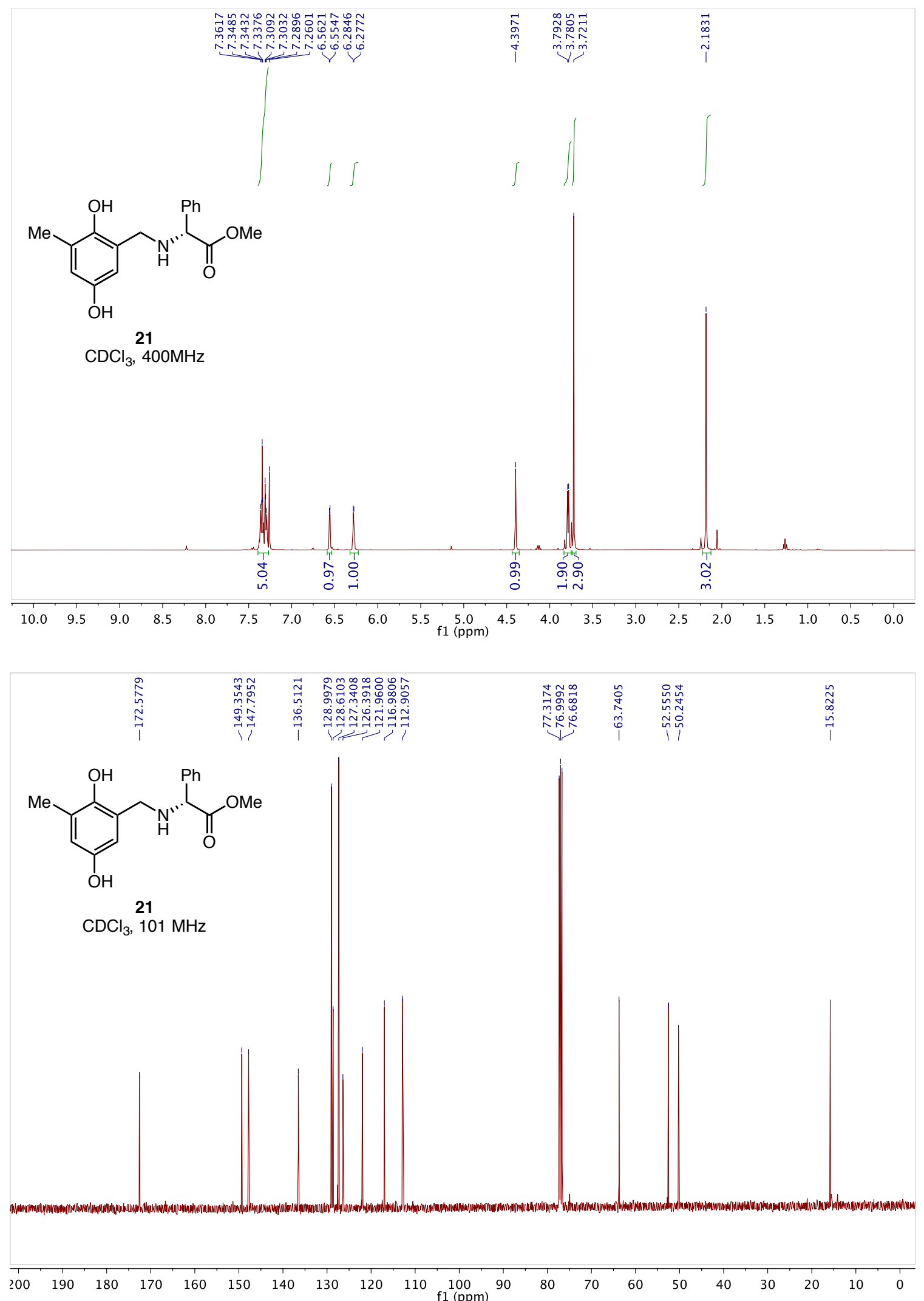

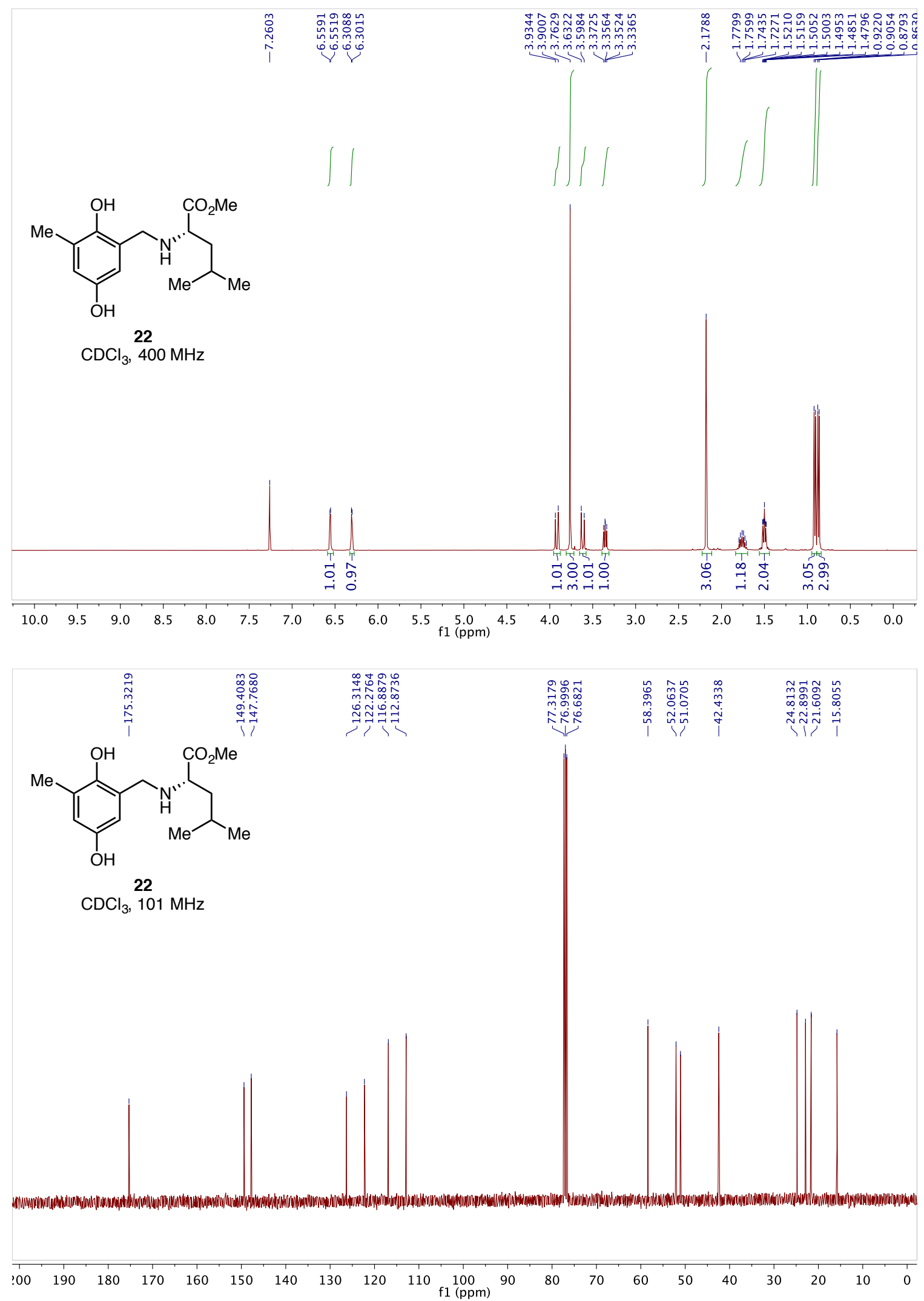

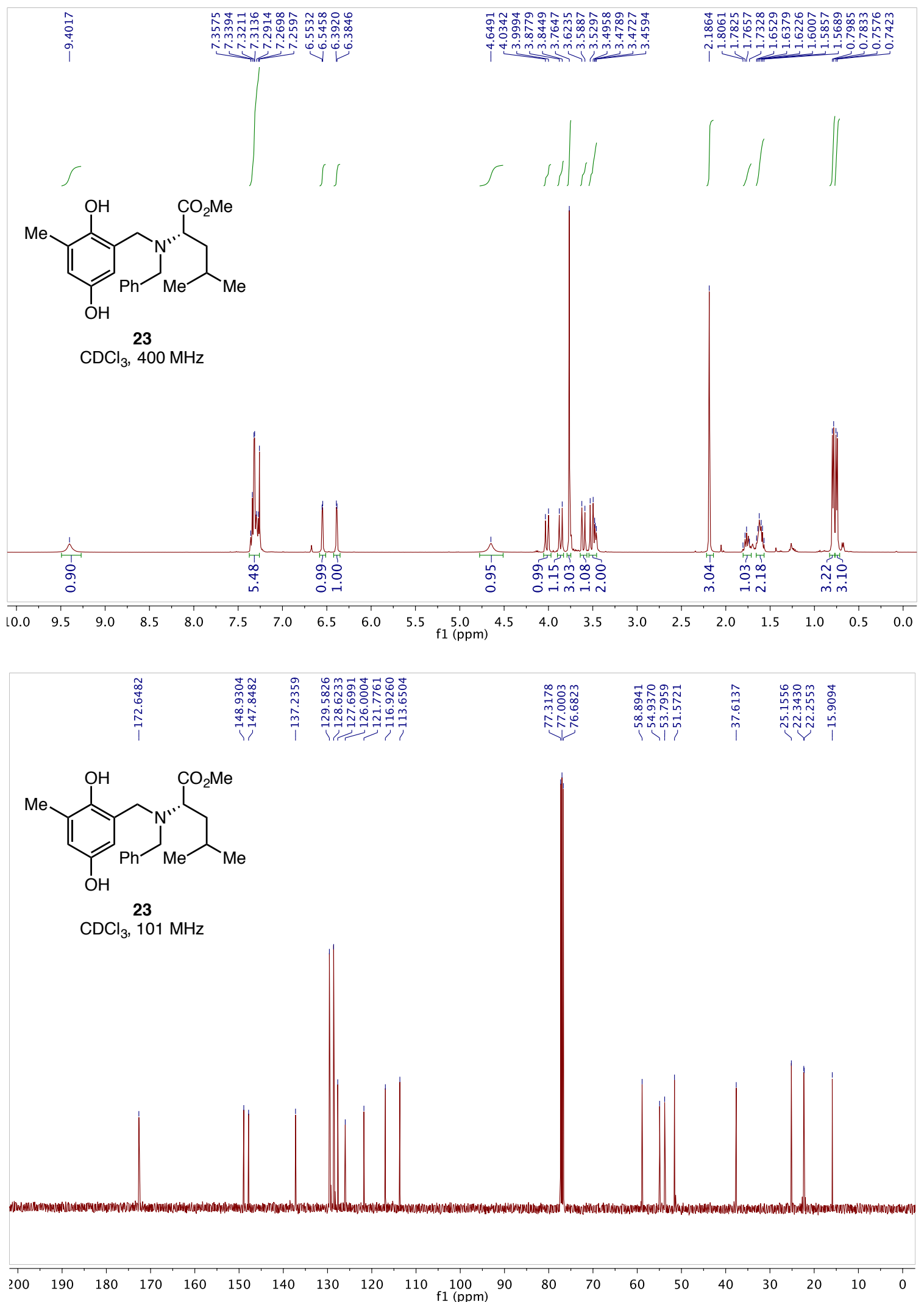


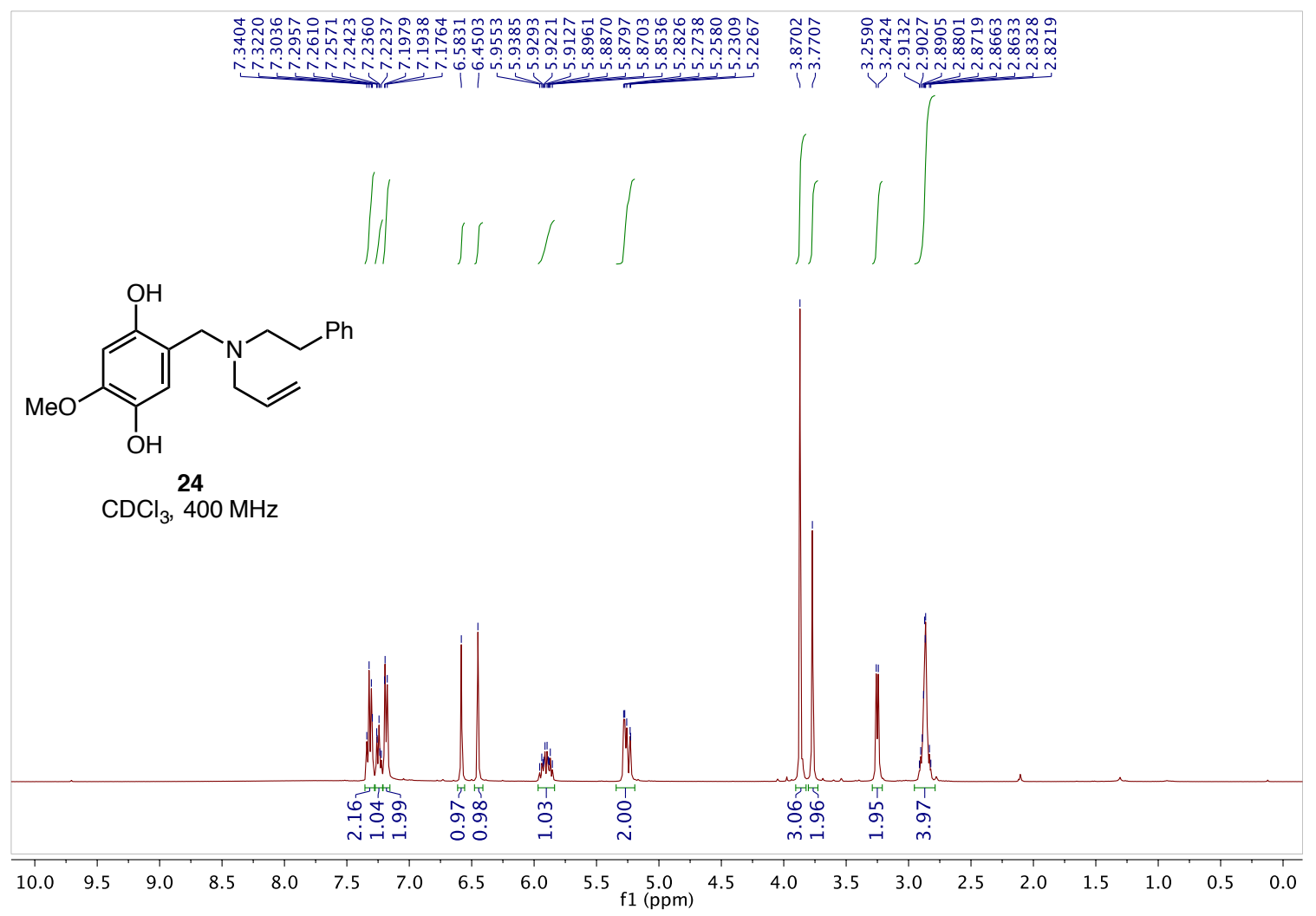

\begin{tabular}{|c|c|c|}
\hline 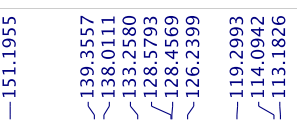 & $\stackrel{0}{1}$ & 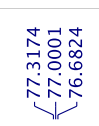 \\
\hline
\end{tabular}<smiles>C=CCN(CCc1ccccc1)Cc1cc(O)c(OC)cc1O</smiles>

24

$\mathrm{CDCl}_{3}, 101 \mathrm{MHz}$

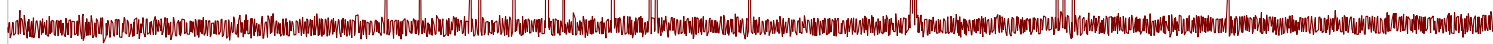

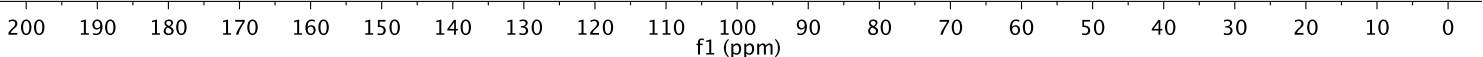




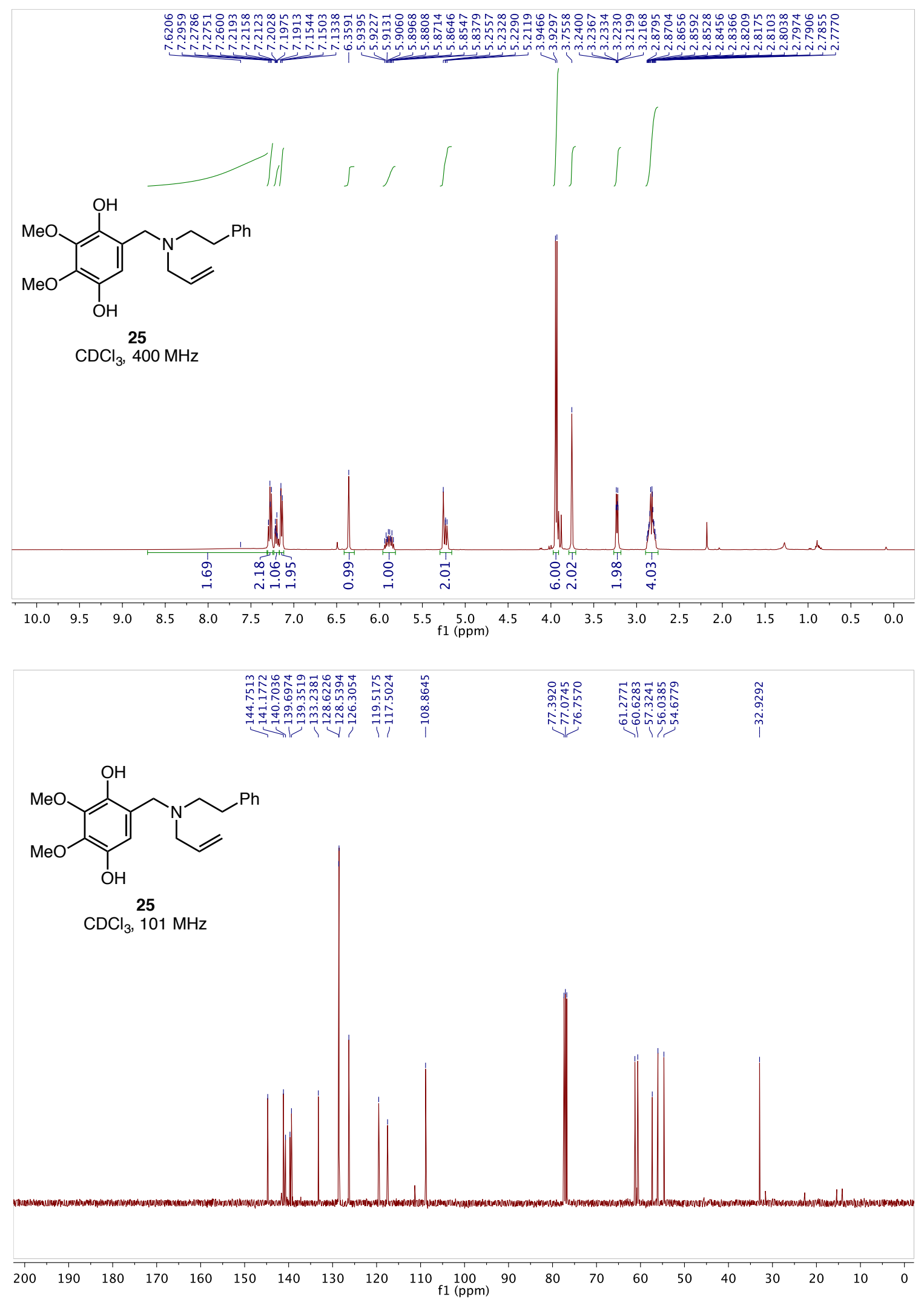



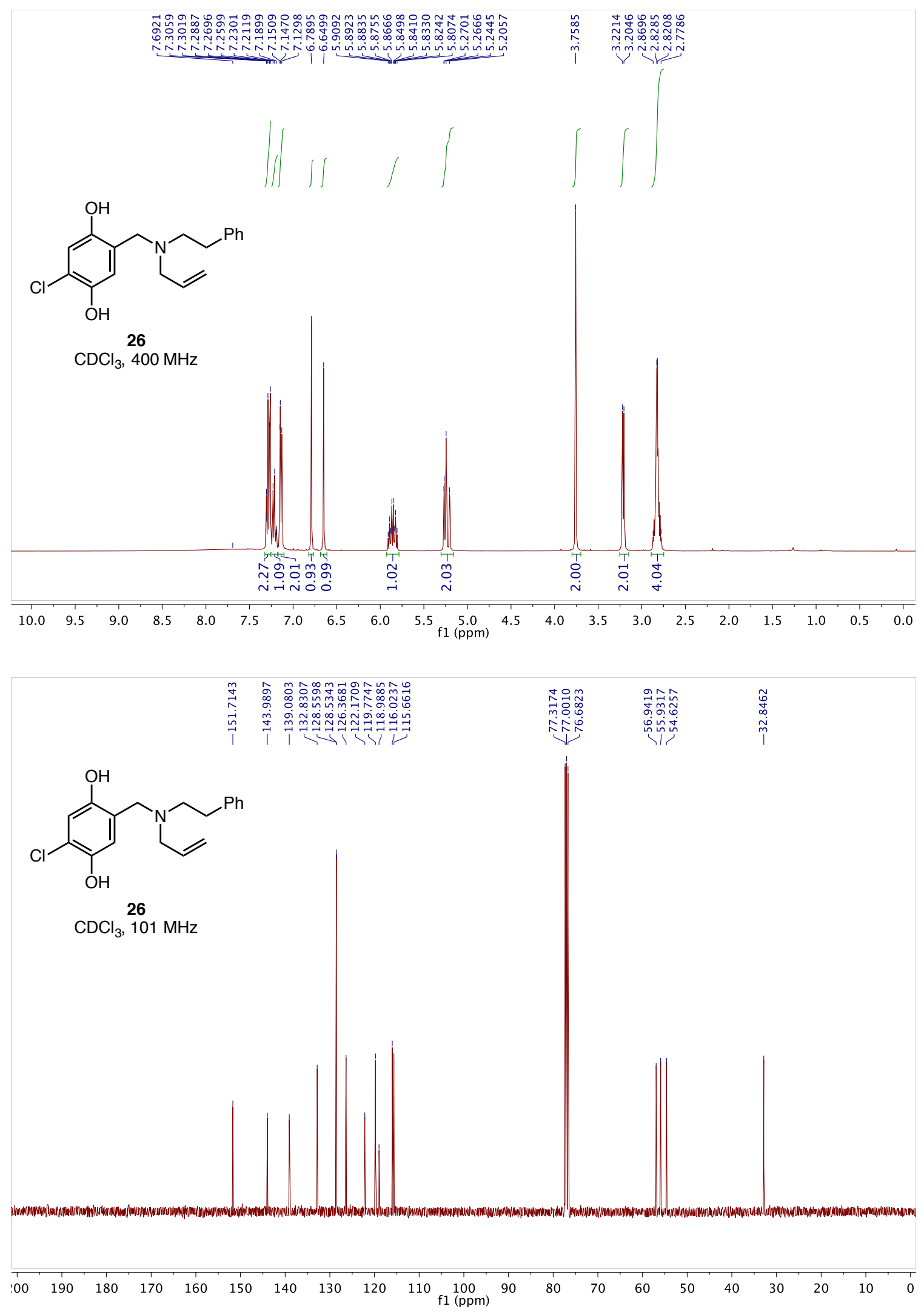

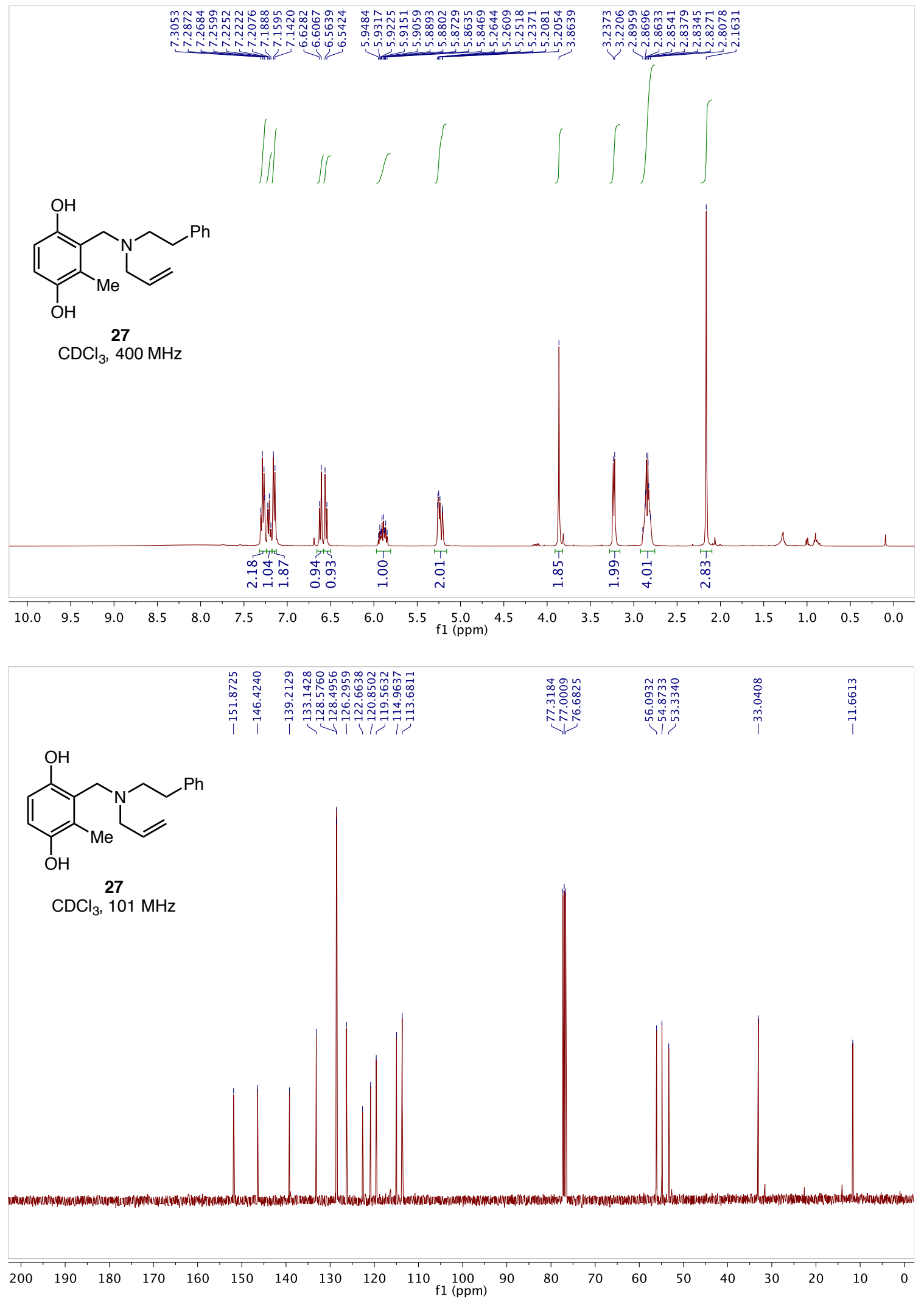

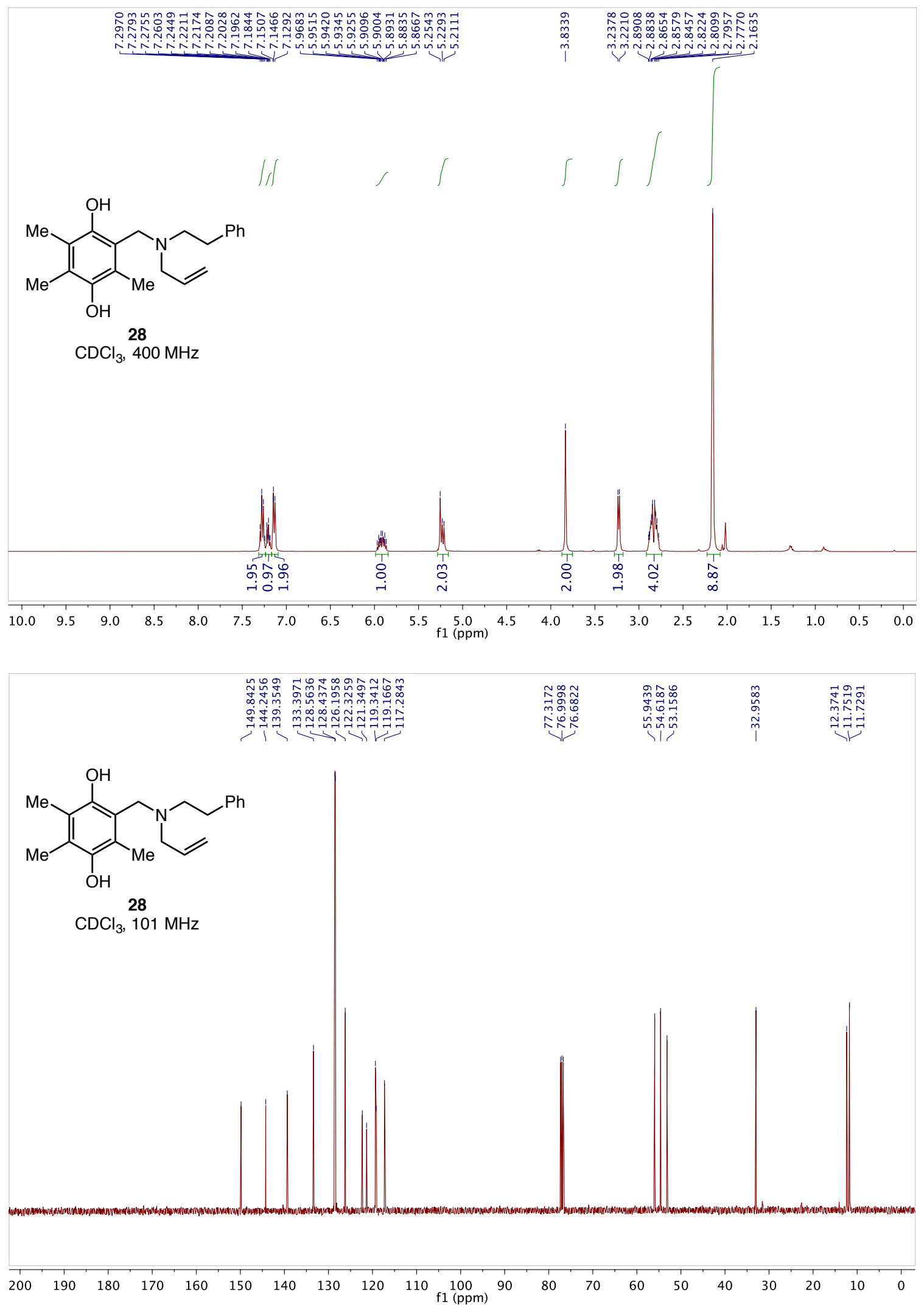

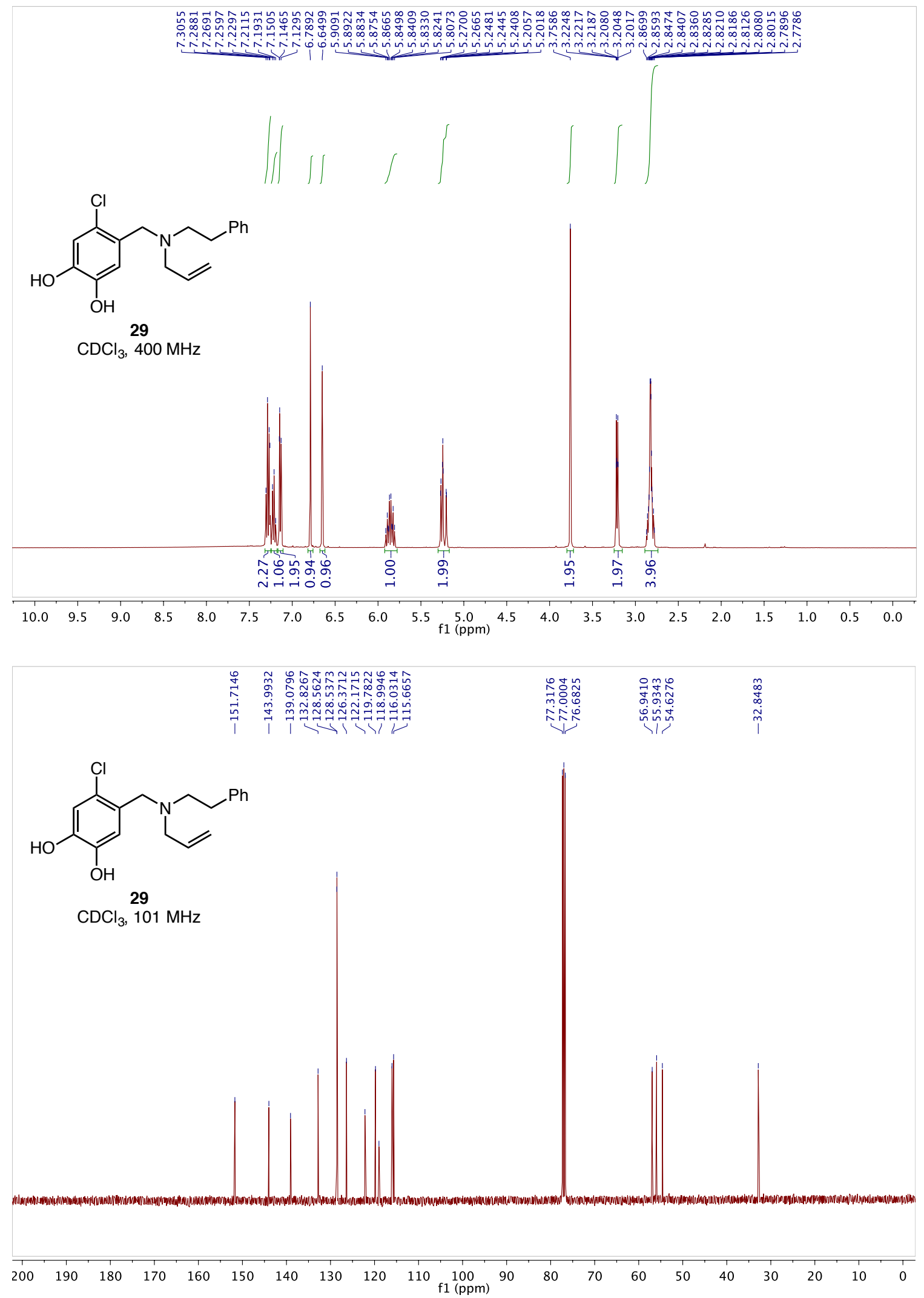

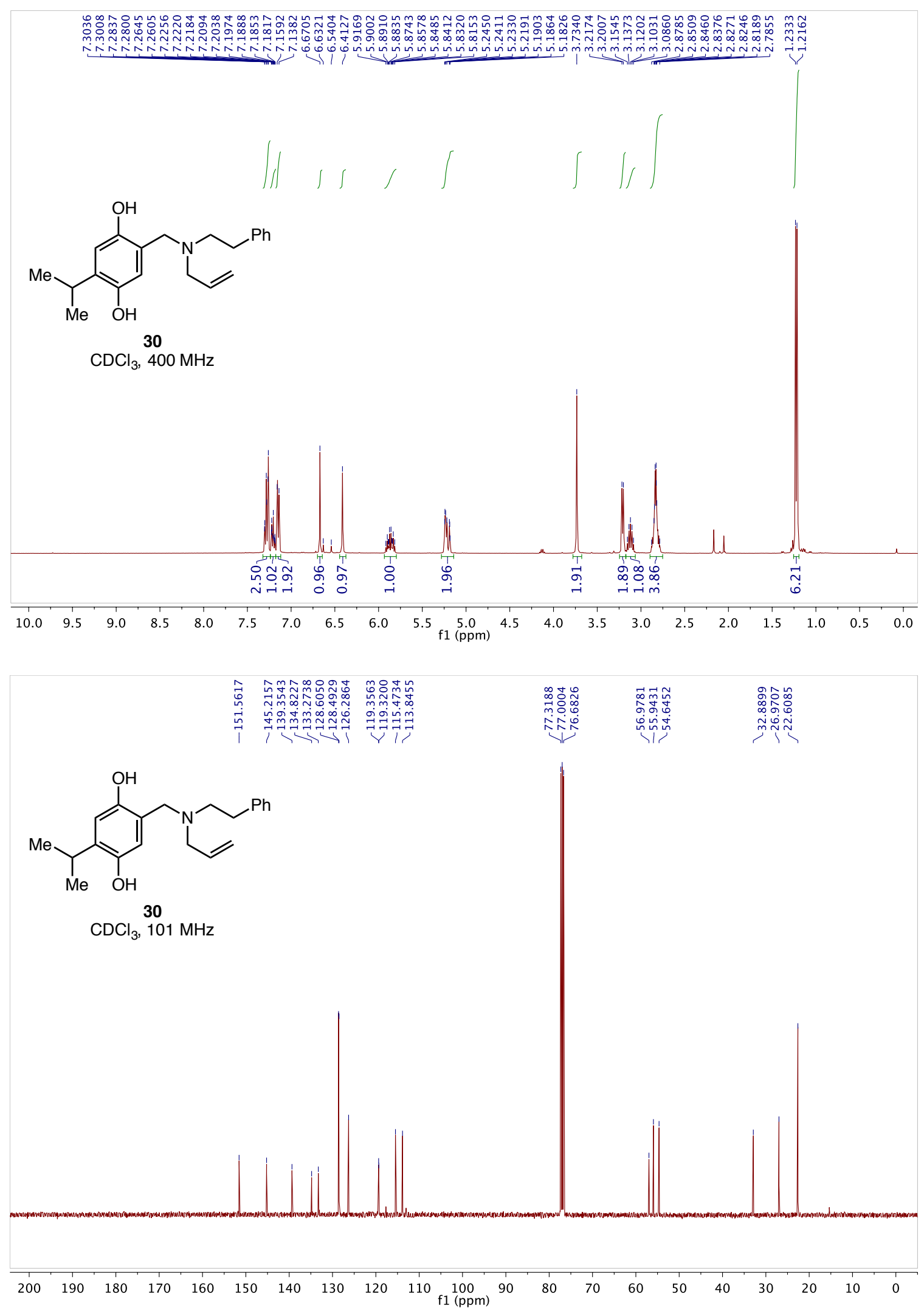


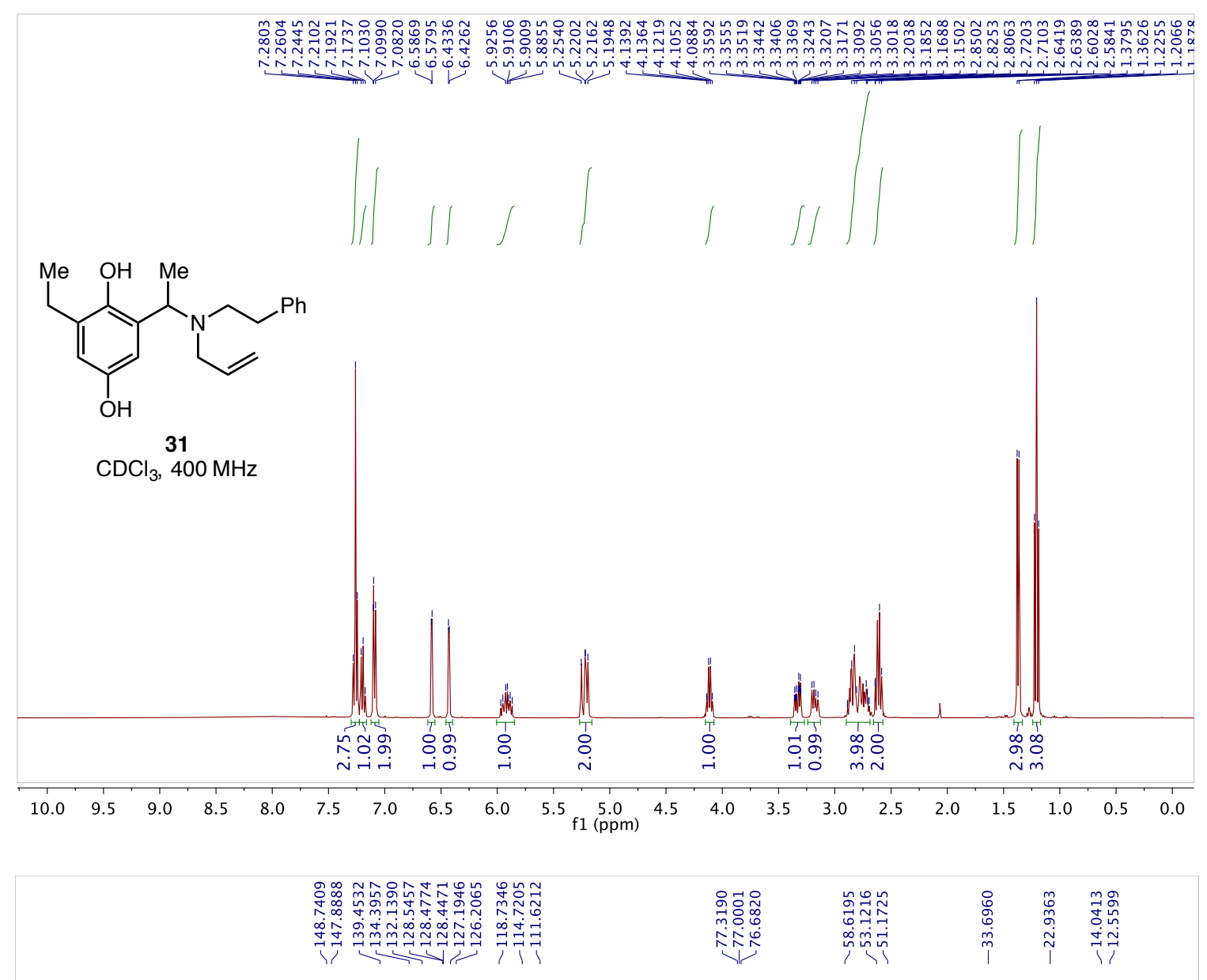<smiles>[CH]Cc1cc(O)cc(C(C)N(CC=C)CCc2ccccc2)c1O</smiles>

31

$\mathrm{CDCl}_{3}, 101 \mathrm{MHz}$

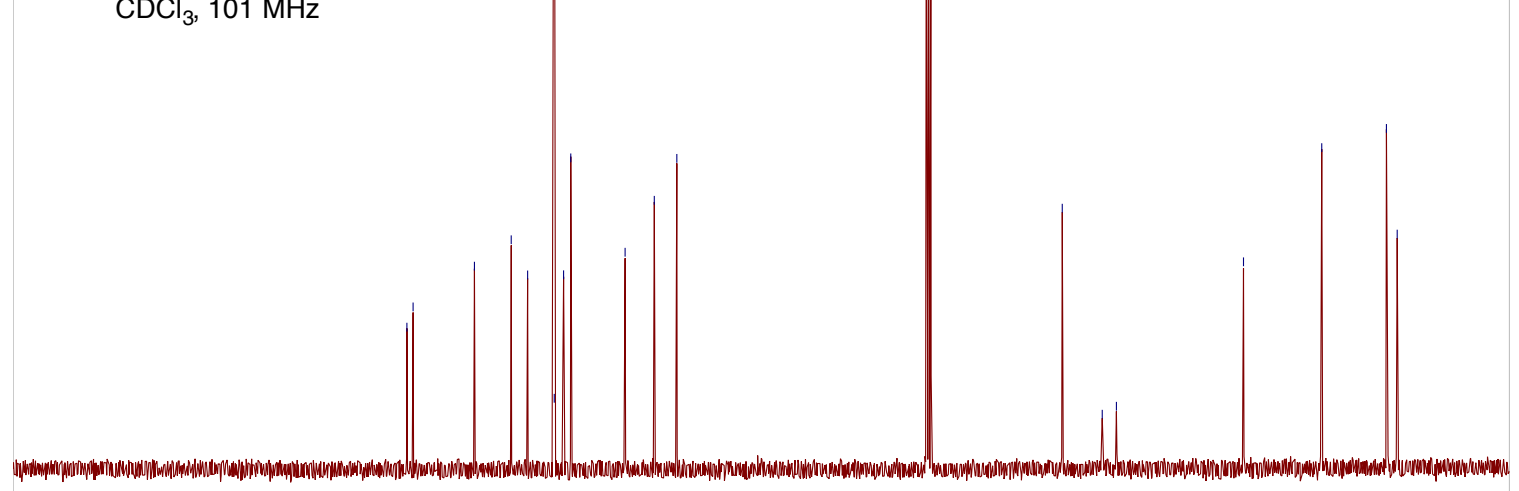

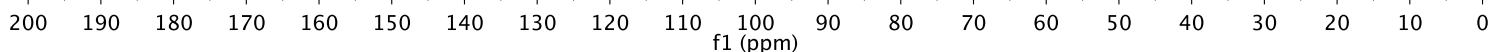




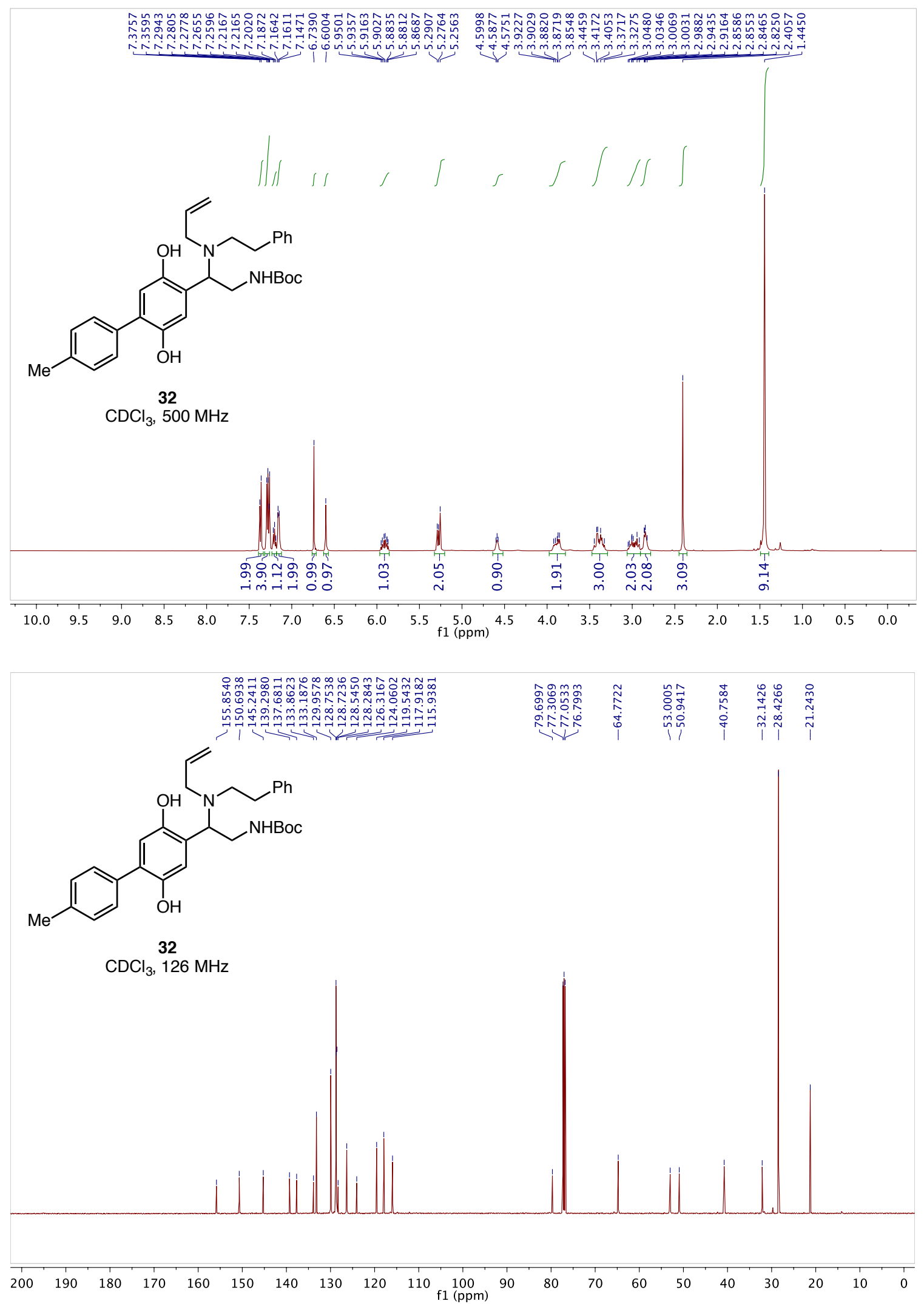



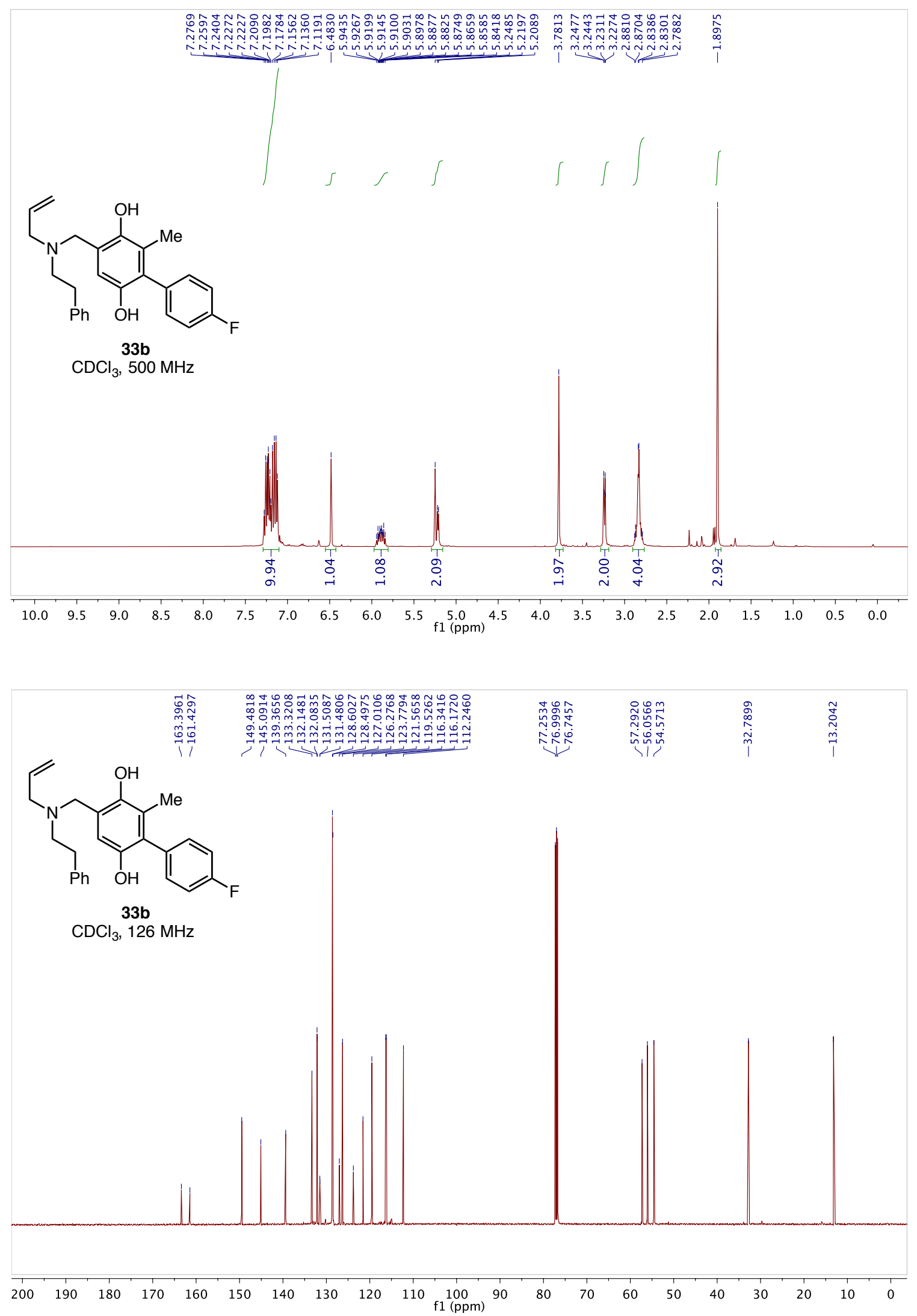


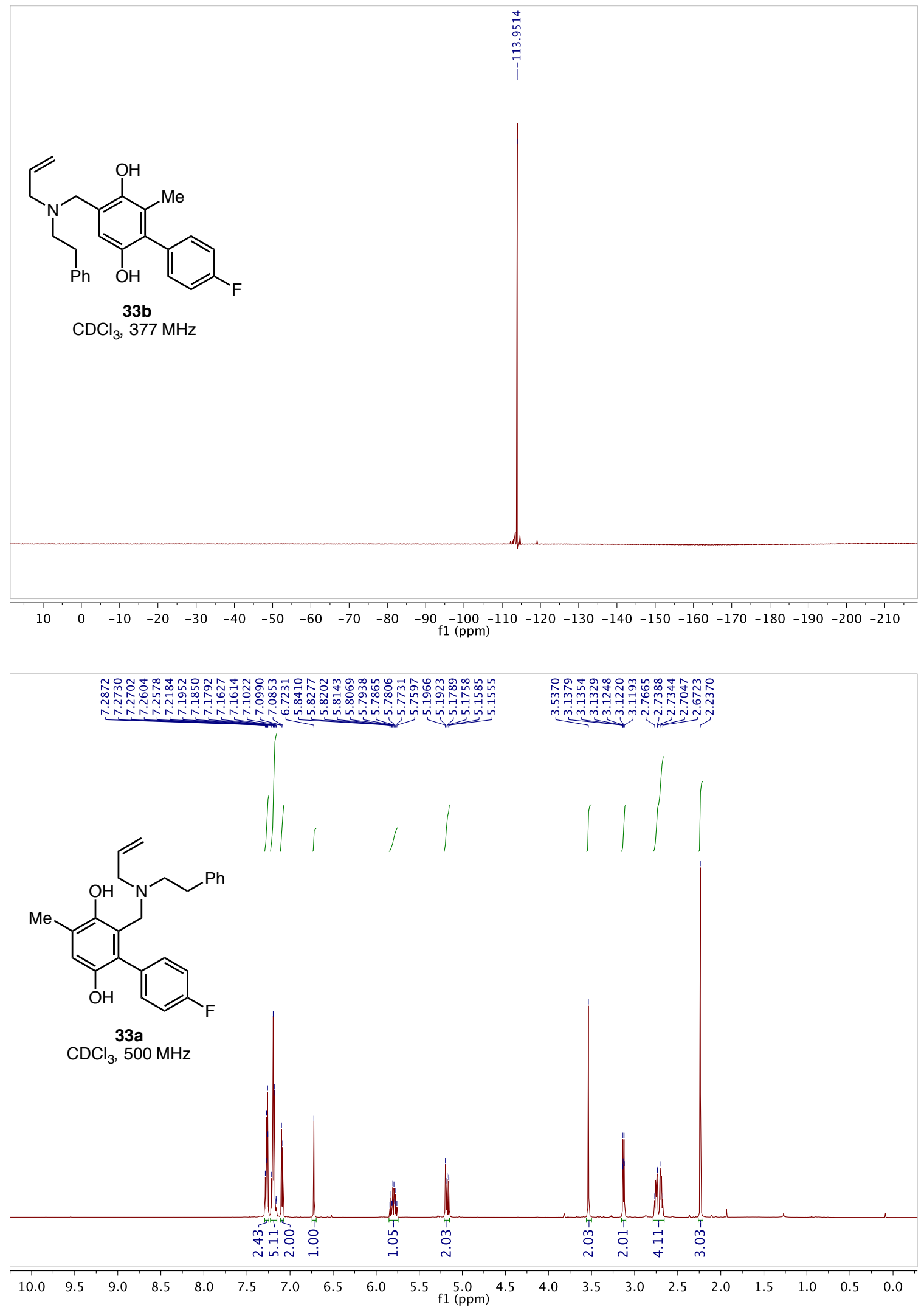



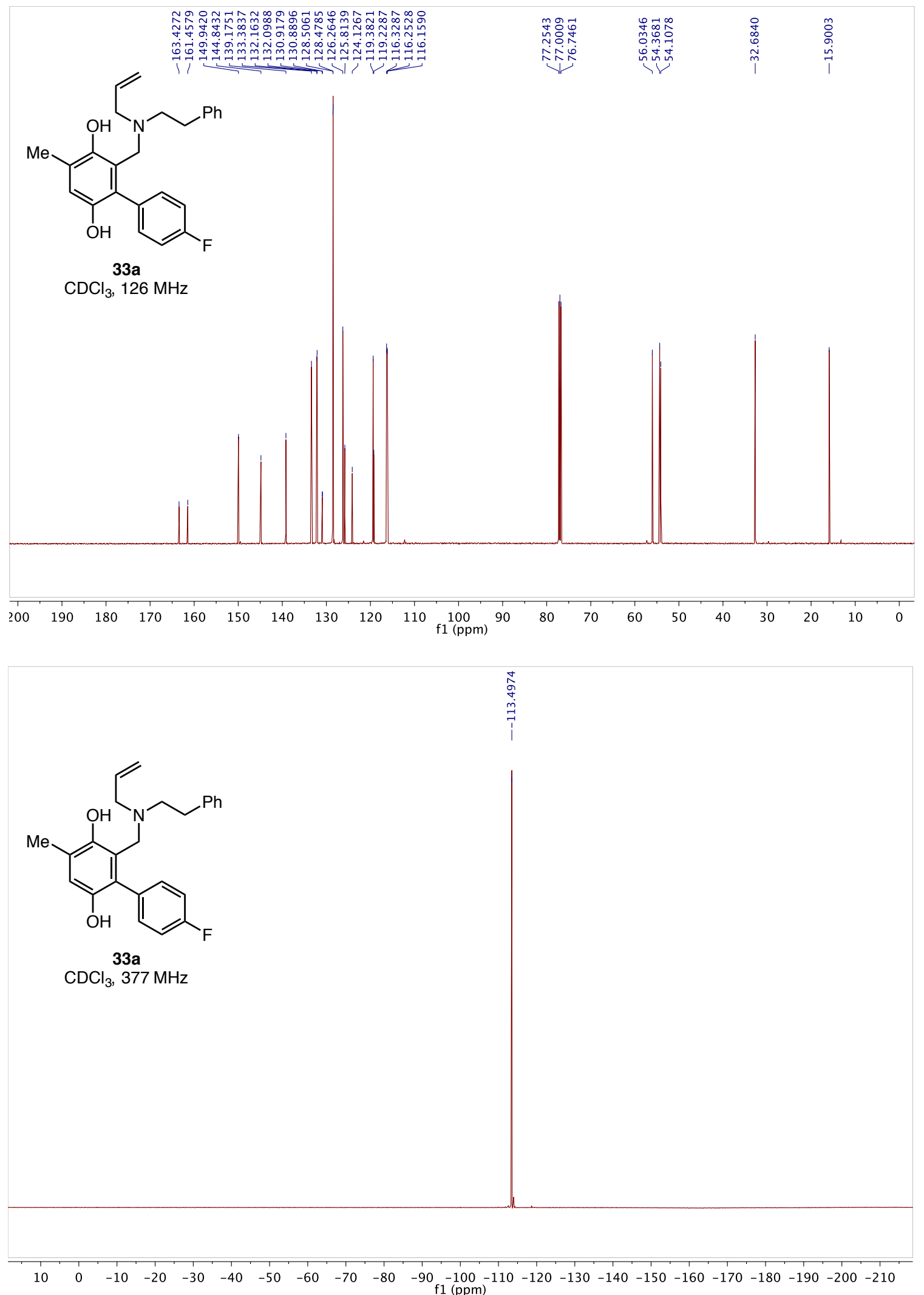

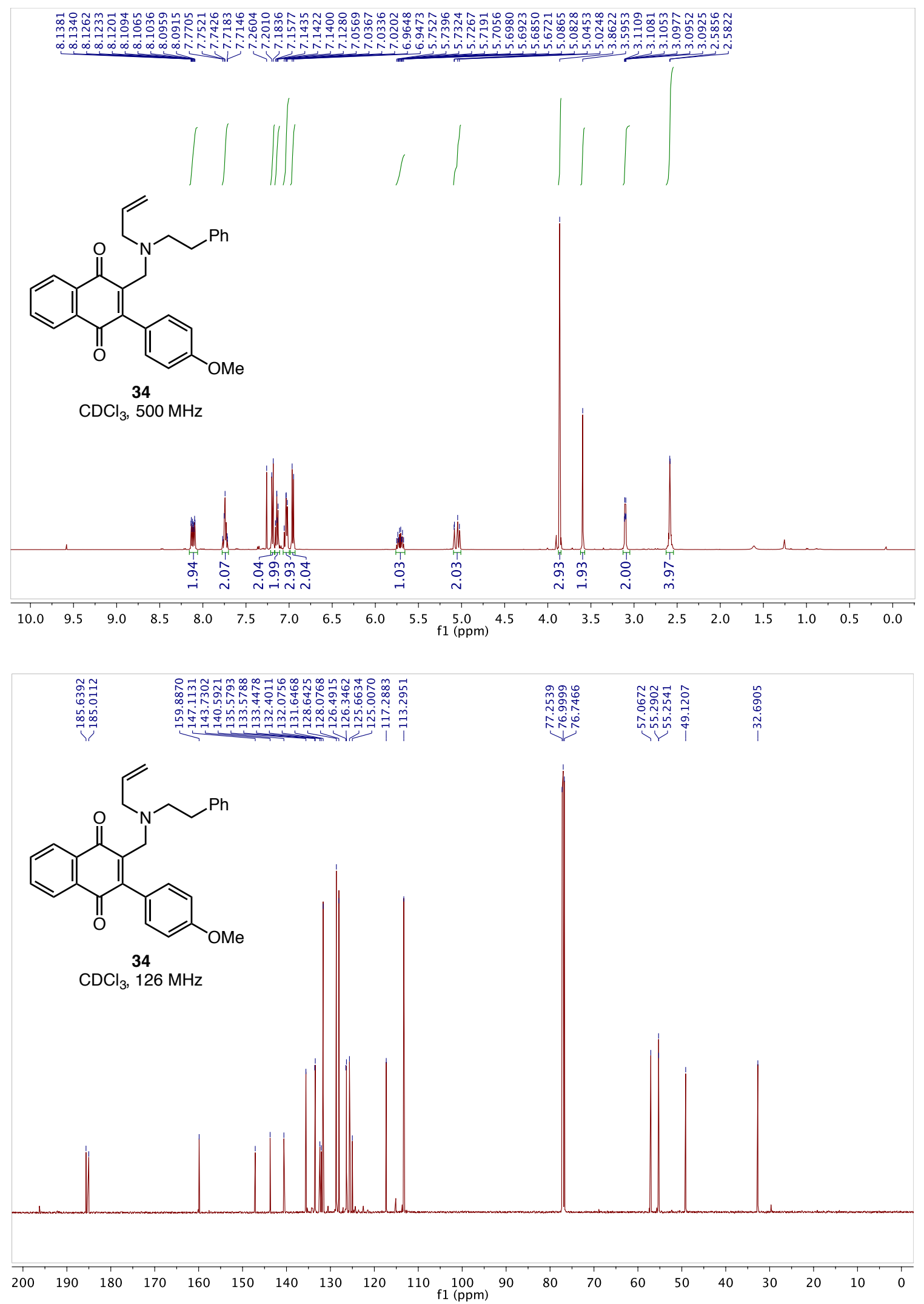


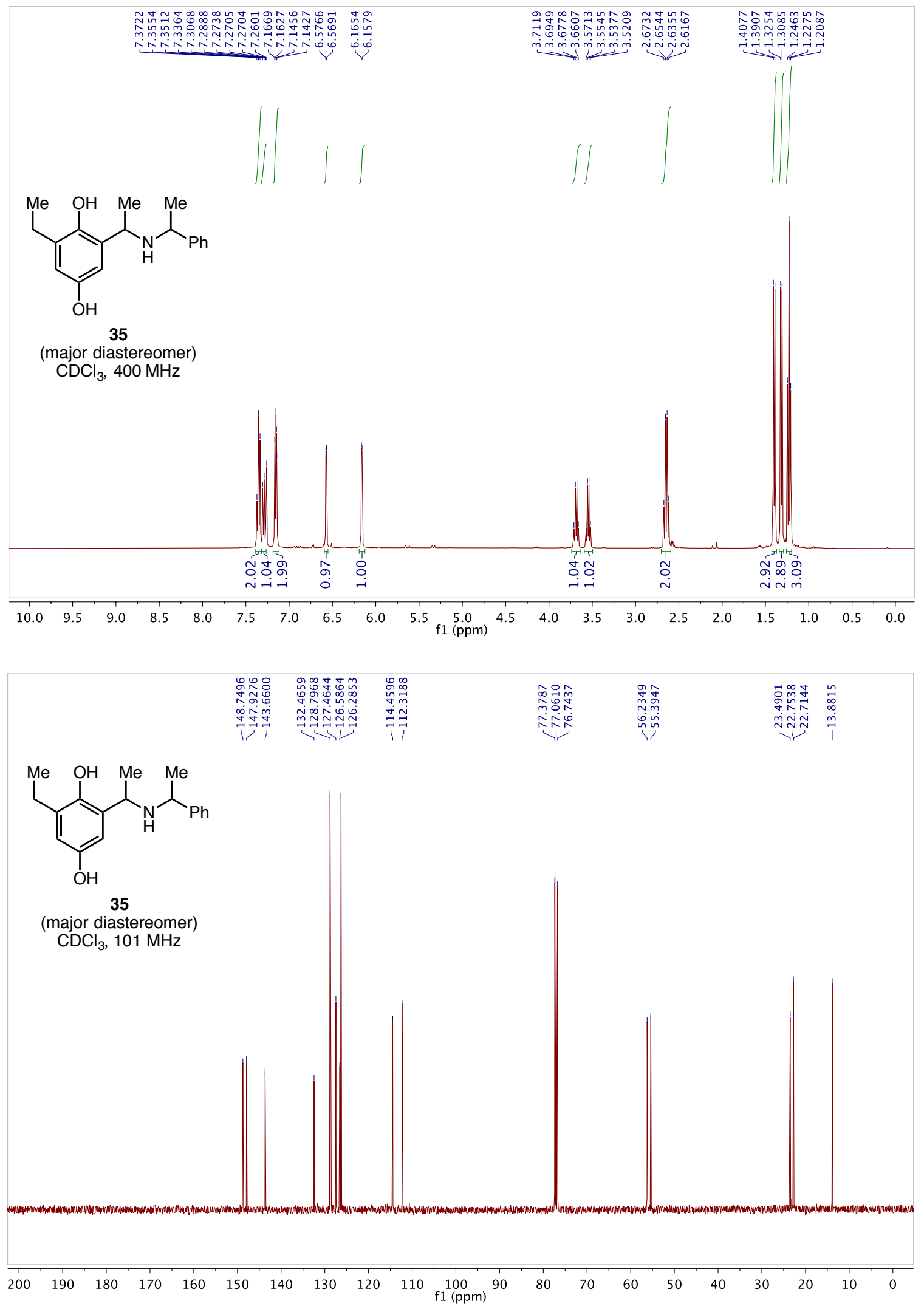




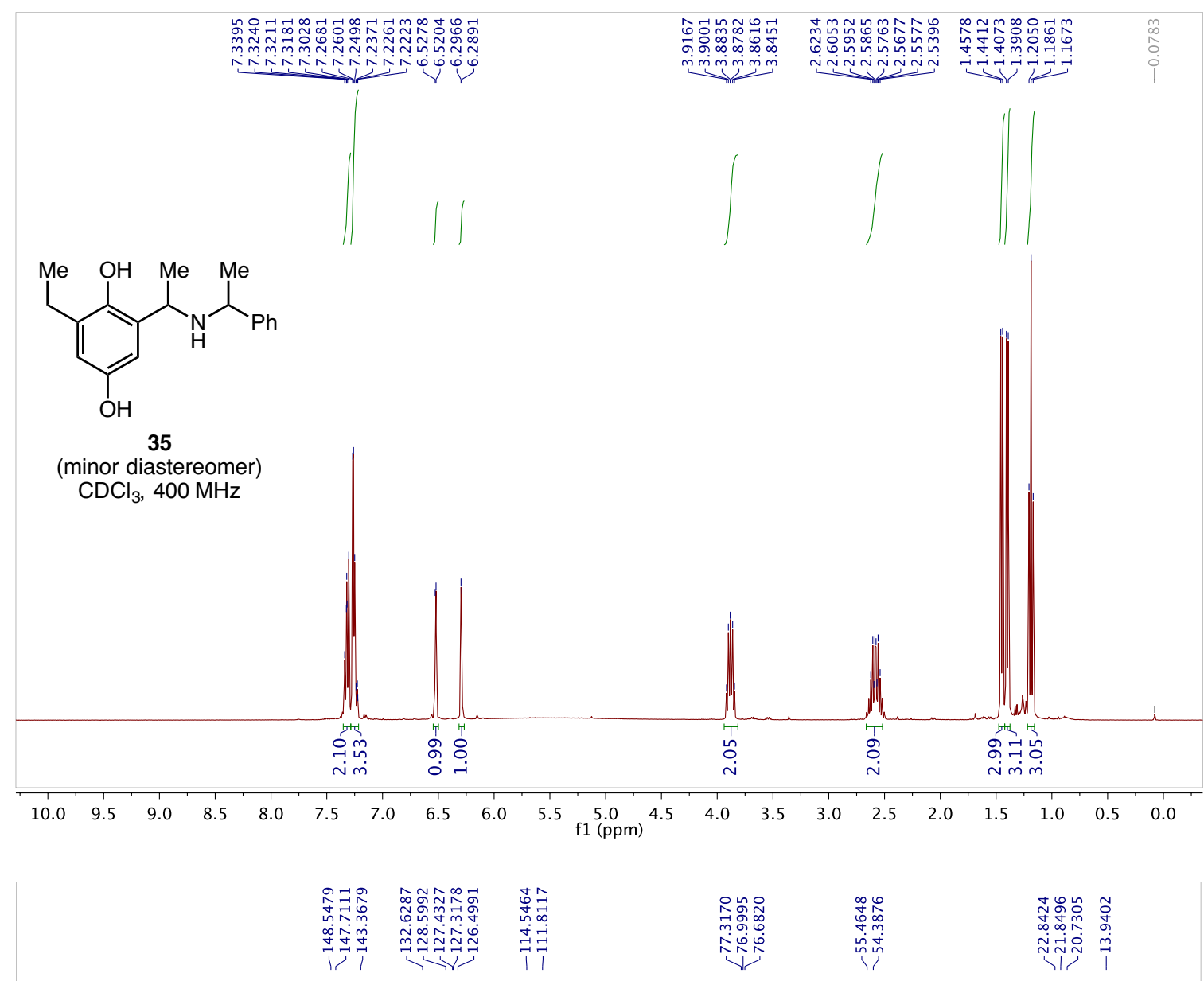<smiles>[Y6]C(NC(N)(c1ccccc1)c1cc(O)cc(C)c1O)c1ccccc1</smiles>

35

(minor diastereomer)

$\mathrm{CDCl}_{3}, 101 \mathrm{MHz}$

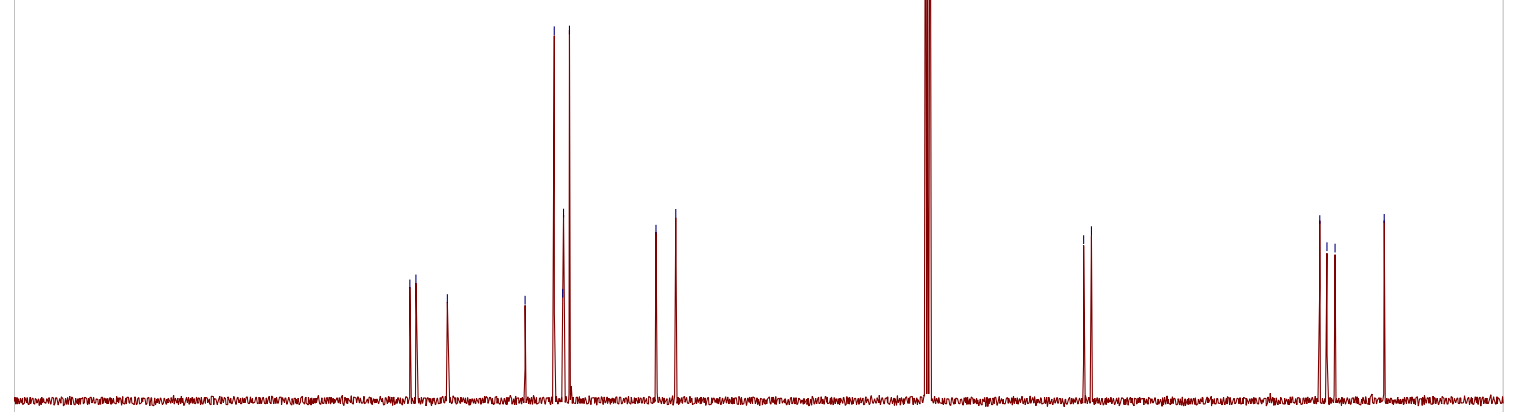

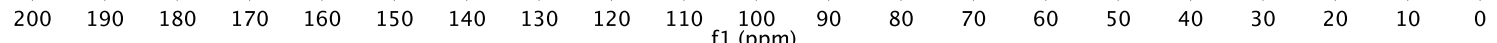



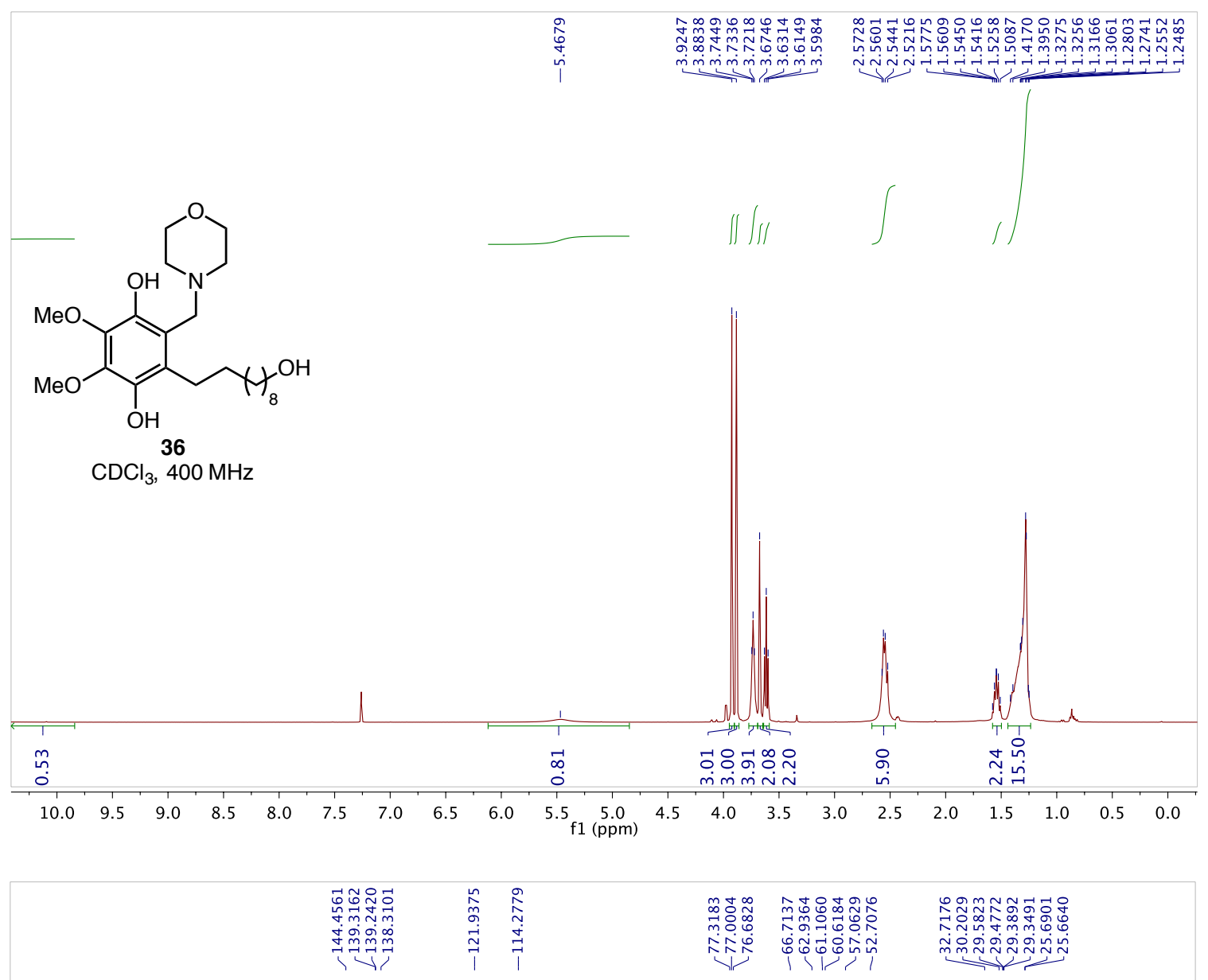<smiles>COc1c(O)c(CCC(=O)O)c(CN2CCOCC2)c(O)c1OC</smiles>

36

$\mathrm{CDCl}_{3}, 101 \mathrm{MHz}$

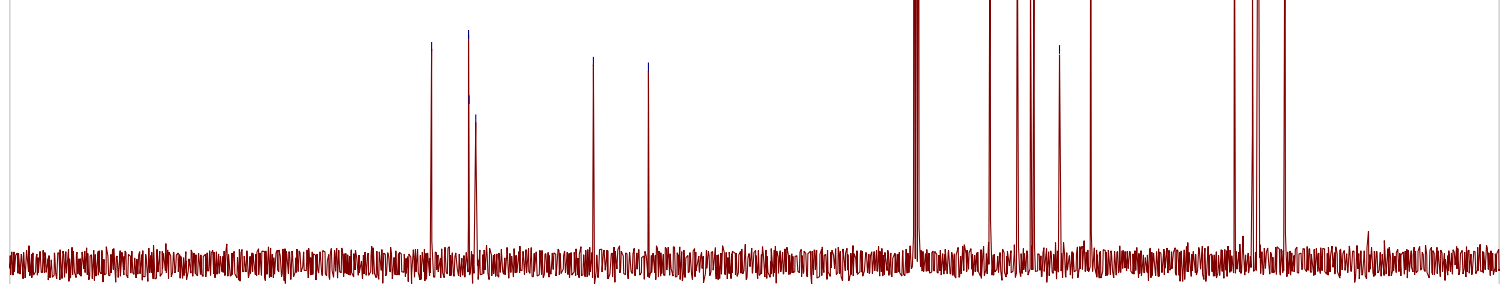

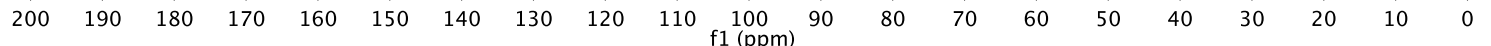



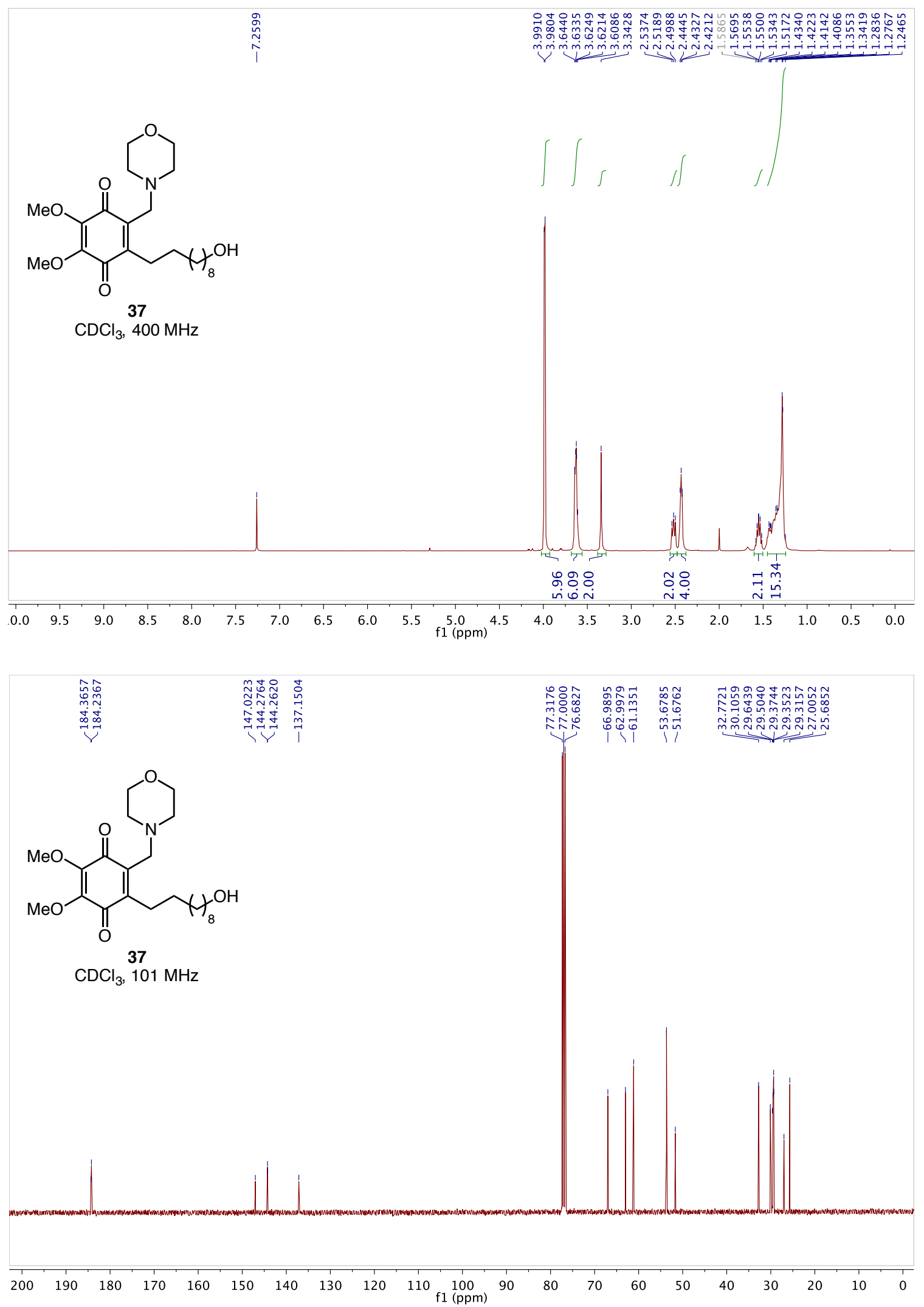